\title{
Year-long, broad-band, microwave backscatter observations of an alpine meadow over the Tibetan Plateau with a ground-based scatterometer
}

\author{
Jan G. Hofste ${ }^{1}$, Rogier van der Velde ${ }^{1}$, Jun Wen ${ }^{2}$, Xin Wang ${ }^{3}$, Zuoliang Wang ${ }^{3}$, Donghai Zheng ${ }^{4}$, \\ Christiaan van der $\mathrm{Tol}^{1}$, and Zhongbo $\mathrm{Su}^{1}$ \\ ${ }^{1}$ Faculty of Geo-Information Science and Earth Observation (ITC), \\ University of Twente, Enschede, the Netherlands \\ ${ }^{2}$ College of Atmospheric Sciences, Plateau Atmosphere and Environment Key Laboratory of Sichuan Province, \\ Chengdu University of Information Technology, Chengdu, China \\ ${ }^{3}$ Key laboratory of Land Surface Process and Climate Change in Cold and Arid Regions, Northwest Institute \\ of Eco-Environment and Resources, Chinese Academy of Sciences, Lanzhou, China \\ ${ }^{4}$ National Tibetan Plateau Data Center, Institute of Tibetan Plateau Research, \\ Chinese Academy of Sciences, Beijing, China \\ Correspondence: Jan G. Hofste (j.g.hofste@utwente.nl)
}

Received: 19 February 2020 - Discussion started: 11 March 2020

Revised: 19 April 2021 - Accepted: 12 May 2021 - Published: 16 June 2021

Abstract. A ground-based scatterometer was installed on an alpine meadow over the Tibetan Plateau to study the soil moisture and temperature dynamics of the top soil layer and air-soil interface during the period August 2017-August 2018. The deployed system measured the amplitude and phase of the ground surface radar return at hourly and half-hourly intervals over $1-10 \mathrm{GHz}$ in the four linear polarization combinations (vv, hh, hv, vh). In this paper we describe the developed scatterometer system, gathered datasets, retrieval method for the backscattering coefficient $\left(\sigma^{0}\right)$, and results of $\sigma^{0}$.

The system was installed on a $5 \mathrm{~m}$ high tower and designed using only commercially available components: a vector network analyser (VNA), four coaxial cables, and two dual-polarization broad-band gain horn antennas at a fixed position and orientation. We provide a detailed description on how to retrieve the backscattering coefficients for all four linear polarization combinations $\sigma_{p q}^{0}$, where $p$ is the received and $q$ the transmitted polarization ( $\mathrm{v}$ or h), for this specific scatterometer design. To account for the particular effects caused by wide antenna radiation patterns $(G)$ at lower frequencies, $\sigma^{0}$ was calculated using the narrow-beam approximation combined with a mapping of the function $G^{2} / R^{4}$ over the ground surface. ( $R$ is the distance between antennas and the infinitesimal patches of ground surface.) This approach allowed for a proper derivation of footprint positions and areas, as well as incidence angle ranges. The frequency averaging technique was used to reduce the effects of fading on the $\sigma_{p q}^{0}$ uncertainty. Absolute calibration of the scatterometer was achieved with measurements of a rectangular metal plate and rotated dihedral metal reflectors as reference targets.

In the retrieved time series of $\sigma_{p q}^{0}$ for L-band (1.5-1.75 GHz), S-band (2.5-3.0 GHz), C-band (4.5-5.0 GHz), and $\mathrm{X}$-band $(9.0-10.0 \mathrm{GHz})$, we observed characteristic changes or features that can be attributed to seasonal or diurnal changes in the soil: for example a fully frozen top soil, diurnal freeze-thaw changes in the top soil, emerging vegetation in spring, and drying of soil. Our preliminary analysis of the collected $\sigma_{p q}^{0}$ time-series dataset demonstrates that it contains valuable information on water and energy exchange directly below the airsoil interface - information which is difficult to quantify, at that particular position, with in situ measurement techniques alone.

Availability of backscattering data for multiple frequency bands (raw radar return and retrieved $\sigma_{p q}^{0}$ ) allows for studying scattering effects at different depths within the soil and vegetation canopy during the spring and 
summer periods. Hence further investigation of this scatterometer dataset provides an opportunity to gain new insights in hydrometeorological processes, such as freezing and thawing, and how these can be monitored with multi-frequency scatterometer observations. The dataset is available via https://doi.org/10.17026/dans-zfb-qegy (Hofste et al., 2021). Software code for processing the data and retrieving $\sigma_{p q}^{0}$ via the method presented in this paper can be found under https://doi.org/10.17026/dans-xyf-fmkk (Hofste, 2021).

\section{Introduction}

To comprehend the climate of the Tibetan Plateau, also known as the "Third Pole Environment", the transfer processes of energy and water at the land-atmosphere interface must be understood (Seneviratne et al., 2010; Su et al., 2013). Main states of interest are the dynamics of soil moisture and temperature (Zheng et al., 2017a). Together with sensors embedded into the deeper soil layers, microwave remote sensing is suitable to study these dynamics since it directly probes the top soil layer within the antenna footprint.

A ground-based microwave observatory was installed on an alpine meadow over the Tibetan Plateau, near the town of Maqu. The observatory consists of a microwave radiometer system called ELBARA-III (ETH L-Band radiometer for soil moisture research) (Schwank et al., 2010; Zheng et al., $2017 \mathrm{~b}$ ) and an microwave scatterometer. Both continuously measure the surface's microwave signatures with a temporal frequency of once every hour. The ELBARA-III was installed in January 2016 and is currently still measuring (Zheng et al., 2019; Su et al., 2020); the scatterometer was installed in August 2017 and continued to operate until July 2019.

This paper describes the scatterometer system and the collected dataset over the period August 2017-August 2018 (Hofste et al., 2021). The radar return amplitude and phase were measured over a broad $1-10 \mathrm{GHz}$ frequency band at all four linear polarization combinations (vv, hv, vh, hh). The scatterometer measured the radar return over a prolonged period with its antennas in a fixed orientation, resulting in frequency-dependent incidence angle ranges varying from of $0^{\circ} \leq \theta \leq 60^{\circ}$ for L-band $(1.625 \mathrm{GHz})$ to $47^{\circ} \leq \theta \leq 59^{\circ}$ for X-band $(9.5 \mathrm{GHz})$. During the summers of 2017 and 2018 additional experiments were conducted to assess the angular dependence of the backscatter and homogeneity of the local ground surface.

Many other studies exist employing ground-based systems to study microwave backscatter from land. Rather than an airborne or spaceborne system, ground-based systems allow for high temporal coverage and a high degree of control over the experimental circumstances. Geldsetzer et al. (2007) and Nandan et al. (2016) used specially developed radar systems by ProSensing Inc. to study backscattering from sea ice in the period 2004-2011: one system for C-band and another for $\mathrm{X}$ - and Ku-band. Details on a similar S-band system can be found in Baldi (2014). The SnowScat system, developed by
Gamma Remote Sensing AG (Werner et al., 2010), is another scatterometer that operates over 9-18 GHz and measures the full polarimetric backscatter autonomously over many elevation and azimuth angles. Lin et al. (2016) used it during multiple winter campaigns in the 2009-2012 period at two different locations to study the scattering properties of snow layers. Like in this study, others also designed their scatterometer architecture around a commercially available vector network analyser (VNA). For instance, Joseph et al. (2010) used data measured by a truck-based system, operating at C- and L-band, in summer 2002 to study the influence of corn on the retrieval of soil moisture from microwave backscattering. For every band they placed one antenna to transmit and receive on top of a boom. Selection of the individual polarization channels was realized using radio-frequency switches. Similar is the University of Florida L-band Automated Radar System (UF-LARS) (Nagarajan et al., 2014), used by for example Liu et al. (2016), to measure soil moisture at L-band from a Genie platform during summer 2012. Another example is the Hongik Polarimetric Scatterometer (HPS) (Hwang et al., 2011), with which microwave backscatter from bean and corn fields was measured in 2010 and 2013 respectively (Kweon and Oh, 2015). Similar to our study, Kim et al. (2014) used a scatterometer with its antenna in a fixed position and orientation to measure the backscattering during all growth stages of winter wheat at L-, C-, and X-band during 2011-2012.

The temporal resolution and measurement period covered by the scatterometer dataset reported in this paper permits studying both seasonal and diurnal dynamics of microwave backscattering from an alpine meadow ecosystem. This in turn allows for investigating the local soil moisture dynamics, the freeze-thaw process, and growth/decay stages of vegetation. Because of the broad frequency range measured (1$10 \mathrm{GHz}$ ), wavelength-dependent effects of surface roughness and vegetation scattering can be studied as well.

This paper is organized as follows. First the study area is described. Next, details are provided on the instrumentation used, measurements performed, and method for retrieving the backscattering coefficient $\sigma^{0}\left(\mathrm{~m}^{2} \mathrm{~m}^{-2}\right)$. We then present an overview of the retrieved $\sigma^{0}$ time-series dataset and show how $\sigma^{0}$ varies across seasons and on a diurnal timescale. In the discussion section the angular and spatial variability of $\sigma^{0}$ at the study area and measurement uncertainty are described. Technical details on all aspects of the scatterometer measurements and $\sigma^{0}$ calculation are included in the Ap- 
pendix. A list of symbols can be found at the end of this paper.

\section{Study region and climate}

In August 2017 the scatterometer was installed on the tower of the Maqu measurement site (Maqu site) (Zheng et al., 2017b) and operated over the period August 2017-June 2019. The Maqu site is situated in an alpine meadow ecosystem (Miller, 2005) on the Tibetan Plateau, Fig. 1a. The site's coordinates are $33^{\circ} 55^{\prime} \mathrm{N}, 102^{\circ} 10^{\prime} \mathrm{E}$, at $3500 \mathrm{~m}$ elevation. The site is located close to the town Maqu of the Gansu province of China.

Besides the scatterometer, other remote sensing sensors placed on the tower are the ELBARA-III radiometer (Schwank et al., 2010) and the optical spectroradiometer system Piccolo (MacArthur et al., 2014), Fig.1b. The ELBARAIII system has been measuring L-band microwave emission since January 2016 to this date (Zheng et al., 2019; Su et al., 2020). The Piccolo system measured the reflectance and suninduced chlorophyll fluorescence of the vegetation over the period July-November 2018.

According to Peel et al. (2007) the climate at Maqu is characterized by the Köppen-Geiger classification as "Dwb": cold with dry winters. Winter (December-February) and spring (March-May) are cold and dry, while the summer (June-August) and autumn (August-November) are mild with monsoon rain.

The ecosystem classification of the Maqu site is "alpine meadow" according to Miller (2005). The vegetation around the Maqu site consists of grasses for the most part. The growing season starts at the end of April and ends in October, when above-ground biomass turns brown and loses its water. During the growing season the meadows are regularly grazed by livestock. To prevent the livestock from entering the site and damaging the equipment, a fence is placed around the Maqu site. As a result there is no grazing within the site, causing the vegetation to be more dense and higher than that of the surroundings. Also a layer of dead plant material from the previous year remains present below the newly emerged vegetation. In Appendix Sect. A1 some photographs are shown of the Maqu site during different seasons, which provide an impression of the site's phenology.

\section{Methodology}

\subsection{Supporting measurements}

Together with the scatterometer, measurements following hydrometeorological quantities were recorded over the period August 2017-August 2018: depth profile of volumetric soil moisture $m_{\mathrm{v}}\left(\mathrm{m}^{3} \mathrm{~m}^{-3}\right)$ and soil temperature $T_{\text {soil }}\left({ }^{\circ} \mathrm{C}\right)$, air temperature $T_{\text {air }}\left({ }^{\circ} \mathrm{C}\right)$, precipitation $(\mathrm{mm})$, and the short- and long-wave up- and downward irradiance $\left(\mathrm{W} \mathrm{m}^{-2}\right)$. Details on used sensors can be found in Appendix Sect. A2.
The depth profile of $m_{\mathrm{v}}\left(\mathrm{m}^{3} \mathrm{~m}^{-3}\right)$ was measured with an array of 20 capacitance sensors, type 5TM (manufacturer: Meter Group), that were installed at depths ranging from $2.5 \mathrm{~cm}$ to $1 \mathrm{~m}$ ( $\mathrm{Lv}$ et al., 2018). All sensors in the array are also equipped with a thermistor, enabling the measurement of $T_{\text {soil }}\left({ }^{\circ} \mathrm{C}\right)$. The soil moisture and temperature was logged every $15 \mathrm{~min}$ for the period of August 2017-August 2018 with Em50 data loggers (manufacturer: Meter Group) that were buried near the sensors. The location of the buried sensor array is indicated in Fig. 2. Results of these hydrometeorological measurements over the period August 2017-August 2018 can be found in Appendix Sect. A2 as well. With a handheld impedance probe, type ThetaProbe ML2x (manufacturer: Delta-T Devices), the spatial variability of $m_{\mathrm{v}}$ in the top $2.5-5 \mathrm{~cm}$ soil layer over the Maqu site was measured (Appendix Sect. A3).

To quantify the vegetation cover at the Maqu site, measurements were performed on $2 \mathrm{~d}$ during the 2018 summer, namely 12 July and 17 August. Vegetation height, aboveground biomass (fresh and oven-dried), and leaf area index (LAI) $\left(\mathrm{m}^{2} \mathrm{~m}^{-2}\right)$ were measured at ten $1.2 \times 1.2 \mathrm{~m}^{2}$ sites around the periphery of the "no-step zone" indicated in Fig. 2. The vegetation height of a single site was determined as the maximum value of the histogram obtained by taking $\geq 30$ readings with a thin ruler at random points within the site area. For each site, above-ground biomass and LAI were determined from harvested vegetation within one or two disk areas defined by a $45 \mathrm{~cm}$ diameter ring. Immediately after harvest all biomass was placed in airtight bags so that the fresh and dry biomass could be determined by weighing the bag's content before and after drying in an oven. The LAI was determined immediately after harvest with part of the harvested fresh biomass by the method described in He et al. (2007). The obtained average quantities over the 10 sites are summarized in Appendix Sect. A4.

\subsection{Scatterometer}

\subsubsection{Instrumentation}

The main components of the scatterometer are a two-port vector network analyser (VNA), type PNA-L 5232A (manufacturer: Keysight); four $3 \mathrm{~m}$ long phase-stable coax cables, type Sucoflex SF104PEA (manufacturer: Huber + Suhner); and two dual-polarized broad-band horn antennas, type BBHX9120LF (manufacturer: Schwarzbeck); see Fig. B1. The antenna radiation patterns are measured in the principal planes by the manufacturer over the $1-10 \mathrm{GHz}$ band (Schwarzbeck Mess-Elektronic OHG, 2017). As a summary, the full width at half maximum (FWHM) intensity beamwidths over frequency are shown in Fig. B3. To protect the VNA from weather it is placed inside a waterproof enclosure equipped with fans to provide air ventilation.

Deployed reference targets to calibrate the scatterometer were a rectangular plate and two dihedral reflectors. The 


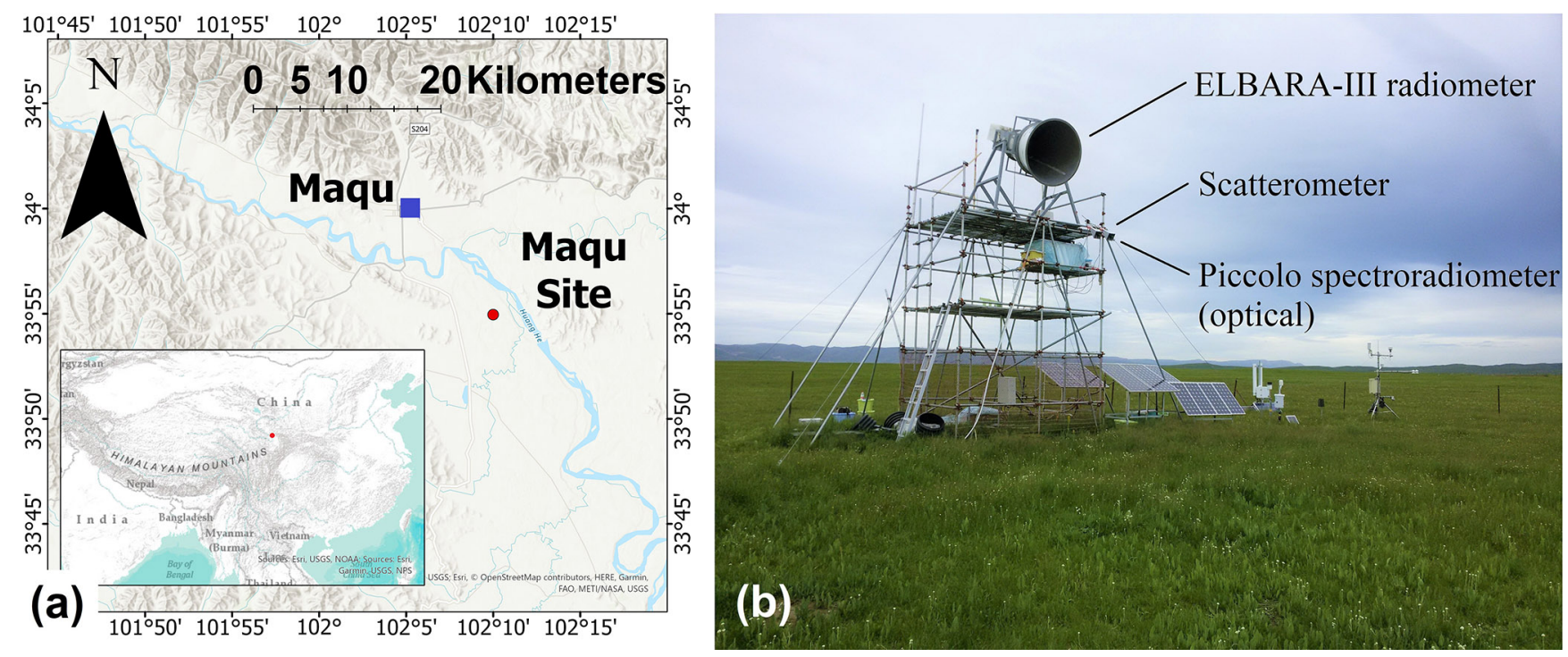

Figure 1. (a) Location of Maqu measurement site on eastern part of the Tibetan Plateau. (b) Tower of Maqu site containing the scatterometer, the ELBARA-III radiometer, and Piccolo optical spectroradiometer.

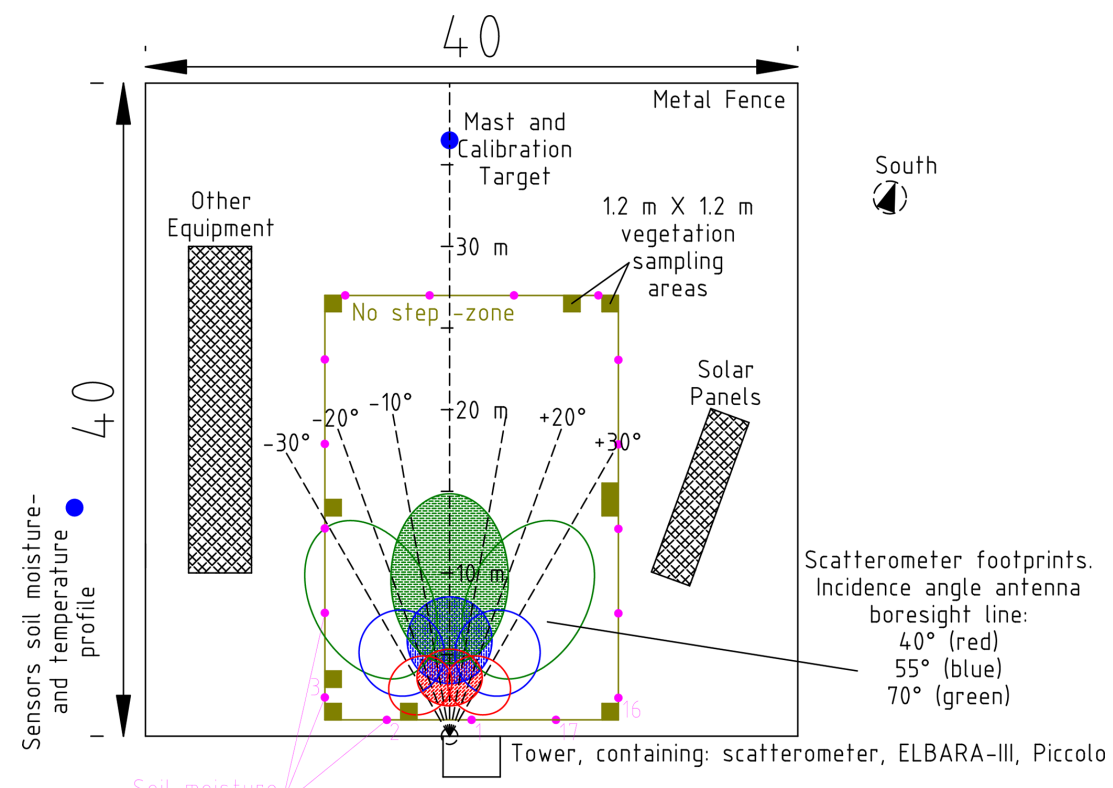

Figure 2. Map of the Maqu site. Scatterometer footprints for C-band with vv polarization are shown for different $\alpha_{0}\left(40,55,70^{\circ}\right)$ and $\phi$ $\left(-30,-20, \ldots, 30^{\circ}\right)$ angles. For time-series measurement antennas were fixed at $\alpha^{0}=55^{\circ}$ and $\phi=0^{\circ}$.

rectangular plate reflector was constructed from lightweight foam board covered with $100 \mu \mathrm{m}$ aluminium foil and had frontal dimensions $a=85 \mathrm{~cm} \times b=65 \mathrm{~cm}$. A small dihedral reflector was constructed from steel, and its frontal dimensions were $a=57 \mathrm{~cm} \times b=38 \mathrm{~cm}$. A second large dihedral reflector was also constructed with foam board and aluminium foil, and its frontal dimensions were $a=$ $120 \mathrm{~cm} \times b=65 \mathrm{~cm}$. A height-adjustable metal mast was used to position the reference targets. To minimize reflec- tion from this mast, it was covered by pyramidal absorbers, type 3640-300 (manufacturer: Holland Shielding), having a $35 \mathrm{~dB}$ reflection loss for normal incidence at $1 \mathrm{GHz}$.

\subsubsection{Experimental setup and procedures}

The scatterometer is placed on a tower as shown in Fig. 1b. The two antenna apertures are at a distance approximately $H_{\text {ant }}=5 \mathrm{~m}$ above the ground $\left(H_{\text {ant }}\right.$ depends on the an- 
tenna boresight angle $\alpha_{0}$ ) and are separated from each other horizontally by $W_{\text {ant }}=0.4 \mathrm{~m}$. The connection scheme of the VNA and the two antennas is described in Appendix Sect. B1. In Appendix Sect. B2 further details on the setup geometries can be found. During all experiments, VNA measurements were performed with a stepped $0.75-10.25 \mathrm{GHz}$ frequency sweep at $3 \mathrm{MHz}$ resolution (3201 points). The dwell time per measured frequency was $1 \mu \mathrm{s}$, which is equivalent to a two-way travelling distance for the microwave signal of $150 \mathrm{~m}$. The intermediate-frequency (IF) bandwidth was minimized to $1 \mathrm{KHz}$ to increase the signal-to-noise ratio.

The radar return from the rectangular metal plate reference target was used to calibrate the scatterometer for the copolarization channels. The two metal dihedral reflectors were used as depolarizing reference targets (Nesti and Hohmann, 1990) to calibrate the cross-polarization channels. We used two dihedrals, measured at different distances $R_{0}(\mathrm{~m})$, in order to meet requirements concerning target size, target distance (plane wave criteria), and ground-to-target interference removal. Readers are referred to Appendix Sect. B3 for the measurement details and validation-exercise results.

Time-domain filtering, or gating, was used as part of postprocessing to remove the antenna-to-antenna coupling and undesired scattering contributions from the radar return signal for both the reference target and the ground return measurements. The application of gating with VNA-based scatterometers is described in more detail in for example Jersak et al. (1992) or De Porrata-Dória i Yagüe et al. (1998). Details on our gating process and related peculiarities regarding our scatterometer can be found in Appendix Sect. B4.

In this paper, we focus on the time-series measurements of $\sigma^{0}$ over a 1-year period, during which measurements were taken either once or twice per hour. With this experiment, the antennas were fixed on a tower rod, such that the angle between the antenna boresight line and the ground surface normal $\alpha_{0}$ was $55^{\circ}$ and the azimuth angle $\phi$ was fixed at $0^{\circ}$ as shown in Fig. 2. Although varying the antennae orientations (using automatic motorized rotational stages) to measure backscatter under various incidence and azimuth angles would be preferable from an experimental perspective, this approach was abandoned because it would make the setup extra vulnerable to system failures. Measurements of $\sigma^{0}$ for different $\alpha_{0}$ and $\phi$ angles at the Maqu site were, however, performed during 3 separate days. These measurements are discussed in Sect. 5.3. Before installing the scatterometer at the Maqu site, exploratory experiments were performed in which $\sigma^{0}$ over $\alpha_{0}$ was measured for asphalt and subsequently compared to results in other studies (Sect. 5.1). Table 1 summarizes all experiment geometries and dates of execution. For the angular-variation experiments the scatterometer antennas were mounted on a motorized rotational stage. Depending on the angle $\alpha_{0}, H_{\text {ant }}$ would vary according to $H_{\text {ant }}=H_{0}-0.5 \cos \left(\alpha_{0}\right)$, with $H_{0}=2.95$ or $5.2 \mathrm{~m}$ for the asphalt or Maqu experiments respectively. All angularvariation experiments were conducted within one afternoon.

\subsection{3 $\sigma^{0}$ retrieval procedure}

The power received by a monostatic radar or scatterometer system from a distributed target with backscattering coefficient $\sigma_{p q}^{0}(\theta)\left(\mathrm{m}^{2} \mathrm{~m}^{-2}\right)$ is given by the radar equation (Ulaby et al., 1982)

$P_{p}^{\mathrm{RX}}=\frac{\lambda^{2}}{64 \pi^{3}} P_{q}^{\mathrm{TX}} G_{0}^{2} \int \frac{G^{2}}{R^{4}} \sigma_{p q}^{0}(\theta) \cdot \mathrm{d} A$,

where it is assumed that the same antenna is used for both transmitting (TX) and receiving $(\mathrm{RX}) . P_{q}^{\mathrm{TX}}$ is the transmitted and $P_{p}^{\mathrm{RX}}$ the received power respectively $(\mathrm{W})$. The subscripts of the powers refer to the linear polarization directions: horizontal (h) or vertical (v). With $\sigma_{p q}^{0}$ the first subscript refers to the polarization direction of the scattered and the second to that of the incident wave. $G(-)$ denotes the normalized angular gain pattern of the antenna with peak value $G_{0}(-)$. Equation (1) represents an ideal lossless system - in practice any scatterometer has frequency-dependent losses or other signal distortions. These frequency-dependent phase and amplitude modulations can be accounted for by measuring the radar return of a reference target $P_{p}^{0}$ with known radar cross section (RCS) $\sigma_{p q}\left(\mathrm{~m}^{2}\right)(\mathrm{Eq}$. B2) to calibrate the system. This procedure, often referred to as external calibration, is mathematically represented by

$P_{p}^{\mathrm{RX}}=P_{p}^{0} \frac{\left(R_{0}\right)^{4}}{\sigma_{p q}} \int \frac{G^{2}}{R^{4}} \sigma_{p q}^{0}(\theta) \cdot \mathrm{d} A$,

where $R_{0}(\mathrm{~m})$ is the distance at which the reference target was measured. In the case of a scatterometer with narrow beamwidth antenna, all integrand terms of Eq. (2) can be approximated as being constants, the so-called "narrow-beam approximation" (Wang and Gogineni, 1991), so that we obtain

$P_{p}^{\mathrm{RX}}=P_{p}^{c} \frac{\left(R_{0}\right)^{4}}{\sigma_{p q}} \frac{1}{\left(R_{\mathrm{fp}}\right)^{4}} \sigma_{p q}^{0}(\theta) A_{\mathrm{fp}}$,

where $A_{\mathrm{fp}}$ is the scatterometers "footprint", notably the area $\left(\mathrm{m}^{2}\right)$ for which the surface projected antenna beam intensity is equal to or larger than half its maximum value. $R_{\mathrm{fp}}(\mathrm{m})$ refers to the distance between the antenna and footprint centre.

For this dataset $\sigma_{p q}^{0}(\theta)$ is estimated by employing Eq. (3) in combination with a mapping of the term $G^{2} / R^{4}(x, y)$ from Eq. (2) over the ground surface. Due to the wide antenna radiation patterns, especially with low frequencies, the area that is to be associated with the measured scatterometer signal, i.e. the footprint, is typically not located where the antenna boresight line intersects the ground surface. Instead the footprint appears closer to the tower base. Figure 3 demonstrates this effect for the case of $5 \mathrm{GHz}$ at $\alpha_{0}=55^{\circ}$, by showing the mapping of $G^{2} / R^{4}$ over the ground surface. This footprintshift effect is strongest with the widest antenna radiation patterns (thus with low frequencies) and for large $\alpha_{0}$ angles. The 
Table 1. Overview of performed scatterometer experiments and their respective $\alpha_{0}$ and $\phi$ ranges. Antennae aperture height $H_{\text {ant }}$ depends on $\alpha_{0}$.

\begin{tabular}{lllll}
\hline & Date & $\phi\left(^{\circ}\right)$ & $\alpha_{0}\left({ }^{\circ}\right)$ & $H_{\text {ant }}(\mathrm{m})$ \\
\hline Angular variation $\sigma_{0}$ asphalt & 4 May 2017 & 00 & $35,40, \ldots, 75$ & $2.55,2.55, \ldots, 2.80$ \\
\hline Angular variation $\sigma_{0}$ Maqu & 25 August 2017 & $\begin{array}{l}-20,-15,-10,-05,00,+10, \\
+15,+20\end{array}$ & $35,40, \ldots, 70$ & $4.80,4.80, \ldots, 5.05$ \\
\hline Angular variation $\sigma_{0}$ Maqu & 29 June 2018 & $\begin{array}{l}-30,-20,-15,-10,-05,00, \\
+05,+10,+20,+25,+30\end{array}$ & $35,40, \ldots, 70$ & $4.80,4.80, \ldots, 5.05$ \\
\hline Angular variation $\sigma_{0}$ Maqu & 19 August 2018 & $\begin{array}{l}-30,-20,-10,00,+10,+20, \\
+30,\end{array}$ & $35,55,70$ & $4.80,4.90,5.05$ \\
\hline Time series $\sigma_{0}$ Maqu & 26 August 2017- & 00 & & \\
& 26 August 2018 & & & 4.70 \\
\hline
\end{tabular}

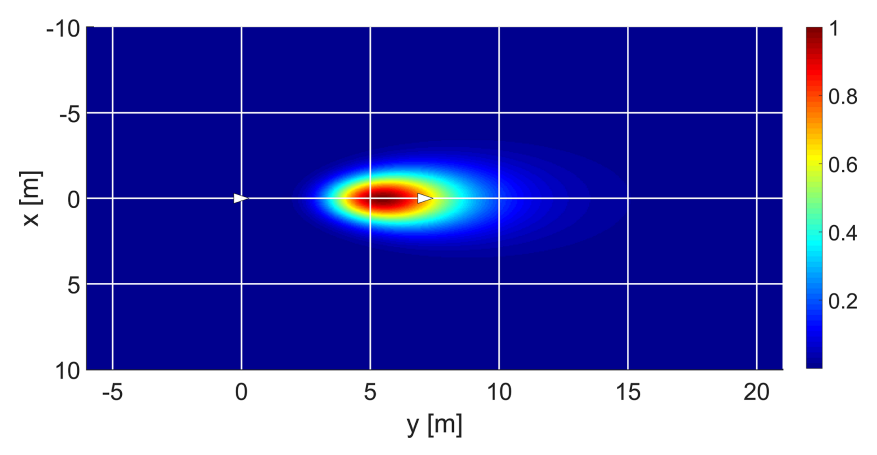

Figure 3. Example of $G^{2} / R^{4}(x, y)$ with Gaussian antenna radiation patterns. Plot normalized to its peak value. $x$ and $y$ are ground surface coordinates. The white triangle at coordinate $(0,0)$ represents the tower location and the other white triangle indicates the intersection point of the antenna boresight line and the ground surface. $\alpha_{0}=55^{\circ}, f=5 \mathrm{GHz}$ and polarization is vv.

footprint position and dimensions were found using the mapping $G^{2} / R^{4}(x, y)$ over the ground surface. The applied criterion was that the footprint contains $50 \%$ of the total projected intensity onto the ground surface. After the footprint edges were defined the incidence angle ranges were derived from them using trigonometry.

Because of the low directivity (gain) of the antennas and unknown nature of $\sigma_{p q}^{0}$ over $\theta$, there is an inherent uncertainty in our retrieved $\sigma_{p q}^{0}$ values (for a certain $\theta$ range). This matter is discussed further in Sect. 5.2.

In Fig. 4 the procedure for deriving the backscattering coefficient is depicted. The equations used therein are derived from Eq. (3). Refer to Appendix Sect. C1 for more information. The different steps indicated in the figure are explained here.

1. We start with $E_{\mathrm{e}}\left(\mathrm{V} \mathrm{m}^{-1}\right)$, the measured backscattered electric field from the ground target incident on the receiving antenna. The subscript e denotes "envelope" magnitude of the complex signal, as in Ulaby et al.
$(1988)^{1}$. This quantity is measured over the full $0.75-$ $10.25 \mathrm{GHz}$ band at angle $\alpha_{0}: E_{\mathrm{e}}\left(f, \alpha_{0}\right)$. Bandwidths (BW) are selected based on the change in $G(\alpha, \beta)$ over frequency (Appendix Sect. B4), the number of independent frequency samples $N$ that may be retrieved from $\mathrm{BW}$, and the estimated change in backscattering properties over frequency of the ground surface as is discussed in Appendix Sect. C2. Result is the bandwidth selection $E_{\mathrm{e}}\left(\mathrm{BW}, \alpha_{0}\right)$.

2. With BW and $\alpha_{0}$ as input, $G^{2} / R^{4}(x, y)$ is mapped for all frequencies within $\mathrm{BW}$ using the antenna radiation patterns measured by the manufacturer. The region associated with $50 \%$ of the total projected intensity onto the ground is determined to set appropriate gating times, or distances $r_{\mathrm{sg}}$ and $r_{\mathrm{eg}}(\mathrm{m})$, and for calculating the $A_{\mathrm{fp}}$, $R_{\mathrm{fp}}$, and the $\theta$ range. Half the pulse width $c /(2 \mathrm{BW})$ is subtracted from $r_{\mathrm{sg}}$ and added to $r_{\mathrm{eg}}$, and quantities $A_{\mathrm{fp}}$, $R_{\mathrm{fp}}$, and the $\theta$ range are changed accordingly.

3. The gate is applied to $E_{\mathrm{e}}\left(\mathrm{BW}, \alpha_{0}\right)$, resulting in the gated backscattered field $E_{\mathrm{e}}^{\mathrm{g}}\left(\mathrm{BW}, \alpha_{0}\right)$, where the superscript $\mathrm{g}$ indicates that the signal is gated.

4. The bandwidth-average coupling remnant $\left\langle E_{\mathrm{cr}}^{\mathrm{g}}\right\rangle$ $\left(\mathrm{Vm}^{-1}\right)$ and minimal detectable signal $E_{\mathrm{b}}\left(\mathrm{Vm}^{-1}\right)$ are subtracted from $E_{\mathrm{e}}^{\mathrm{g}}\left(\mathrm{BW}, \alpha_{0}\right)$ for each measured frequency. $E_{\mathrm{cr}}^{\mathrm{g}}$ is an offset formed by part of the signal transmitted from the transmit antenna coupling directly into the receive antenna (antenna cross coupling). Although the majority of this coupling can be filtered out by using time-domain gate filtering, a remnant is still present (hence "coupling remnant" in the subscript) and must be accounted for (Appendix Sect. E4). Note that the same gate as with $E_{\mathrm{e}}^{\mathrm{g}}$ is applied. A similar form

\footnotetext{
${ }^{1}$ In reality the measured fields or signals remain complex until after the gating process. We, however, stick to this terminology for clarity.
} 


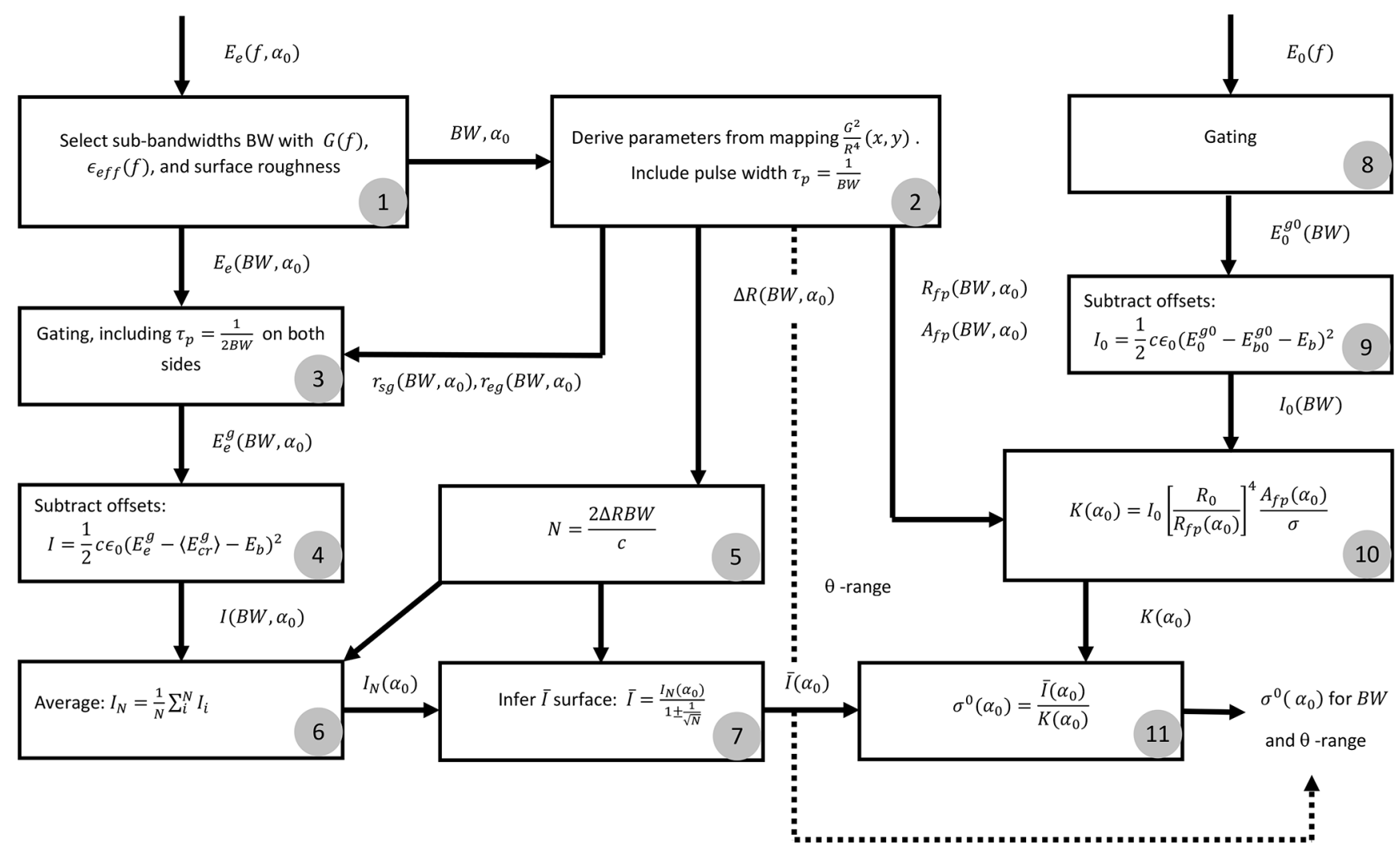

Figure 4. Flow chart of $\sigma^{0}$ derivation process. Inputs are the measured backscattered electric fields of the surface target $E_{\mathrm{e}}\left(f, \alpha_{0}\right)$ and the calibration standard $E_{0}(f)$. The process follows from 1 to 11 in sequence.

of offset subtraction from $E_{\mathrm{e}}^{\mathrm{g}}$ was done in for example Nagarajan et al. (2014). Next, the result is squared and converted into intensity $I\left(\mathrm{BW}, \alpha_{0}\right)\left(\mathrm{W} \mathrm{m}^{-2}\right)$.

5. To reduce the radiometric uncertainty due to fading we perform frequency averaging. The number of statistically independent frequency samples $N$ within BW is calculated with $\Delta R=r_{\mathrm{eg}}-r_{\mathrm{sg}}(\mathrm{m})$. Please refer to Appendix Sect. C2 for more information.

6. From the $I\left(\mathrm{BW}, \alpha_{0}\right)$ spectrum $N$ intensities are selected at equidistant intervals of $\Delta f=\mathrm{BW} / N-1(\mathrm{~Hz})$ and averaged to $I_{N}\left(\alpha_{0}\right)$.

7. With $I_{N}\left(\alpha_{0}\right)$ and $N$, the average received intensity $\bar{I}\left(\alpha_{0}\right)$ $\left(\mathrm{W} \mathrm{m}^{-2}\right)$ is calculated using Eq. (C4). The denominator $1 \pm 1 / \sqrt{N}$ implies that $\bar{I}$ is estimated with a $68 \%$ confidence interval.

8. The gated backscattered signal from the reference target $E_{0}^{\mathrm{g} 0}(\mathrm{BW})\left(\mathrm{V} \mathrm{m}^{-1}\right)$ (subscript 0 represents "reference"; superscript g0 stands for "gate" during reference measurements) is determined for the full $0.75-10.25 \mathrm{GHz}$ band under the assumption that $G \approx 1$ for all frequencies (see Appendix Sect. B4). After gating the relevant $\mathrm{BW}$ of $E_{0}^{\mathrm{g} 0}$ is selected.
9. The measured response from the mast without reference target $E_{\mathrm{b} 0}^{\mathrm{g} 0}(\mathrm{BW})\left(\mathrm{Vm}^{-1}\right)$ is subtracted from the reference target response. Subscript b0 denotes background calibration, and the superscript $\mathrm{g} 0$ indicates that the same gate was used as with the reference target response. Also $E_{\mathrm{b}}$ is subtracted here. The result is squared and converted into intensity $I_{0}(\mathrm{BW})\left(\mathrm{W} \mathrm{m}^{-2}\right)$.

10. The $I_{0}(\mathrm{BW})$ is used to calculate the factor $K\left(\mathrm{~W} \mathrm{~m}^{-2}\right)$, given the footprint area $A_{\mathrm{fp}}$ and centre distance $R_{\mathrm{fp}}$ (Eq. C2).

11. The final step is the application of Eq. (C1) with $\bar{I}\left(\alpha_{0}\right)$ and $K\left(\alpha_{0}\right)$ as inputs to obtain $\sigma^{0}$. By steps 2 and 6 the derived $\sigma^{0}$ is to be associated with the chosen BW and calculated $\theta$ range. By step 7 a $68 \%$ confidence interval applies to $\sigma^{0}$.

\section{Measurement results}

For the analyses in this paper we discuss results of four bandwidths BW, picked amidst frequency ranges typically used in microwave remote sensing: $9-10 \mathrm{GHz}$ (X-band), $4.5-5 \mathrm{GHz}$ (C-band), 2.5-3 GHz (S-band), and 1.5-1.75 GHz (L-band). The widths decrease with wavelength due to the expected 
frequency resolution of the target's scattering response (Appendix Sect. C2) and the antenna-radiation-pattern change over frequency (Appendix Sect. B4). Presented in this section is, first, a global overview of the retrieved $\sigma_{p q}^{0}$ over the period 26 August 2017-26 August 2018, followed by a $13 \mathrm{~d}$ time series of $\sigma_{p q}^{0}$ at the highest temporal resolution during the thawing period in April 2018.

Figure 5 presents an overview of the time-series data of $\sigma_{p q}^{0}$ over the whole August 2017-2018 period for all considered bandwidths in L-, S-, C-, and X-band, along with $M_{\mathrm{V}}$ and $T_{\text {soil }}$ at four depths ranging from 2.5 to $20 \mathrm{~cm}$ and precipitation. Based on observed albedo values, days at which a layer of snow was present are indicated. For visibility reasons the graphs only display measurements taken at 18:10 LT with $2 \mathrm{~d}$ intervals and one cross-polarization channel $\left(\sigma_{\mathrm{vh}}^{0}\right.$ and $\sigma_{\text {hv }}^{0}$ are within each other's confidence intervals). Data of the radar return and $\sigma_{p q}^{0}$ for November 2017 are not available, while those of late June-Early July 2018 will become available at a later stage.

We observe for all bands and polarizations that $\sigma^{0}$ is highest in summer and autumn, while it is lowest during winter. The same observations were made with satellites over the Maqu area for L-band (Wang et al., 2016) and C-band (Dente et al., 2014). This behaviour can be explained by the fact that in summer and autumn $M_{\mathrm{v}}$ and the amount of fresh biomass is highest. As a result, the high dielectric constant of moist soil in combination with the rough surface and presence of water in the vegetation results in strong backscattering. During winter, however, there is little liquid water, i.e. $M_{\mathrm{v}}$, present in the soil and no fresh biomass (dry biomass however remains present; see Fig. A1). Black arrows indicate frozen and thawed soil at $25 \mathrm{~cm}$ depth (Appendix Sect. A2). The dielectric constant of the soil therefore is lower compared to that of moist soil, and there is little to no scattering from the dried out vegetation, resulting in a lower $\sigma_{p q}^{0}$. All aforementioned effects are described in, for example, (Ulaby and Long, 2017). There were, however, also peaks of $\sigma_{p q}^{0}$ during winter, for example on 26 January, which coincided with snowfall. In (Lin et al., 2016) strong backscatter increments due to fresh snowfall were also observed for X-band. Apparently, this behaviour is similar with the longer wavelengths as the graphs show.

When comparing the four bands we observe that, in general, the backscattering is highest for X-band and lowest for L-band or S-band. This difference is mainly driven by the wavelength-dependent response to the surface roughness of the soil and vegetation during the summer and autumn period. For longer wavelengths the soil surface roughness appears smoother than for the shorter wavelengths, resulting in stronger specular reflection, thus lower backscatter. A similar argument holds for the vegetation: its constituents are small compared to the longer wavelengths; thus little volume scattering occurs.

Except for during the summer, backscatter for vv polarization was equal to or higher than that for hh polarization.
This behaviour was also observed by Oh et al. (1992), albeit for bare soil. We, however, may compare our situation to that of bare soil during winter, when there is no fresh biomass. When vegetation was present, $\sigma_{\text {hh }}^{0}$ was stronger for all bands, as is visible during June-August 2018. This was however not the case during August-September 2017, when the vegetation probably still contained water. Somewhat stronger backscatter, $0.5-1 \mathrm{~dB}$, for hh than for vv polarization was also reported for grassland in Ulaby and Dobson (1989) with $40 \leq \theta \leq 60^{\circ}$ for S- and X-band. For C-band they reported no clear difference. Yet another study, (Kim et al., 2014), measured 3-4 dB higher backscatter for hh than for vv polarization when measuring wheat at L-band $\left(\theta=40^{\circ}\right)$. Our results for L-band were similar. Cross-polarization $\sigma^{0}$ levels were, as expected, lower than those of co-polarization. During the winter period this difference was largest, especially with C-band. For L-band, on the other hand, this difference in $\sigma^{0}$ levels between co-polarization and cross polarization was quite small.

Next, four $13 \mathrm{~d}$ time series of $\sigma^{0}$ at $30 \mathrm{~min}$ intervals are presented. When selecting these periods we tried avoiding strong precipitation events as much as possible, since these complicate the interpretation. In Appendix Sect. D time series during October 2017 (Fig. D1), December 2017 (Fig. D2), and July 2018 (Fig. D3) can be found. Here we shall describe the retrieved $\sigma_{p q}^{0}$ during a $13 \mathrm{~d}$ period in April 2018 (Fig. 6) when the thawing process was ongoing.

The most prominent features in Fig. 6 are the diurnal variations of $\sigma_{p q}^{0}$ that are clearly caused by changes in $M_{\mathrm{v}}$. For $\mathrm{S}$-, C-, and X-bands we observe that $\sigma^{0}$ increases during daytime due to the increase in liquid water in the top soil due to thawing, and at night $\sigma^{0}$ drops as most of the water freezes again. For L-band this behaviour is also visible, though not as pronounced. The $M_{\mathrm{v}}$ changes at different depths are consistent with this difference: the strongest diurnal variation in liquid water was measured by the probes at 2.5 and $5 \mathrm{~cm}$ depth, while those at 10 and $20 \mathrm{~cm}$ do not change as much. On some days, for example on 4 and 5 April, or on 10 April, we observe diurnal changes in $\sigma^{0}$ (most pronounced for X-band), while the $M_{\mathrm{v}}$ measured by the $5 \mathrm{TM}$ sensors at 2.5 and $5 \mathrm{~cm}$ depth showed little variation. This may suggest that the freezing and thawing during those days occurred only in the very top soil layer, just below the air-soil interface where it was outside the influence zone of the 5TM sensors. The time lag between the drop of $\sigma^{0}$ (first) and the drop of 5TM $M_{\mathrm{v}}$ (second) is caused by the same phenomena as the freezing starts at the top soil layer and progresses downward. The time lag during thawing was smaller. In general the magnitude of the $\sigma^{0}$ change was largest for X-band and smallest for L-band, though exceptions exist. See for example 3 April, where for L-band $\sigma_{\text {hh }}^{0}$ drops almost $10 \mathrm{~dB}$, which is more than for other bands. At the same time $M_{\mathrm{v}}$ at $20 \mathrm{~cm}$ depth also shows strong variation, while $M_{\mathrm{v}}$ at $10 \mathrm{~cm}$ changes less. 


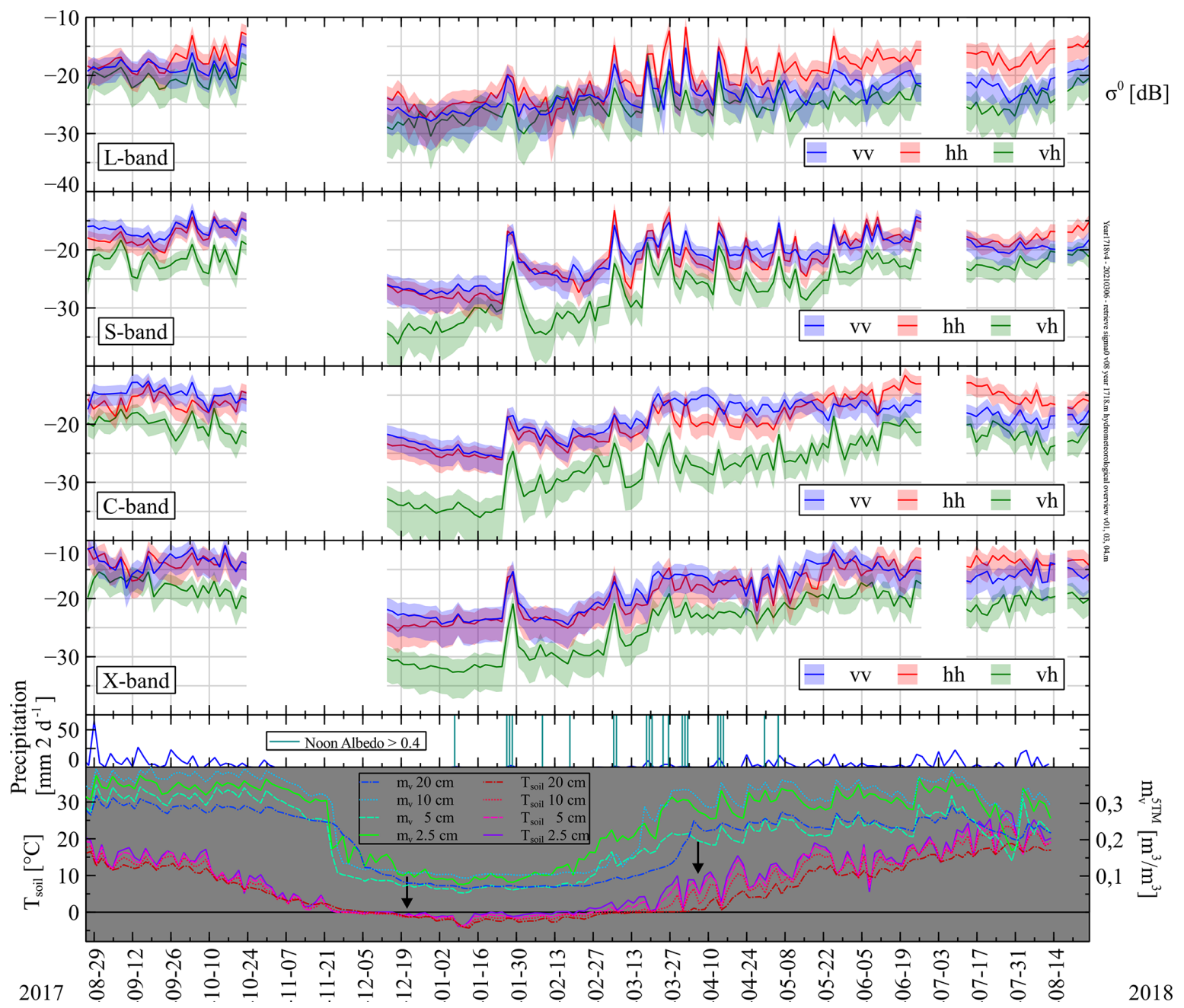

Figure 5. Time-series measurements of $\sigma_{p q}^{0}\left(\mathrm{~m}^{2} \mathrm{~m}^{-2}\right)$ for L-, S-, C-, and X-band, $M_{\mathrm{V}}$, and $T_{\text {soil }}$ from August 2017 to 2018 . Shown are measurements taken at 18:10 LT with $2 \mathrm{~d}$ intervals. Shaded regions indicate $66 \%$ confidence intervals for $\sigma_{p q}^{0}$. The antenna boresight angle was fixed at $\alpha_{0}=55^{\circ}$. The incidence angle ranges were band and polarization dependent. The widest ranges were $0^{\circ} \leq \theta \leq 60^{\circ}$ for $\mathrm{L}$ band, $20^{\circ} \leq \theta \leq 60^{\circ}$ for S-band, $36^{\circ} \leq \theta \leq 60^{\circ}$ for C-band, and $47^{\circ} \leq \theta \leq 59^{\circ}$ for X-band. Bottom graphs show measured precipitation per $2 \mathrm{~d}$ (snowfall identified by noon albedo), volumetric soil moisture $m_{\mathrm{v}}^{5 \mathrm{TM}}\left(\mathrm{m}^{3} \mathrm{~m}^{-3}\right)$, and soil temperature $T_{\text {soil }}$ at indicated depths. Arrows indicate frozen/thawed soil at $25 \mathrm{~cm}$. Spatial average volumetric soil moisture $M_{\mathrm{V}}$ is estimated as $M_{\mathrm{V}}=m_{\mathrm{V}}^{5 \mathrm{TM}} \pm 0.04 \mathrm{~m}^{3} \mathrm{~m}^{-3}$.

\section{Discussion}

\subsection{Reference measurements for asphalt}

In order to check our scatterometer setup and $\sigma^{0}$ retrieval procedure an experiment was performed in which the backscatter of asphalt was measured and subsequently compared to results found in other studies. This exercise is described in Appendix Sect. F. We found that our results for $\mathrm{X}$-band with co-polarization and S-band for vv and vh polarization match with those reported in Ulaby and Dobson (1989) and Baldi (2014) respectively. For L-band a proper comparison was not possible due to the width of our antenna patterns. We could not find other studies reporting backscatter for C-band to compare our results to.

\subsection{Measurement uncertainty}

In the derivation of $\sigma^{0}$ we distinguish four sources of uncertainty: (i) fading (Sect. 3.2.3), (ii) the temperature-induced radar return uncertainty $\Delta E_{T}\left(\mathrm{Vm}^{-1}\right)$, (iii) reference target measurement uncertainty $\Delta K$ (in $\mathrm{dB}$, as it is a relative value), and (iv) the low-directivity-induced uncertainty.

First we describe (ii) and (iii), which are systematic sources of uncertainty. In this context we also consider the system's offsets levels formed by the antenna-to-antenna coupling remnant $E_{\mathrm{cr}}^{\mathrm{g}}\left(\mathrm{Vm}^{-1}\right)$ and the minimum signal strength measurable by the VNA, or background $E_{\mathrm{b}}\left(\mathrm{V} \mathrm{m}^{-1}\right)$. The former is derived from measurements with the antennas aimed skywards. From $E_{\mathrm{b}}$ the minimum measurable RCS (given a certain distance $R$ to target) $\sigma_{\min }$ can be calculated 


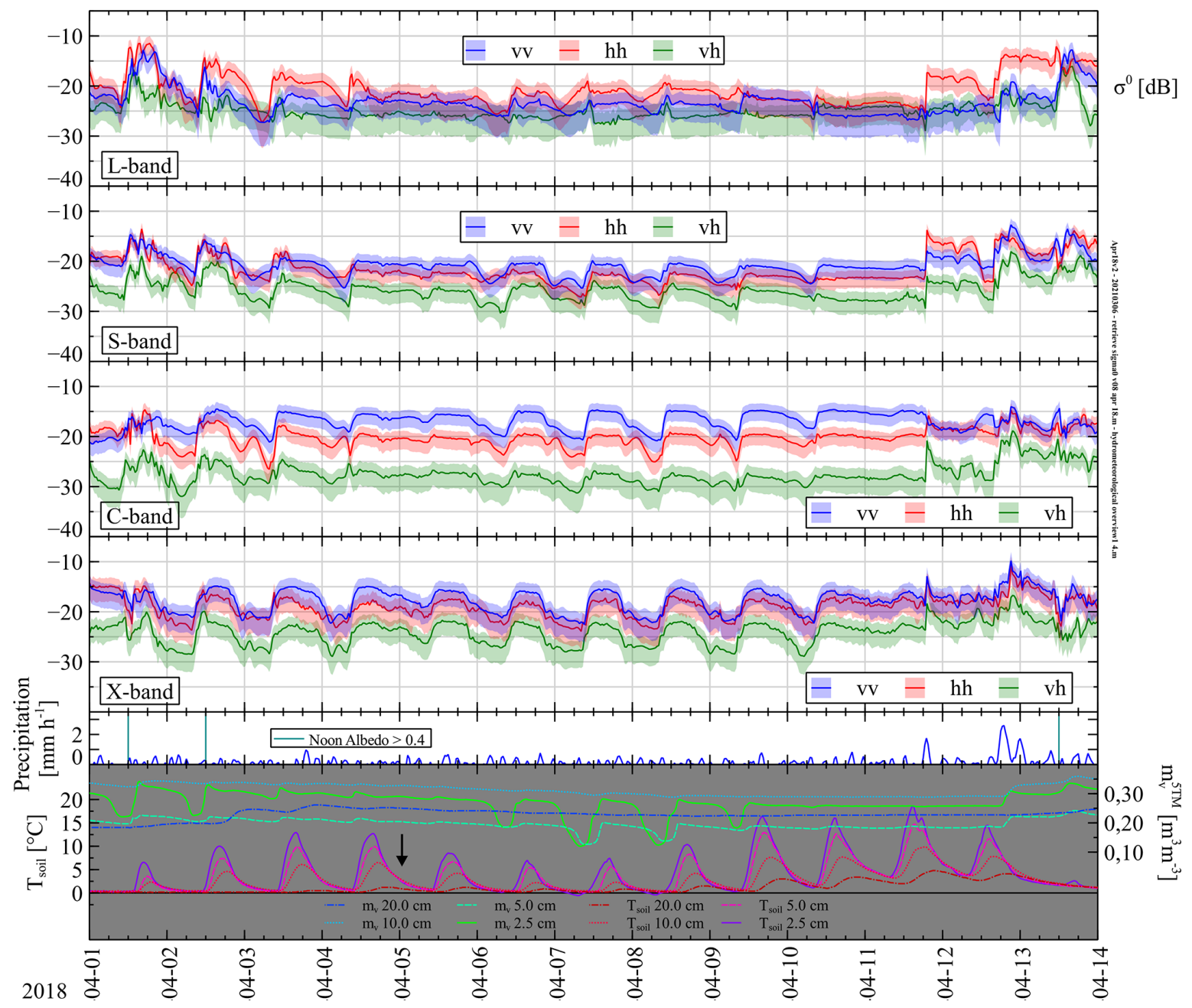

Figure 6. Time-series measurements of $\sigma_{p q}^{0}\left(\mathrm{~m}^{2} \mathrm{~m}^{-2}\right)$ for L-, S-, C-, and X-band, precipitation, $M_{\mathrm{V}}$, and $T_{\text {soil }}$ during $13 \mathrm{~d}$ in April 2018. Shaded regions indicate $66 \%$ confidence intervals for $\sigma_{p q}^{0}$. The antenna boresight angle was fixed at $\alpha_{0}=55^{\circ}$. The incidence angle ranges were band and polarization dependent. The widest ranges were $0^{\circ} \leq \theta \leq 60^{\circ}$ for L-band, $20^{\circ} \leq \theta \leq 60^{\circ}$ for S-band, $36^{\circ} \leq \theta \leq 60^{\circ}$ for $\mathrm{C}$ band, and $47^{\circ} \leq \theta \leq 59^{\circ}$ for X-band. Bottom graphs show measured precipitation $\left(\mathrm{mm} \mathrm{h}^{-1}\right.$ ) (snowfall identified by noon albedo), volumetric soil moisture $m_{\mathrm{V}}^{5 \mathrm{TM}}\left(\mathrm{m}^{3} \mathrm{~m}^{-3}\right)$, and soil temperature $T_{\text {soil }}$ at indicated depths. Arrow indicates thawing of soil at $25 \mathrm{~cm}$. Spatial average volumetric soil moisture content $M_{\mathrm{v}}$ is estimated as $M_{\mathrm{v}}=m_{\mathrm{v}}^{5 \mathrm{TM}} \pm 0.04 \mathrm{~m}^{3} \mathrm{~m}^{-3}$.

via Eq. (3), where instead of the product $\sigma^{0} A_{\mathrm{fp}}$ a RCS value is to be calculated using the power levels associated with $E_{\mathrm{b}}$. Appendix Sect. E contains detailed information on all considered systematic sources of uncertainty and offsets, starting with an overview (Appendix Sect. E1), followed by sections on $\Delta E_{T}$ (Appendix Sect. E2), $\Delta K$ (Appendix Sect. E3), and $E_{\mathrm{cr}}^{\mathrm{g}}(f)$ (Appendix Sect. E4).

Starting with Eq. (C1) it can be shown (see Appendix Sect. E5) that the three estimated types of uncertainty, namely fading, temperature-induced radar return uncertainty $\left(\Delta E_{T}\right)$, and reference target measurement uncertainty $(\Delta K)$, can be combined in a model for total $\sigma^{0}$ uncertainty:

$\sigma^{0}=\frac{I_{N} \pm \Delta I_{N}}{\left(K \pm \frac{2}{3} \Delta K\right)(1 \pm 1 / \sqrt{N})}=\frac{I_{N}}{K} \pm \Delta \sigma^{0}$.
$\Delta I_{N}\left(\mathrm{~W} \mathrm{~m}^{-2}\right)$ is a statistical error that follows from $\Delta E_{T}$, $\Delta K$ is converted from a maximum possible error into a statistical error with a $(2 / 3)$ probability confidence interval, and the term $1 / \sqrt{N}$ represents a statistical error caused by fading. In the right term the three uncertainty contributions are merged into one statistical uncertainty $\Delta \sigma^{0}\left(\mathrm{~m}^{2} \mathrm{~m}^{-2}\right)$, which is a $66 \%$ confidence interval for $\sigma_{0}$. In this paper these $66 \%$ confidence intervals are presented in all figures showing our retrieved $\sigma^{0}$. To give an indication of the magnitude of $\Delta \sigma^{0}$, some typical values over band, polarization, and season are summarized in Table 2. Presented values were retrieved from the calculated time-series results of Sect. 4.

The low-directivity-induced uncertainty (iv) is not quantifiable in the sense that with the time-series experiments backscatter was not repeatedly measured at different $\alpha_{0}$ an- 
Table 2. Example uncertainty values $\Delta \sigma^{0}(\mathrm{~dB})$ per bandwidth, polarization, and overall $\sigma^{0}$ level.

\begin{tabular}{ccccc}
\hline \multicolumn{2}{c}{ L-band } & S-band & C-band & X-band \\
\hline \multicolumn{4}{c}{ High $\sigma^{0}$ levels (typical in summer) } \\
\hline vv $\quad+1.6$ to -2.5 & +1.3 to -1.9 & +1.4 to -2.1 & +1.7 to -3.0 \\
vh $\quad+1.7$ to -3.0 & +1.3 to -1.9 & +1.4 to -2.2 & +1.6 to -2.7 \\
hv $\quad+1.8$ to -3.2 & +1.3 to -1.9 & +1.4 to -2.0 & +1.6 to -2.7 \\
hh $\quad+1.6$ to -2.5 & +1.2 to -1.7 & +1.3 to -2.0 & +1.7 to -2.9 \\
\hline Low $\sigma^{0}$ levels (typical in winter) & & \\
\hline vv $\quad+2.3$ to -5.2 & +1.9 to -3.7 & +1.7 to -2.9 & +2.1 to -4.2 \\
vh $\quad+2.3$ to -5.2 & +2.4 to -5.9 & +2.6 to -8.3 & +2.3 to -5.2 \\
hv $\quad+2.4$ to -6.0 & +2.5 to -6.6 & +2.5 to -6.4 & +2.0 to -4.9 \\
hh $\quad+2.3$ to -5.3 & +1.7 to -2.8 & +1.7 to -2.7 & +1.9 to -3.8 \\
\hline
\end{tabular}

gles. With such measurements, sets of $P_{q}^{\mathrm{RX}}\left(\alpha_{0}\right)$ would be obtained that can be deconvolved into $\sigma^{0}(\theta)$, since $G(\alpha, \beta)$ is known (see Eq. 2). This deconvolution approach was performed by, for example, Axline (1974) and Ulaby et al. (1983). It is possible, however, to give an estimate of the lowdirectivity-induced uncertainty, inherent to our $\sigma^{0}$ retrieval method, with a simple numerical experiment in which the scatterometer radar return is simulated (Eq. 2) using a predefined function for $\sigma^{0}(\theta)$. We may use for example the empirical model of $\sigma_{p q}^{0}(\theta)$ for grassland developed in Ulaby and Dobson (1989) with measurement data from several other studies. Applying the method of Sect. 3.2.3 on the simulated radar return, we obtain for $4.75 \mathrm{GHz}$ at vv polarization $\sigma_{\mathrm{vv}}^{0}=-14.4 \mathrm{~dB}$ for $34^{\circ} \leq \theta \leq 60^{\circ}$, while the actual value over this interval varies from $-13.0 \leq \sigma_{\mathrm{vv}}^{0} \leq-14.9 \mathrm{~dB}$. Although this discrepancy depends on the (unknown) form of $\sigma^{0}(\theta)$, in general this error will be larger for low frequencies and smaller for high frequencies because of the respective antenna beamwidths, which has to be kept in mind when using the $\sigma^{0}$ values of this dataset. Despite this uncertainty, the $\sigma^{0}$ retrieved in this dataset nevertheless does show all relevant temporal dynamics that are furthermore wavelength and polarization dependent.

Alternatively, the low-directivity-induced uncertainty can be avoided by using the radar return of the dataset $P_{p}^{\mathrm{RX}}$ together with a microwave scattering model instead of the retrieved $\sigma^{0}$. The angle-dependent $\sigma_{p q}^{0}(\theta)$ then may be obtained by the microwave scattering model and simply applied in Eq. (2) to simulate the radar return, which subsequently can be compared to the measured $P_{p}^{\mathrm{RX}}$ values.

\subsection{Angular variation of $\sigma_{p q}^{0}$ in Maqu}

Next, we present the measurement results and analysis of the angle-dependent backscatter of the Maqu site surface for two purposes. First, we present it to quantify the behaviour of $\sigma^{0}$ with respect to the elevation angle $(\theta), \mathrm{BW}$, and polarization channels for the Maqu site ground surface with a living vegetation canopy, and, second, we present it to assess the spatial homogeneity of $\sigma^{0}(\theta)$ over the Maqu site surface by also measuring backscatter at different azimuth angles $(\phi)$. As explained in Appendix Sect. C2, the single footprint area for the $\sigma^{0}$ time-series measurements should be representative for the whole Maqu site surface. Due to practical limitations of possible $\phi$ angles and because of the wide antenna beamwidths, the footprints of used $\alpha_{0}$ and $\phi$ combinations in this experiment overlap partially, as is shown in Fig. 2. However, since we employ frequency averaging to reduce the fading uncertainty for every footprint, we argue that the $\sigma^{0}$ values retrieved per (overlapping) footprint may nevertheless be compared to each other for this section's analysis.

As a means to quantitatively evaluate the $\sigma^{0}$ behaviour with respect to the $\theta$ and $\phi$ angle, the data are grouped in sets of $\sigma^{0}$ over $\alpha_{0}$ for every angle $\phi$, BW, and polarization. In Appendix Sect. G, Fig. G1 examples of such sets are shown. Next, an iterative least-squares non-linear fitting algorithm is applied to fit each set to the model:

$\sigma^{0}=A \cos (\theta)^{B}$,

where $A$ is a constant $\left(\mathrm{m}^{2} \mathrm{~m}^{-2}\right)$ and $B$ is either 1 for an isotropic scatterer or 2 for a surface in accordance with Lambert's law (Clapp, 1946). For each $\alpha_{0}$ we find the coordinate for which $G^{2} / R^{4}$ is maximum and use that position's angle of incidence $\theta$ together with the centre $\sigma^{0}$ value of the $66 \%$ confidence interval for the fitting process. As a next step, we reduced the number of fitting possibilities by selecting for each polarization-BW combination the most likely value for $B$ (1 or 2). This was done by tallying over the $\phi$ angles which of the two fitted curves $\sigma^{0}=A \cos (\theta)^{B}$ passed through the confidence intervals best and had the highest coefficients of determination $\left(R^{2}\right)$. The outcome was $B=1$ for all polarization channels of X-band and $B=2$ for all of S- and L-band. For C-band it was harder to judge in favour of either. We chose $B=1$ for vh polarization and $B=2$ for $\mathrm{vv}, \mathrm{hh}$, and hv. An overview for found parameters $A$ and $B$ is presented in Fig. 7. The stronger decrease over angle found with Land $\mathrm{S}$-band $(B=2)$ is as expected since for longer wavelengths there is less volume scattering from the vegetation canopy and the soil reflections become more dominant. For these longer wavelengths the soil surface roughness appears smoother, causing specular reflection to be stronger and nonspecular reflections (including in the backward direction) to decrease more rapidly with $\theta$. This effect is well known; see for example de Roo and Ulaby (1994). By the same logic, for X-band $\sigma^{0}$ will decrease more slowly over $\theta(B=1)$ as scattering from the vegetation canopy becomes dominant over that from the soil surface. Strong vegetation scattering is known to be more constant over $\theta$ (see for example Stiles et al., 2000), and thus the model for an isotropic scattering surface, i.e. $B=1$, is more suitable. With $\mathrm{C}$-band both $B=1$ and $B=2$ fitted best for about half of the $\phi$ angles, which indicates that at this intermediate wavelength we see 

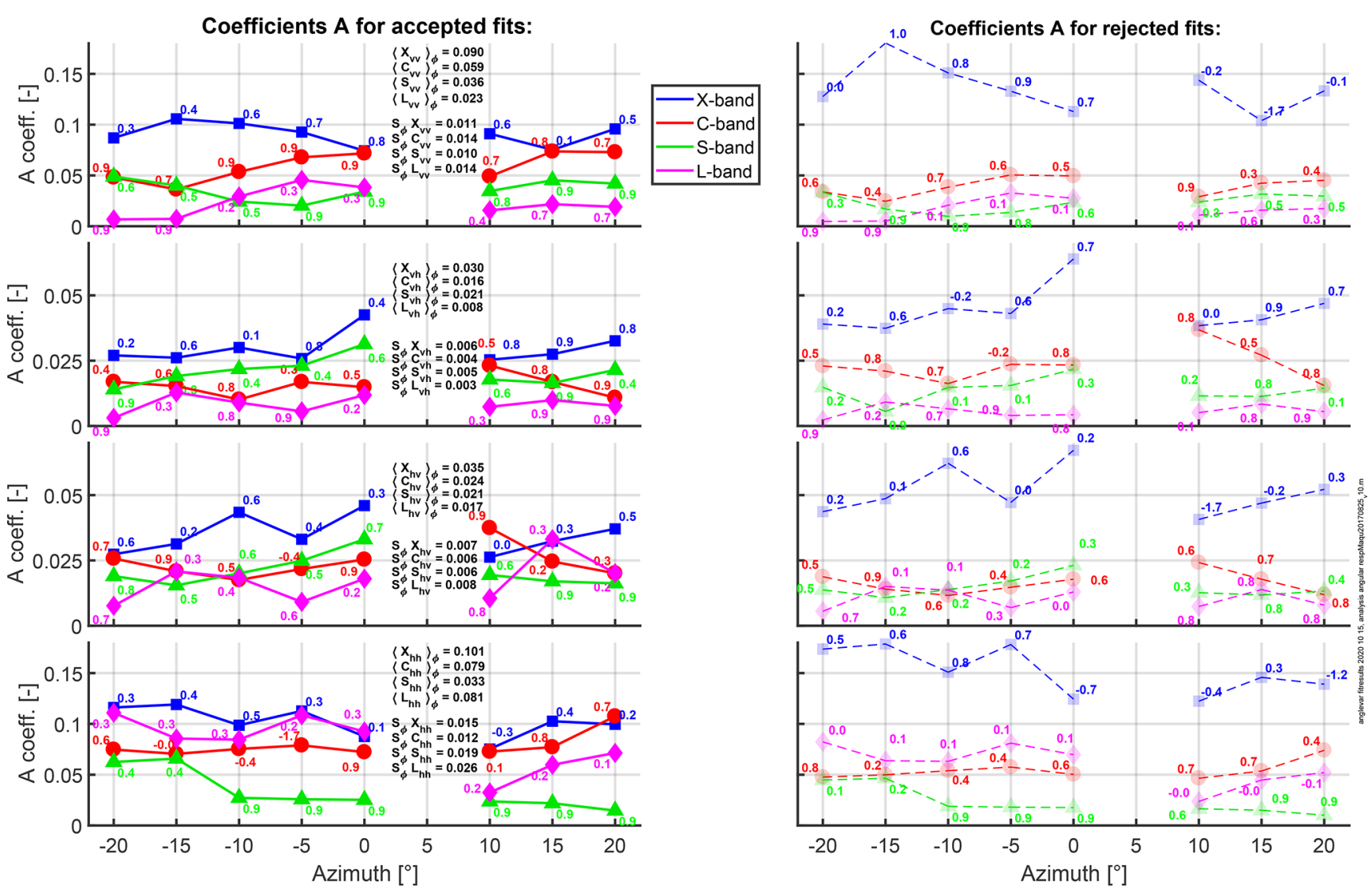

Figure 7. Results of fitting the derived values $\sigma_{p q}^{0}$ over $\alpha_{0}$ to model $\sigma_{0}(\theta)=A \cos (\theta)^{B}$ for different azimuth angles $\phi$, bandwidths BW, and polarization channels. The left column shows found coefficients $A$ over $\phi$ for best fits with the favourable $B$ value for each BW and polarization, and the right column shows the $A$ coefficients with the less favourable $B$ values. Numbers at data points indicate coefficient of determination $\left(R^{2}\right)$ of individual fits. Values in the centre are average $\left\langle B_{p q}\right\rangle_{\phi}$ and standard deviation $S_{\phi} B_{p q}$ over $\phi$, with $B=L, S, C$, or $X$ as bandwidth.

both aforementioned features. With the co-polarization channels we see that the average $A$ values over $\phi$ decrease with increasing wavelength as expected considering the description above. An exception, however, is the L-band response with hh polarization, which is comparable to that of C-band. As with the asphalt measurements (Appendix Sect. 5.1), we believe these high $\sigma^{0}$ retrievals are due to the low angular resolution of our scatterometer for L-band. As a result, the backscatter for close to nadir angles (which are highest in general) is present in all angular positions $\alpha_{0}$. This is visible in the inset figure of Fig. G1. We also note that the variation over $\phi$ (by comparing $S_{\phi} B_{p p}$ to $\left\langle B_{p q}\right\rangle_{\phi}$ ) is smallest for $\mathrm{X}$ band and largest for L-band. The cross response is lower than that for the co-polarization as expected. For both vh and hv the X-band backscatter is also largest here, while the crosspolarization backscatter for L-band is lowest. However, Sband appears to have stronger backscatter than C-band. We do not have a clear explanation for this. As with the copolarization channels the variation over $\phi$ is strongest for the longer wavelengths.
Finally some remarks on the variation of $A$ over $\phi$ and, virtually, across the surface area. Except for X-band with hh polarizations there did not appear to be a systematic trend of $A$ over $\phi$. Also, there was not one particular $\phi$ angle for which the values for $A$ over BW and polarization stood out from the rest. These observations indicate that the surface area covered by our scatterometer appeared to have uniform (scattering) properties. The somewhat higher $A$ values with the negative $\phi$ values with X-band at hh polarization are probably caused by a difference in vegetation density between the left and right side of the Maqu site. Fortunately, for $\phi=0^{\circ}$ the $A$ value had a medium value compared to the other $\phi$ angles, so that we may still interpret the surface area associated with the scatterometer's (fixed) footprint during the time-series measurements as being representative for its surroundings.

\section{Code and data availability}

In the DANS repository, under the link https://doi.org/10.17026/dans-zfb-qegy, the collected scatterometer data are publicly available (Hofste et al., 
2021). Stored are both the radar return amplitude and phase for all four linear polarization combinations and processed $\sigma_{p q}^{0}$ for the L-, S-, C-, and X-band bandwidths discussed in this paper. The dataset includes time-series measurements from 26 August 2017-26 August 2018, data of angular-variation experiments, and radar returns of the reference targets. Accompanying data include time-series measurements of soil moisture and temperature profile at depths of $[2.5,5.0,7.5,10, \ldots 90,100 \mathrm{~cm}]$, as well as time-series measurements of air temperature, precipitation and up- and downward short- and long-wave irradiation. Note that the volume of the dataset is too large $(20 \mathrm{~GB})$ to disseminate via DANS' web interface. Users are to contact the DANS repository, after which DANS will establish an alternate file transfer. Also, in the DANS repository under https://doi.org/10.17026/dans-xyf-fmkk (Hofste, 2021), MATLAB scripts are available for processing measured radar return data and for retrieving $\sigma_{p q}^{0}$ for other bands within the measured $1-10 \mathrm{GHz}$ frequency range.

\section{Conclusions}

A ground-based scatterometer system was installed on an alpine meadow over the Tibetan Plateau and collected a 1-year dataset of microwave backscatter over a broad 1$10 \mathrm{GHz}$ band for all four linear polarization combinations.

Measurements of the incidence angle dependence of $\sigma_{p q}^{0}$ for asphalt agreed with previous findings, thereby showing our $\sigma^{0}$ retrieval method to be accurate. Presented analysis on the angle-variation data of $\sigma^{0}$ in Maqu showed wavelengthand polarization-dependent scattering behaviour due to vegetation that is in accordance with theory and other studies. Furthermore, these measurements indicated the Maqu ground surface to have spatially homogeneous electromagnetic properties and the area associated with the (fixed) footprint for the time-series measurements to be representative of its surroundings.
The uncertainty of our retrieved $\sigma^{0}$ consists of quantifiable parts estimated from fading and systematic measurement uncertainties and an unknown part due to the low directivity of used antennas. The quantifiable uncertainty in $\sigma^{0}$ was estimated with an error model providing $66 \%$ confidence intervals that are different over frequency bands, polarizations, and the overall level of the radar return. Typical $\Delta \sigma^{0}$ values during summer range from $\pm 1.5 \mathrm{~dB}$ for S-band with hh polarization to $\pm 2.5 \mathrm{~dB}$ for L-band with hv polarization. Despite aforementioned uncertainties in $\sigma^{0}$ we believe that the strength of our approach lies in the capability of measuring $\sigma^{0}$ dynamics over a broad frequency range, $1-10 \mathrm{GHz}$, with high temporal resolution over a full-year period.

Our preliminary analysis on the retrieved $\sigma_{p q}^{0}$ for L-, S-, $\mathrm{C}$-, and X-band demonstrates that the scatterometer dataset collected at fixed time intervals over a full year at the Maqu site contains valuable information on exchange of water and energy at the land-atmosphere interface - information which is difficult to quantify with in situ measurement techniques alone. Hence further investigation of this scatterometer dataset provides an opportunity to gain new insights in hydrometeorological processes such as freezing and thawing, or wavelength-dependent scattering effects in the vegetation canopy during spring and summer periods. 


\section{Appendix A: Results supporting measurements}

\section{A1 Photographs of the site phenology}

In this section we present a set of photographs (see Fig. A1) of the Maqu site taken at different seasons since the installation of the ELBARA-III in January 2016. These may give the reader a global indication of how the site phenology changes throughout the seasons.

\section{A2 Hydrometeorological sensors and measurement results}

Table A1 lists all hydrometeorological instruments used for this study along with their reported measurement uncertainties. Air temperature was measured with a platinum resistance thermometer, type HPM 45C, installed $1.5 \mathrm{~m}$ above the ground, and precipitation (both rain and snow) was measured with a weight-based rain gauge, type T-200B.

We formulate in brief our main observations over the measured hydrometeorological quantities at the Maqu site over the period 26 August 2017-26 August 2018. Figure A2 provides an overview with a $2 \mathrm{~d}$ temporal resolution. All data are available in the dataset with a temporal resolution of $30 \mathrm{~min}$.

The lowest air temperatures $T_{\text {air }}$ were measured in January 2018 , during which daily minimum values dropped below $-20^{\circ} \mathrm{C}$, while daily maximum temperatures did not rise above $0^{\circ} \mathrm{C}$. In July-August $2018 T_{\text {air }}$ was highest, with maxima above $20^{\circ} \mathrm{C}$.

Soil temperature $T_{\text {soil }}$ and soil volumetric liquid water content $m_{\mathrm{v}}$ varied over depth. Depending on the amount of liquid water in the soil, the penetration depth of frozen soil at Lband can vary from $10-30 \mathrm{~cm}$ at the Maqu site (Zheng et al., 2017a). We consider $T_{\text {soil }}$ and $m_{\mathrm{v}}$ values at $25 \mathrm{~cm}$ depth, which is closest to the maximum aforementioned penetration depth. From the measurements we conclude that at $25 \mathrm{~cm}$ depth the soil can be considered frozen between 21 December 2017-5 April 2018 (arrows in figure). For other depths the freezing and thawing process is substantially different from the shown curves. During the 2017-2018 winter $T_{\text {soil }}$ dropped below $0{ }^{\circ} \mathrm{C}$ up to a depth of $70 \mathrm{~cm}$ (not shown in Fig. A2).

Total precipitation over the considered 1-year period was $688 \mathrm{~mm}$. The majority of this amount fell in the months of September and October 2017 and in August 2018, while from November 2017 to the middle of March 2018 there was only $7 \mathrm{~mm}$ precipitation. Presence of snow on soil was inferred from the observed noon albedo to be 0.4 or higher.

\section{A3 Derivation of spatial soil-moisture-variation estimate}

This section describes how the spatial average soil moisture content over the Maqu site $M_{\mathrm{v}}\left(\mathrm{m}^{3} \mathrm{~m}^{-3}\right)$ is linked to $m_{\mathrm{v}}$ as measured by the $5 \mathrm{TM}$ sensors at 2.5 and $5 \mathrm{~cm}$ depth.
At every depth, $m_{\mathrm{v}}$ varies over the horizontal spatial extent at all scales (Famiglietti et al., 2008). Local $m_{\mathrm{v}}$ variability is caused by variations in soil structure and texture, including organic matter. At the Maqu site, the 5TM sensor array forms only one spatial measurement point for soil moisture. We denote its measurements as $m_{\mathrm{v}}^{5 \mathrm{TM}}\left(\mathrm{m}^{3} \mathrm{~m}^{-3}\right)$. In an attempt to quantify how $m_{\mathrm{v}}^{5 \mathrm{TM}}$ at the top soil layer (depths 2.5 and $5 \mathrm{~cm}$ ) relates to the soil moisture over the rest of the Maqu site, we sampled $m_{\mathrm{v}}$ at 17 positions along the no-step zone (Fig. 2) on 29 June 2018 with a handheld impedance probe, type ThetaProbe ML2x, whereby three measurements were taken per position. Figure A3 shows the measured $m_{\mathrm{v}}$ in the top layer. Taking aside the outlying values at positions 1 and 15 , we observe that the variation along the periphery is slightly larger than the variability amongst the three measurements taken at a specific position. The average standard deviation over the 15 positions is $0.03 \mathrm{~m}^{3} \mathrm{~m}^{-3}$, while the average standard deviation over the three measurements is $0.02 \mathrm{~m}^{3} \mathrm{~m}^{-3}$. Given this small difference we concluded there is no clear spatial trend of top soil $m_{\mathrm{v}}$ at the Maqu site. Therefore, we considered all $15 \times 3=45$ readings as independent measurements on spatial $m_{\mathrm{v}}$ variation, which we used to determine the quantity $S_{\text {tot }}\left(\mathrm{m}^{3} \mathrm{~m}^{-3}\right)$, called the total standard deviation of spatially measured $m_{\mathrm{v}}$. $S_{\text {tot }}$ is an estimate for the spatial $m_{\mathrm{v}}$ variability over the Maqu site. Subsequently, we use $S_{\text {tot }}$ to relate the measured $m_{\mathrm{v}}^{5 \mathrm{TM}}$ to the spatial average top soil moisture content over the Maqu site $M_{\mathrm{v}}\left(\mathrm{m}^{3} \mathrm{~m}^{-3}\right)$ according to

$M_{\mathrm{v}}=m_{\mathrm{v}}^{5 \mathrm{TM}} \pm S_{\mathrm{tot}}$.

Using the assumption of temporal stability of spatial heterogeneity (Vachaud et al., 1985), we consider the found $S_{\text {tot }}$ to hold throughout the year. $S_{\text {tot }}$ is calculated by

$S_{\mathrm{t}}=\sqrt{S_{\mathrm{s}}^{2}+S_{5 \mathrm{TM}}^{2}+S_{\mathrm{p}}^{2}}$

according to standard error propagation theory (see for example Hughes and Hase, 2010). The term $S_{\mathrm{s}}\left(\mathrm{m}^{3} \mathrm{~m}^{-3}\right)$ represents the spatial $m_{\mathrm{v}}$ variability as measured along the periphery. It is calculated as the standard deviation over $45-1$ samples and is $0.031 \mathrm{~m}^{3} \mathrm{~m}^{-3}$. The standard deviation $S_{5 \mathrm{TM}}$ has value of $0.02\left(\mathrm{~m}^{3} \mathrm{~m}^{-3}\right)$ and is the root-mean-square measurement error of the 5TM sensors. It was derived in Zheng et al. (2017b) after calibrating 5TM sensor retrievals to top soil gravimetric soil samples taken at the Maqu site. The term $S_{\mathrm{p}}$ is the propagated error of the $0.05 \mathrm{~m}^{3} \mathrm{~m}^{-3}$ theta probe measurement accuracy (Table A1) when $S_{\mathrm{s}}$ is calculated. $S_{\mathrm{p}}=0.05 / \sqrt{45-1}=0.0075 \mathrm{~m}^{3} \mathrm{~m}^{-3}$. Finally, $S_{\mathrm{tot}}$ then is $0.04 \mathrm{~m}^{3} \mathrm{~m}^{-3}$. 

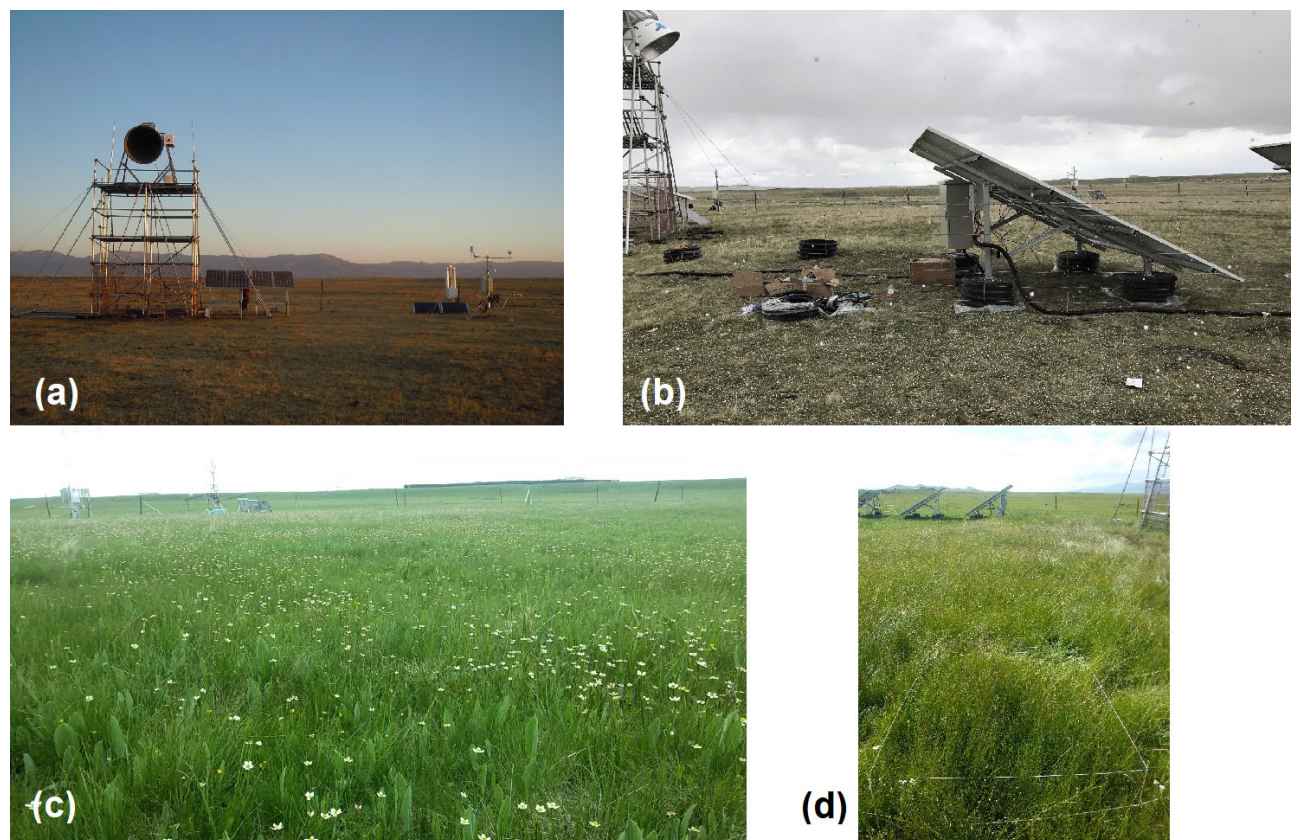

(d)
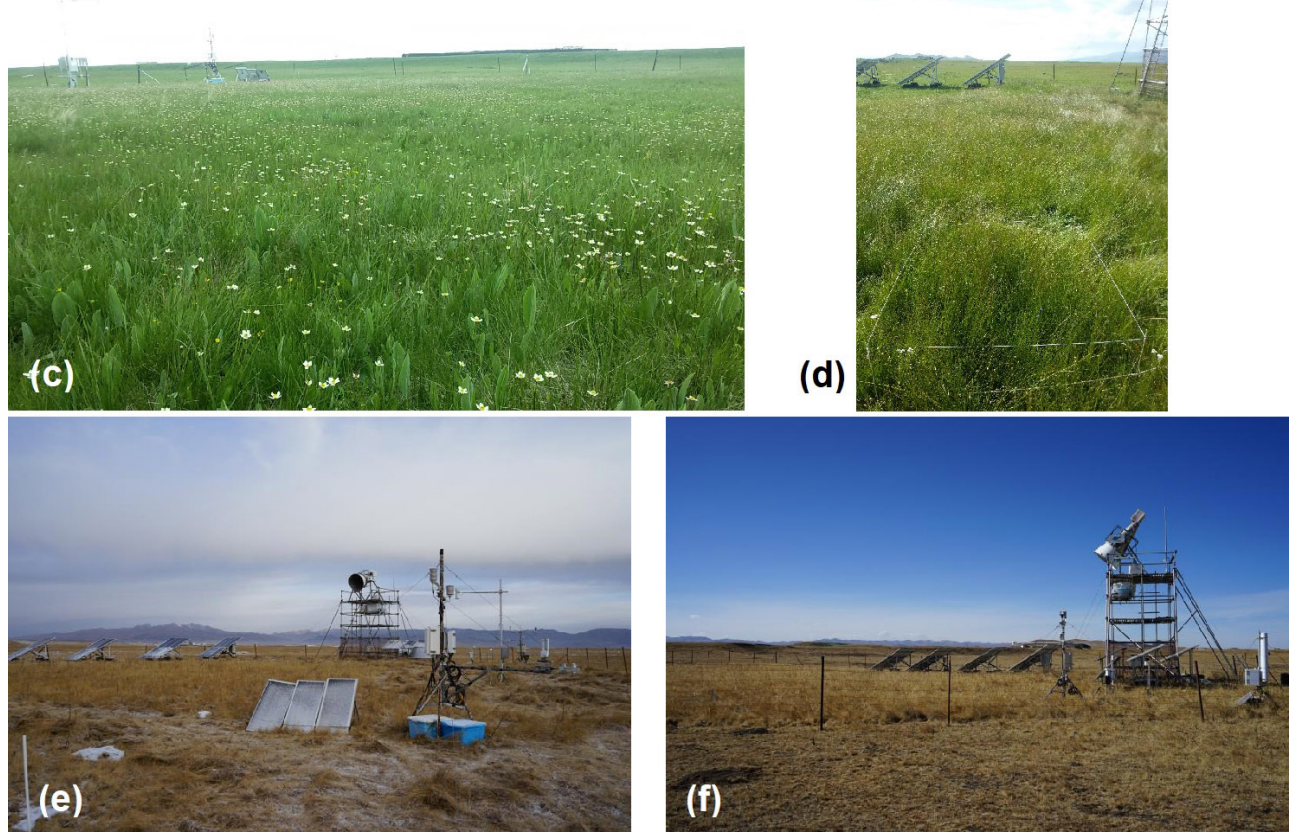

Figure A1. Maqu site changing phenology. (a) Winter, January 2016. (b) Spring, 16 May 2017. (c) Spring, 26 June 2018. (d) Summer, 17 August 2018. (e) Winter, 6 January 2018. (f) Winter, 6 January 2018.

Table A1. Overview of relevant hydrometeorological sensors at the Maqu site.

\begin{tabular}{lll}
\hline Quantity & Type, manufacturer & Unit, uncertainty \\
\hline Volumetric soil moisture $m_{\mathrm{V}}$ & 5TM, Meter Group & $\pm 0.02 \mathrm{~m}^{3} \mathrm{~m}^{-3}$ (Zheng et al., 2017b) \\
Volumetric soil moisture $m_{\mathrm{V}}$ & ThetaProbe ML2x, Delta-T Devices & $\pm 0.05 \mathrm{~m}^{3} \mathrm{~m}^{-3}$ \\
Soil temperature & 5TM, Meter Group & $\pm 1^{\circ} \mathrm{C}$ \\
Air temperature & HPM 45C, Campbell Scientific & $\pm 1^{\circ} \mathrm{C}$ \\
Precipitation (rain and snow) & T-200B, Geonor & $\pm 0.6 \mathrm{~mm}$ \\
Short- and long-wave up- and downward irradiance & NR01, Hukseflux & $\pm 5 \% \mathrm{~W} \mathrm{~m}^{-2}$ \\
\hline
\end{tabular}




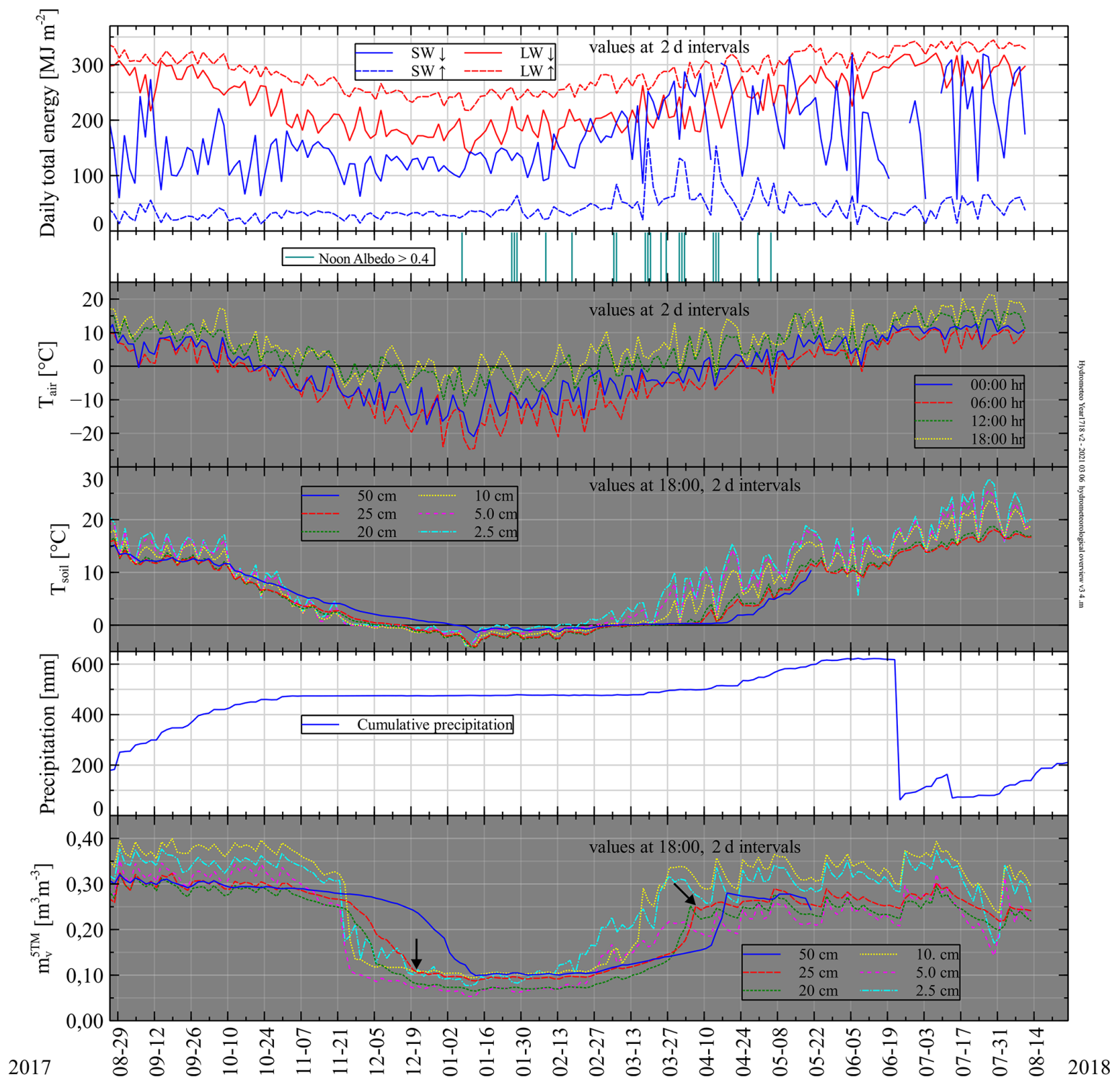

Figure A2. Overview of hydrometeorological quantities measured at the Maqu site over the period 26 August $2017-26$ August 2018. From top to bottom: daily total sum of down- and upward hemispherical energy ( $\mathrm{MJ} \mathrm{m}^{-2}$ ) for short (285-3000 nm) and long (4500$40000 \mathrm{~nm}$ ) wavelengths at $2 \mathrm{~d}$ intervals, days with snowfall (identified from noon albedo), air temperatures $\left({ }^{\circ} \mathrm{C}\right.$ ) at four times during the day at $2 \mathrm{~d}$ intervals, soil temperatures $T_{\text {soil }}\left({ }^{\circ} \mathrm{C}\right)$ for different depths at $2 \mathrm{~d}$ intervals, cumulative precipitation mm, and volumetric soil moisture $m_{\mathrm{v}}^{5 \mathrm{TM}} \mathrm{m}^{3} \mathrm{~m}^{-3}$ for different depths at $2 \mathrm{~d}$ intervals. Arrows indicate freeze/thaw of soil at $25 \mathrm{~cm}$. Spatial average volumetric soil moisture $M_{\mathrm{V}}$ is estimated as $M_{\mathrm{V}}=m_{\mathrm{v}}^{5 \mathrm{TM}} \pm 0.04 \mathrm{~m}^{3} \mathrm{~m}^{-3}$. 


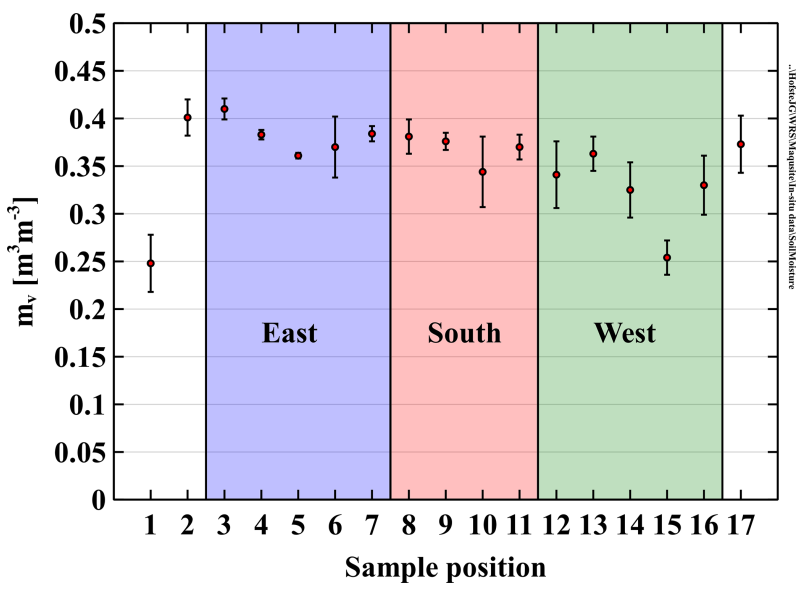

Figure A3. Top soil $m_{\mathrm{V}}$ measured with handheld ThetaProbe at 17 sample positions along the no-step zone periphery (indicated Fig. 2). Vertical bars denote minimum and maximum values of the three measurements per sample position. Red dots represent median values.

\section{A4 Vegetation sampling}

Table A2. Measured vegetation parameters at the Maqu site during summer 2018. Vegetation water content (VWC) is gravimetric: kilogram of water per kilogram of fresh biomass.

\begin{tabular}{lrr}
\hline & 12 July & 17 August \\
& 2018 & 2018 \\
\hline Height (distribution max.) $(\mathrm{cm})$ & 25 & 40 \\
Biomass fresh $\left(\mathrm{kg} \mathrm{m}^{-2}\right)$ & 0.9 & 1.3 \\
Biomass dry $\left(\mathrm{kg} \mathrm{m}^{-2}\right)$ & 0.3 & 0.5 \\
VWC $(\%)$ & 60 & 62 \\
LAI $\left(\mathrm{m}^{2} \mathrm{~m}^{-2}\right)$ & 3.5 & 7 \\
\hline
\end{tabular}




\section{Appendix B: Technical details scatterometer}

\section{B1 Connection scheme and VNA operation}

In Fig. B1 the connection scheme that was used is shown. The front-panel jumpers were removed, and the two dualpolarization broad-band horn antennas were directly connected to the VNA's sources and receivers via the four coaxial cables. This configuration allows for measuring all four polarization channels: vv, vh (i.e. receive in the vertical direction, transmit in the horizontal direction), vh, and hh (Keysight Technologies, 2017). Between all four coaxial cables and their respective VNA connectors, $10 \mathrm{~dB}$ attenuators, type SMA attenuator R411.810.121 (manufacturer: Radiall), were inserted to prevent interference from internal reflections travelling multiple times up and down the coaxial cables.

Measurements were performed by instructing the VNA to measure the four scattering parameters (S parameters) ${ }^{2}(-)$ over a stepped frequency sweep $0.75-10.25 \mathrm{GHz}$. Given the aforementioned connection scheme, the correspondence between recorded $\mathrm{S}$ parameters and transmit/receive polarization channels is as indicated in Fig. B1b. The used connection configuration omits the VNA's internal test-port couplers, which are typically used when measuring (two-port) $\mathrm{S}$ parameters. The VNA software - by default - accounts for these test-port couplers by adding $16 \mathrm{~dB}$ to the signal measured by receivers $\mathrm{A}$ and $\mathrm{B}$ when calculating the $\mathrm{S}$ parameters. With the $\sigma^{0}$ retrieval, this $16 \mathrm{~dB}$ amplification cancels out as the target is divided by the reference return. However, when considering the received powers individually, as done in Sect. 5.2, this factor should be accounted for.

\section{B2 Geometries of experimental setup}

Figure B2a shows all relevant geometries for the performed experiments. The two antenna apertures are at distance $H_{\text {ant }}$ above the ground surface. The separation between the two antenna apertures $W_{\text {ant }}=0.4 \mathrm{~m}$ is small compared to the target distance (ground or calibration standards), which justifies using the geometric centre of the two apertures for all calculations. Every area segment $\mathrm{d} A\left(\mathrm{~m}^{2}\right)$ of the ground surface has its own distance to the antennas $R$ and angle of incidence $\theta$. Angles $\alpha$ and $\beta$ are angular coordinates of $R$. Angle $\alpha$ is defined between the tower's vertical axis and the orthogonal projection of the line from antennas to a ground surface segment onto the plane formed by the tower's vertical axis and the antenna boresight direction line. Angle $\beta$ is defined between the line from antennas to a ground surface segment and projection of that same line onto the plane formed by the tower's vertical axis and the antenna boresight direction line. The planes in which $\alpha$ and $\beta$ lie are also the antenna's principal planes (see for example Balanis, 2005). For the antenna

\footnotetext{
${ }^{2}$ Not to be confused with the scattering amplitudes used in scattering theory, which have units of metres (m); see for example Ulaby and Long (2017).
}

boresight direction $\alpha=\alpha_{0}$ and $\beta=\beta_{0}$. The antenna rotation around the tower's vertical axis is defined as azimuth rotation $\phi$. The green ring on the ground surface in Fig. B2a is related to the time-domain gating process described further on in Sect. B4.

According to Bansal (1999) the antenna's far field distances $R_{\mathrm{ff}}(\mathrm{m})$ are linked to the antenna's largest aperture dimension $D(\mathrm{~m})$ and wavelength $\lambda$ via

$R_{\mathrm{ff}} \geq\left\{\begin{array}{ll}5 D: & \frac{1}{3} \leq \frac{D}{\lambda} \leq \frac{5}{2} \\ \frac{2 D^{2}}{\lambda}: & \frac{5}{2}<\frac{D}{\lambda}\end{array}\right.$.

The antenna aperture is rectangular with dimension $D=$ $0.2 \mathrm{~m}$, which leads to $R_{\mathrm{ff}} \geq 1 \mathrm{~m}$ for $1-3.5 \mathrm{GHz}$ and $R_{\mathrm{ff}} \geq$ $2.7 \mathrm{~m}$ for $3.5-10 \mathrm{GHz}$. Given that with all measurements the distance to the ground surface is larger than $2.7 \mathrm{~m}$, the radiation patterns as measured by the manufacturer apply; see Fig. B3 (Schwarzbeck Mess-Elektronic OHG, 2017).

Figure B2b shows a side view of the setup when radar returns of the reference targets were measured in order to calibrate the scatterometer. The reference targets - a rectangular metal plate and two metal dihedral reflectors - were placed at distances $R_{0}$ from the antennas on top of a metal mast. To shield this mast, pyramidal absorbers were placed in front of it as shown. Next section describes the calibration process in detail.

\section{B3 Calibration}

We measured the radar returns of reference targets with known radar cross section (RCS) $\sigma_{p q}$ in order to calibrate the scatterometer. For the co-polarization channels a rectangular metal plate was used as reference target. As a depolarizing reference target for the cross-polarization channels we used a metal dihedral reflector that was rotated $45^{\circ}$ around the axis perpendicular to the vertex connecting the dihedral's two faces and contained in the symmetry plane also holding the same vertex. The physical optics model used for calculating the RCS of a metal plate and dihedral reflector is

$\sigma_{p p}=4 \pi \frac{(a b)^{2}}{\lambda^{2}}$,

where $a$ and $b$ are the standards' dimensions (m) in the frontal projection (Kerr and Goldstein, 1951). As is shown in for example (Nesti and Hohmann, 1990), Eq. (B2) is also applicable for calculating the cross-polarization RCS of the dihedral reflector when in its rotated position.

There are validity conditions for model (B2) which concern the reference target's size and the distance at which it is measured $R_{0}$. Additionally, the multi-path field illumination of the reference targets (Skolnik, 2008) might be an issue: besides direct illumination from the transmit antenna, radiation reflected from the ground will also illuminate the target; see Fig. B2b. As a result, the direct signal is interfered by these ground-to-target reflections. Table $\mathrm{B} 1$ lists $R_{0}$ values 


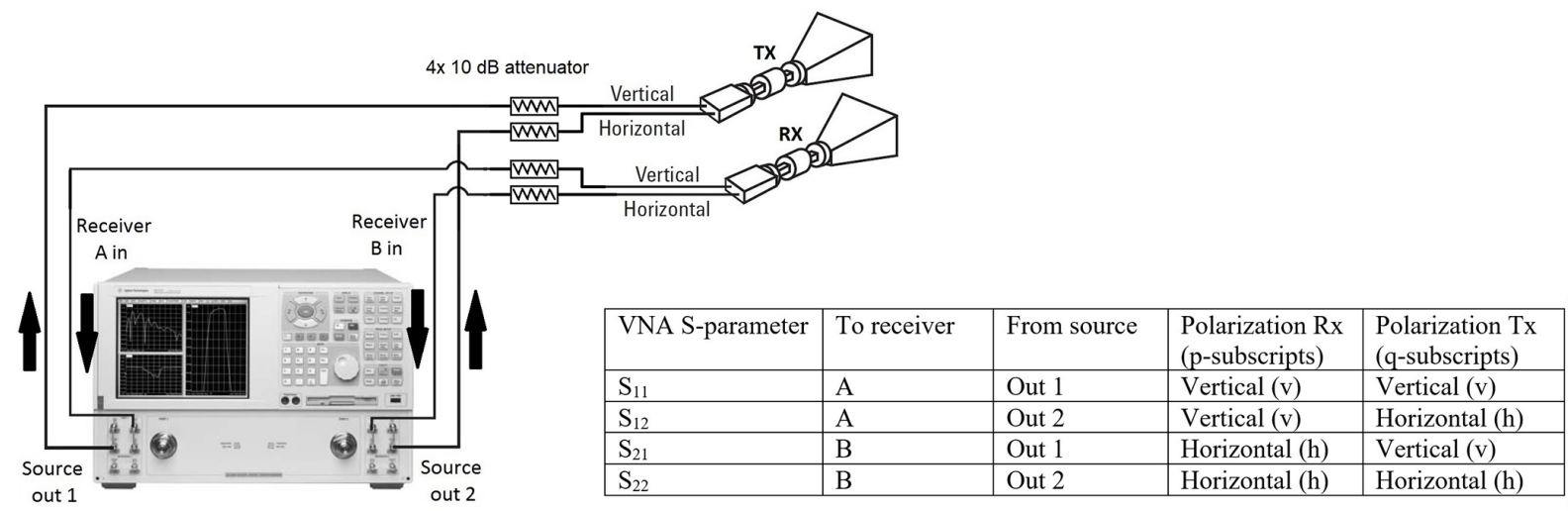

(a)

(b)

Figure B1. Connection scheme of scatterometer and correspondence S parameters to polarization channels for transmit (TX) and receive (RX). (a) Both dual-polarization broad-band antennas, one for TX and the other for RX, are connected to the VNA as indicated (Keysight Technologies, 2017). Arrows indicate direction of signal. (b) Overview correspondence of four VNA S parameters to the four polarization channels.

Table B1. Deployed reference standards and their bandwidths of validity concerning plane wave (PW) and size-to-wavelength criteria.

\begin{tabular}{llll}
\hline & Distance $R_{0}$ & PW criteria met for & $L / \lambda \geq 3$ for \\
\hline Large rectangular plate, $a=85 \mathrm{~cm}, b=65 \mathrm{~cm}$ & $36.3 \mathrm{~m}$ & $f \leq 7.5 \mathrm{GHz}$ & $f \geq 1.5 \mathrm{GHz}$ \\
Small dihedral reflector, $a=57 \mathrm{~cm}, b=38 \mathrm{~cm}$ & $27.7 \mathrm{~m}$ & $f \leq 13 \mathrm{GHz}$ & $f \geq 2.4 \mathrm{GHz}$ \\
Large dihedral reflector, $a=120 \mathrm{~cm}, b=65 \mathrm{~cm}$ & $27.7 \mathrm{~m}$ & $f \leq 3 \mathrm{GHz}$ & $f \geq 1.4 \mathrm{GHz}$ \\
\hline
\end{tabular}

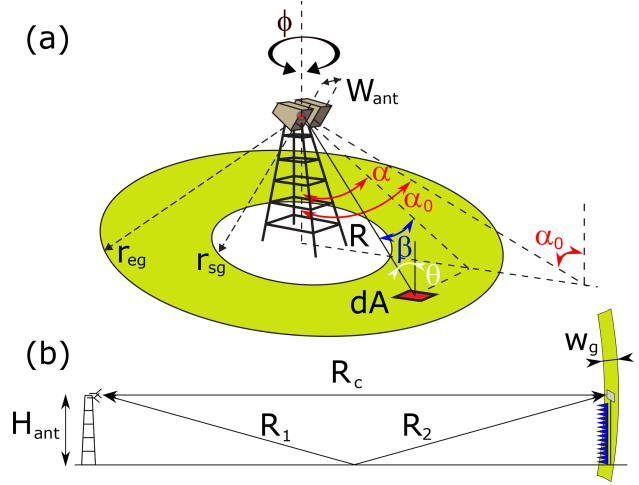

Figure B2. Schematic of scatterometer geometry. (a) Every infinitesimal area $\mathrm{d} A$ has its own distance $R$ to the geometric centre between antenna apertures (red dot) and angle of incidence $\theta$. Angles $\alpha$ and $\beta$ lie within the antennas principal planes, and $\alpha_{0}$ denotes the angle of antenna boresight. The green ring is a projection of the spherical gating shell with radii $r_{\mathrm{sg}}$ and $r_{\text {eg }}$ onto the ground. (b) Side view of geometry during measurement of reference standards. Green ring depicts cross section of spherical gating shell with width $w_{\mathrm{g}}$.

used for the deployed reference standards. We first describe the validity conditions for model (B2).

Conditions for Eq. (B2) are that the standard's largest dimension $L(\mathrm{~m})$ is large compared to the wavelength, i.e. $L>$

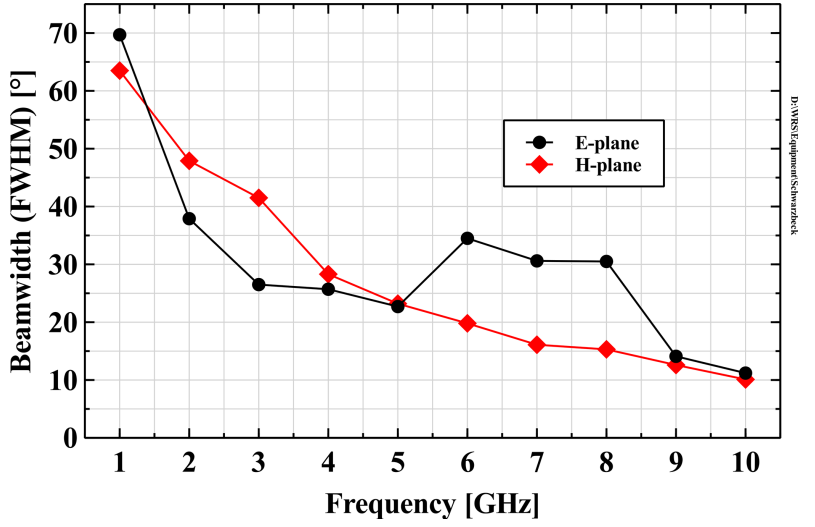

Figure B3. Beamwidths of dual-polarization antennas. Shown is the full width at half maximum (FWHM) of the measured radiation intensity patterns in the two principal planes (Schwarzbeck MessElektronic OHG, 2017).

$\lambda$, and that the incident wavefront is close to planar. Kouyoumjian and Peters (1965) proposed the following equation for calculating the minimum distance $R_{\mathrm{pw}}(\mathrm{m})$ beyond which the wavefront can be considered planar (allowing for a $\pi / 8$ phase error):

$R_{\mathrm{pw}}=\frac{2 L^{2}}{\lambda}$. 
Concerning the condition $L>\lambda$, previous measurements (Hofste et al., 2018) showed, empirically, that for $L / \lambda \geq 3$ model (B2) matches a standard's measured $\sigma_{p p}$ within $1 \mathrm{~dB}$. Besides the $R_{0}$ values used, Table $\mathrm{B} 1$ also lists the frequency ranges for which the plane wave criteria (using the stated values $R_{0}$ ) and the size criteria hold. Strictly speaking, the plane wave criteria with the rectangular plate was not met for 7.5-10 GHz. Yet, the co-polarization $\sigma$ measurement of the small dihedral reflector, discussed in Sect. E3.2, yields results close to the Eq. (B2) value, indicating correct values for $7.5-10 \mathrm{GHz}$.

Now we discuss the possible issue of multi-path illumination by ground-to-target reflections (GTRs). Should the signal strength of these GTRs be significant, the magnitudeover-frequency response of the reference targets will exhibit interference ripples, which complicate interpreting their radar return for the purpose of calibrating the scatterometer. By using gating the GTRs could in principle be removed from the direct target response, provided their difference in geometrical path length is large enough for placing a gating window solely over the direct path reflection in the time domain. The GTR path shown in Fig. B2b was the pathway whose path length was closest to that of the direct route. Also, this GTR path will have the strongest coherent ground reflection since it is specular. Naturally, with smaller $R_{0}$ the difference $R_{0}-\left(R_{1}+R_{2}\right)$ increases, allowing one to better distinguish this GTR from the mean reflection.

However, no (clear) presence of any GTR could be found. Using a $\mathrm{BW}=0.5 \mathrm{GHz}$ bandwidth leads to a $\tau_{\mathrm{p}}=1 / \mathrm{BW}=$ $2 \mathrm{~ns}$ resolution in the time domain, which would allow us to see the shortest GTR-path reflection that - if present - should be at $\left[2 R_{c}-\left(R_{1}+R_{2}+R_{c}\right)\right] / c=5 \mathrm{~ns}$ behind the direct-reflection peak. But even with S-band for hh polarization (broad antenna pattern, and for hh polarization the coherent ground reflection is strongest) no GTR could be found.

Because we could not find evidence of GTR interference we hypothesize that the GTRs were too small in magnitude for our case. The antenna patterns, certainly for the lower frequencies, are broad enough to illuminate a large part of the ground surface, but because of the dense grass cover the coherent forward reflections were probably low. Additionally the bistatic-RCS patterns of both the rectangular plate and dihedral reflector are too narrow, even with L-band, for a sufficient amount of energy to be reflected (in a specular manner) back to the receive antenna. Typically the presence of interference due to multi-path illumination with setups like ours is tested by moving the reference target horizontally over a distance of half a wavelength and observing any changes in the signal. Unfortunately this procedure was not possible with our equipment.

\section{B4 Gating}

For simplicity, instead of using the (complex) electric-field strength measured at the scatterometer's receive antenna $E_{\mathrm{e}}$, we explain the gating process with the term $X(\mathrm{~V})$, which can be considered proportional to $E_{\mathrm{e}}$ by some scatterometer system constant. The measured frequency domain signal $X\left[\omega_{h}\right]$ was transformed into the time domain via the inverse digital Fourier transform (IDFT); see for example Tan and Jiang (2013):

$x\left[t_{n}\right]=\sum_{h=1}^{N} X\left[\omega_{h}\right] e^{i \omega_{h} t_{n}}$.

$N$ is the total number of discrete frequency points within the bandwidth $\mathrm{BW}(\mathrm{Hz})$ considered. Angular-frequency points $\omega_{h}\left(\operatorname{rad~s}^{-1}\right)$ and time points $t_{n}$ (s) are calculated with the minimum and maximum frequency of BW, $f_{\mathrm{lo}}$ and $f_{\text {hi }}$ respectively $(\mathrm{Hz})$, via

$$
\begin{aligned}
\omega_{h} & =2 \pi\left\{[h-1]\left(\frac{f_{\mathrm{hi}}-f_{\mathrm{lo}}}{N-1}\right)+f_{\mathrm{lo}}\right\} \\
h & =1,2,3, \ldots, N \\
t_{n} & =\frac{n-1}{f_{\mathrm{hi}}-f_{\mathrm{lo}}} \quad n=1,2,3, \ldots, N .
\end{aligned}
$$

Next the time-domain response $x\left[t_{n}\right]$ was multiplied by the time-domain filter, or gate, which was a block function of width $\tau_{\mathrm{g}}$ whose sides fell off according to a rapidly decaying Gaussian function, zeroing all signal parts not coinciding with the unity values. The gate's start and end times corresponded to the distances indicated in Fig. B2a: $t_{\mathrm{sg}}=2 r_{\mathrm{sg}} / c$ and $t_{\mathrm{eg}}=2 r_{\mathrm{eg}} / c$ respectively; so in effect, only the surface's scattering events of interest remained in the signal. Graphically, this process is displayed in Fig. B2a. When assuming isotropic radiating and receiving antennas, selecting a certain time gate is equivalent to only considering scattering events within a spherical shell, centred at the antennas, with radii $r_{\mathrm{sg}}$ and $r_{\text {eg }}$. The intersection of said shell with the ground surface then is a ring as shown in the figure. However, our actual antennas have non-isotropic radiation patterns. So they are in fact the surface scattering events associated with the area formed by the intersection of the shown green ring and the scatterometer footprint $A_{\mathrm{fp}}$ that are contained in the signal. As the next step, the gated signal $x\left[t_{n}\right]$ was transformed back into the frequency domain via the digital Fourier transform (DFT):

$X\left[\omega_{h}\right]=\frac{1}{N} \sum_{n=1}^{N} x\left[t_{n}\right] e^{-i \omega_{h} t_{n}}$,

which then contains only the surface scattering information.

The frequency dependence of the radiation patterns, as shown in Fig. B3, complicates the process described above. The time-domain equivalent of the transmitted scatterometer signal is a pulse of width $\tau_{\mathrm{p}}=1 / \mathrm{BW}$ s. Depending on the angle with respect to boresight, i.e. $\alpha$ and $\beta$, this signal pulse will contain different frequencies and will therefore have a 
different temporal shape. At greater angles $\alpha$ and $\beta$, highfrequency components of the pulse are not present, causing the pulse to be broader there. As a result, the footprint area $A_{\text {fp }}$, which is determined from the (known) antenna radiation, or gain patterns $G$ and the gate width $w_{\mathrm{g}}=c \tau_{\mathrm{g}}$ will become broader. We avoided this issue by narrowing our bandwidths such that the radiation patterns of the frequencies within can be considered equal. As a consequence, this meant that for lower frequencies the selected BW had to be more narrow than those for the higher frequencies. The bandwidths used were $1.5-1.75 \mathrm{GHz}$ for L-band, 2.5-3.0 GHz for S-band, for 4.5-5.0 GHz for C-band, and for $9-10 \mathrm{GHz}$ for $\mathrm{X}$-band. Note that there were additional considerations for picking these BW values, which are explained in Sect. C2.

When measuring the reference target backscatter responses $E_{0}\left(\mathrm{~V} \mathrm{~m}^{-1}\right)$, however, the full $0.75-10.25 \mathrm{GHz}$ frequency range can be used. Because the solid angle extending the standard is small we may reasonably assume that all frequencies are present in the time-domain equivalent pulse at the standard, i.e. $G(\alpha, \beta) \approx 1$ for all frequencies. The benefit of using this broad bandwidth $(9.5 \mathrm{GHz})$ is a high temporalspatial resolution in the time domain, which allows for precise placement of the gate over the reference target response.

\section{Appendix C: Technical details $\sigma^{0}$ retrieval procedure}

\section{C1 Implementation of the radar equation}

We rewrite Eq. (3) so that the backscattering coefficient of the surface $\sigma^{0}\left(\mathrm{~m}^{2} \mathrm{~m}^{-2}\right)$ is related to the average received backscattered intensity $\bar{I}\left(\mathrm{Wm}^{-1}\right)$ as (Ulaby and Long, 2017)

$\sigma^{0}=K^{-1} \bar{I}$

where for brevity the polarization subscripts are omitted. The factor $K\left(\mathrm{~W} \mathrm{~m}^{-1}\right)$ is a constant for the bandwidth considered given by

$K=\frac{\lambda^{2}}{4 \pi^{3}} I^{t} \frac{G^{2}}{R_{\mathrm{fp}}^{4}} A_{\mathrm{fp}}$,

where $I^{t}\left(\mathrm{~W} \mathrm{~m}^{-2}\right)$ is the transmitted intensity by the scatterometer. For all terms in $K$ the centre frequency is used. Similar as with Eq. (2), we can substitute $I^{t}$ in Eq. (C2) by the relevant radar parameters when a reference target is measured, yielding

$$
\begin{aligned}
K & =\frac{1}{2} c \epsilon_{0}\left(E_{0}^{\mathrm{g} 0}-E_{\mathrm{b} 0}^{\mathrm{g} 0}-E_{\mathrm{b}}\right)^{2} \frac{G(\alpha, \beta)^{2}}{G\left(\alpha_{0}, \beta_{0}\right)^{2}}\left(\frac{R_{0}}{R_{\mathrm{fp}}}\right)^{4} \frac{A_{\mathrm{fp}}}{\sigma} \\
& =\frac{1}{2} c \epsilon_{0}\left(E_{0}^{\mathrm{g} 0}-E_{\mathrm{b} 0}^{\mathrm{g} 0}-E_{\mathrm{b}}\right)^{2}\left(\frac{R_{0}}{R_{\mathrm{fp}}}\right)^{4} \frac{A_{\mathrm{fp}}}{\sigma} .
\end{aligned}
$$

$E_{0}^{\mathrm{g} 0}\left(\mathrm{~V} \mathrm{~m}^{-1}\right)$ is the measured backscattered field from the reference target (subscript 0 represents "reference"), and $E_{\mathrm{b} 0}^{\mathrm{g} 0}$
$\left(\mathrm{V} \mathrm{m}^{-1}\right)$ is the measured background level during calibration, i.e. the measured backscattered electric field when the calibration standard was removed from the mast while the pyramid absorbers remained in place. With both terms the superscript g0 (for "gate" during reference measurements) indicates that an identical gate was used. The field strength associated with the minimum signal level measurable with the scatterometer is denoted $E_{\mathrm{b}}\left(\mathrm{V} \mathrm{m}^{-1}\right)$. The prefactors light speed $c\left(\mathrm{~ms}^{-1}\right)$ and the permittivity of vacuum $\epsilon_{0}$ $\left(\mathrm{F} \mathrm{m}^{-1} \mathrm{~m}^{-1}\right)$ convert the electric-field strengths into timeaverage intensity. In the middle part of Eq. (C3) the antenna gain functions are written explicitly. $G(\alpha, \beta)$ represents the antenna gain functions when measuring the ground return, while $G\left(\alpha_{0}, \beta_{0}\right)$ represents the situation when the radar return of the reference targets is measured. When using the narrow beam approximation (Eq. 3) and when the reference target is aligned to the antenna boresight direction, the fraction becomes unity and the right part of Eq. (C3) follows. The middle part is used in Appendix Sect. E3.1 when alignment uncertainty of the reference targets is discussed.

In the context of Rayleigh fading statistics with square-law detection (Ulaby et al., 1988), the average received intensity $\bar{I}\left(\mathrm{~W} \mathrm{~m}^{-2}\right)$ is linked to $I_{N}\left(\mathrm{~W} \mathrm{~m}^{-2}\right)$, which is the measured intensity averaged over $N$ independent samples ( $N$ footprints or $N$ frequencies), according to

$\bar{I}=\frac{I_{N}}{1 \pm 1 / \sqrt{N}}$.

Note that $\bar{I}$, like $\sigma^{0}$, is an implied ground surface property. The quantity that is actually measured, $I_{N}$, is an estimator for $\bar{I}$. Equation (C4) holds for $N \geq 10$, since then the probability density function of $I_{N}$ approaches a Gaussian distribution (Ulaby et al., 1982) according to the central limit theorem. The denominator in Eq. (C4) represents a $68 \%$ confidence interval ( \pm 1 standard deviation) for $\bar{I}$. More details on fading are described next in Sect. C2.

In turn, $I_{N}$ is calculated from the measured backscattered electric field from the ground target incident on the receiving antenna $E_{\mathrm{e}}^{\mathrm{g}}\left(\mathrm{V} \mathrm{m}^{-1}\right)$ by

$I_{N}=\frac{1}{2} c \epsilon_{0} \frac{1}{N} \sum_{n=1}^{N}\left(E_{\mathrm{e}}^{\mathrm{g}}\left(f_{n}\right)-\left\langle E_{\mathrm{cr}}^{\mathrm{g}}\right\rangle-E_{\mathrm{b}}\right)^{2}$.

\section{C2 Fading and bandwidth selection}

Fading is the phenomenon where radar return of a distributed target with uniform electromagnetic properties has varying magnitudes and phases when different locations or slightly different frequencies are measured (Ulaby et al., 1988; Monakov et al., 1994). To remove this varying nature from a surface-classifying quantity like $\sigma_{p q}^{0}$, averaging must be performed. By definition $\sigma_{p q}^{0}$ is the average radar cross section of a certain type of distributed target, e.g. forest, asphalt, wheat field, normalized by the illuminated physical surface 
area. $\sigma^{0}$ is proportional to the average measured received power $P^{\mathrm{RX}}$ (Eq. 3) or intensity $\bar{I}$. Therefore, determining $\bar{I}$ and $\sigma^{0}$ requires $N$ statistically independent samples so that the sample average $I_{N}$ approaches the actual average $\bar{I}$ proportionally to $1 / \sqrt{N}$ in accordance with the central limit theorem.

Practically, this can be done either by measuring $I$ at $N$ different locations over the surface, called spatial averaging, or with the frequency averaging technique (see for example Ulaby et al., 1988). With the latter, physical properties governing the scattering, permittivity, and surface roughness are considered frequency invariant over a certain bandwidth. Subsequently, $N$ different frequencies should be selected according to some criteria that account for fading. Both averaging techniques can be used simultaneously as done by Nagarajan et al. (2014) to increase the total number of independent samples. We solely applied the frequency averaging technique because during the time-series measurements our antennas were in a fixed position and orientation. We assumed the single footprint area to be representative for the whole surface of the Maqu site. In Sect. 5.3 we show this assumption is justified. The method used for finding the number $N$ of statistically independent samples within a bandwidth BW is described in Mätzler (1987):

$N=\frac{2 \mathrm{BW} \Delta R}{c}$,

where $\Delta R=r_{\mathrm{sg}}-r_{\mathrm{eg}}$. Subsequently, with $N-1$ intervals of $\Delta f(\mathrm{~Hz}), N$ frequencies are selected from within $\mathrm{BW}$.

As indicated above, with the application of the frequency averaging technique it is assumed that the backscatter behaviour across the selected BW is uniform. To assess the validity of this assumption for bare soil surface, the improved integral equation method $\left(\mathrm{I}^{2} \mathrm{EM}\right)$ surface scattering model (Fung et al., 2002) is applied using the roughness parametrization reported in Dente et al. (2014) and a (frequency-dependent) effective dielectric constant $\epsilon_{\text {soil }}(f)$ according to the dielectric mixing model by Dobson et al. (1985).

Over a BW the mean value $\left\langle\sigma^{0}(\mathrm{BW})\right\rangle$ is calculated, followed by the ratios $\sigma^{0}\left(\mathrm{BW}_{\mathrm{lo}}\right) /\left\langle\sigma^{0}(\mathrm{BW})\right\rangle$ and $\sigma^{0}\left(\mathrm{BW}_{\mathrm{hi}}\right) /\left\langle\sigma^{0}(\mathrm{BW})\right\rangle$ to quantify the change in $\sigma^{0}$ over the $\mathrm{BW}$. In general the $\mathrm{I}^{2} \mathrm{EM}$ model predicts that the change is largest for long wavelengths and smallest for short wavelengths and that it is largest for hh polarization and smallest for vv polarization. Furthermore, the root-mean-square surface height $s(\mathrm{~m})$ is the most sensitive target parameter. As an example, Fig. $\mathrm{C} 1$ shows the calculation result for hh polarization with a BW of $0.5 \mathrm{GHz}$. From the graph we can read that for a centre frequency of $2.75 \mathrm{GHz}$ the retrieved $\sigma_{\mathrm{hh}}^{0}$ for that BW can be expected to vary +1.0 to $-1.2 \mathrm{~dB}$ for $\theta=50^{\circ}$.

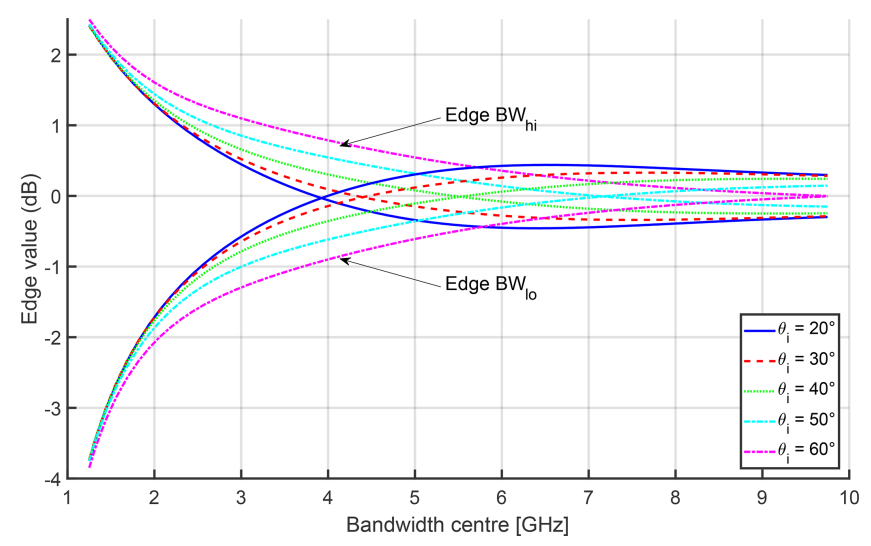

Figure C1. Variation of $\sigma_{\text {hh }}^{0}$ per BW calculated with the combined $\mathrm{I}^{2}$ EM (Fung et al., 2002) and Dobson (Dobson et al., 1985) model. The horizontal axis shows centre frequency of bandwidth $\mathrm{BW}=0.5 \mathrm{GHz}$. Curves indicate the values (in $\mathrm{dB}$ ) to be added to $\left\langle\sigma_{\mathrm{hh}}^{0}(\mathrm{BW})\right\rangle$ at the BW edges for different $\theta$ angles. Shown calculation uses: $s=1 \mathrm{~cm}, \ell=10 \mathrm{~cm}, m_{\mathrm{v}}=0.25 \mathrm{~m}^{3} \mathrm{~m}^{-3}$, and $T_{\text {soil }}=$ $15^{\circ} \mathrm{C}$.

Based on the above calculations we chose $\mathrm{BW}=$ $0.25 \mathrm{GHz}$ for L-band, BW $=0.5 \mathrm{GHz}$ for S- and C-band, and $\mathrm{BW}=1.0 \mathrm{GHz}$ for $\mathrm{X}$-band. These bandwidths will lead to $N$ values around 10, which is sufficient to let the probability density function of $I_{N}$ approach a Gaussian distribution, as explained in Sect. 3.2.3. Further increment of BW was considered not to outweigh the loss of frequency resolution, especially at S-band. 


\section{Appendix D: Three $13 \mathrm{~d}$ time series of $\sigma^{0}$}

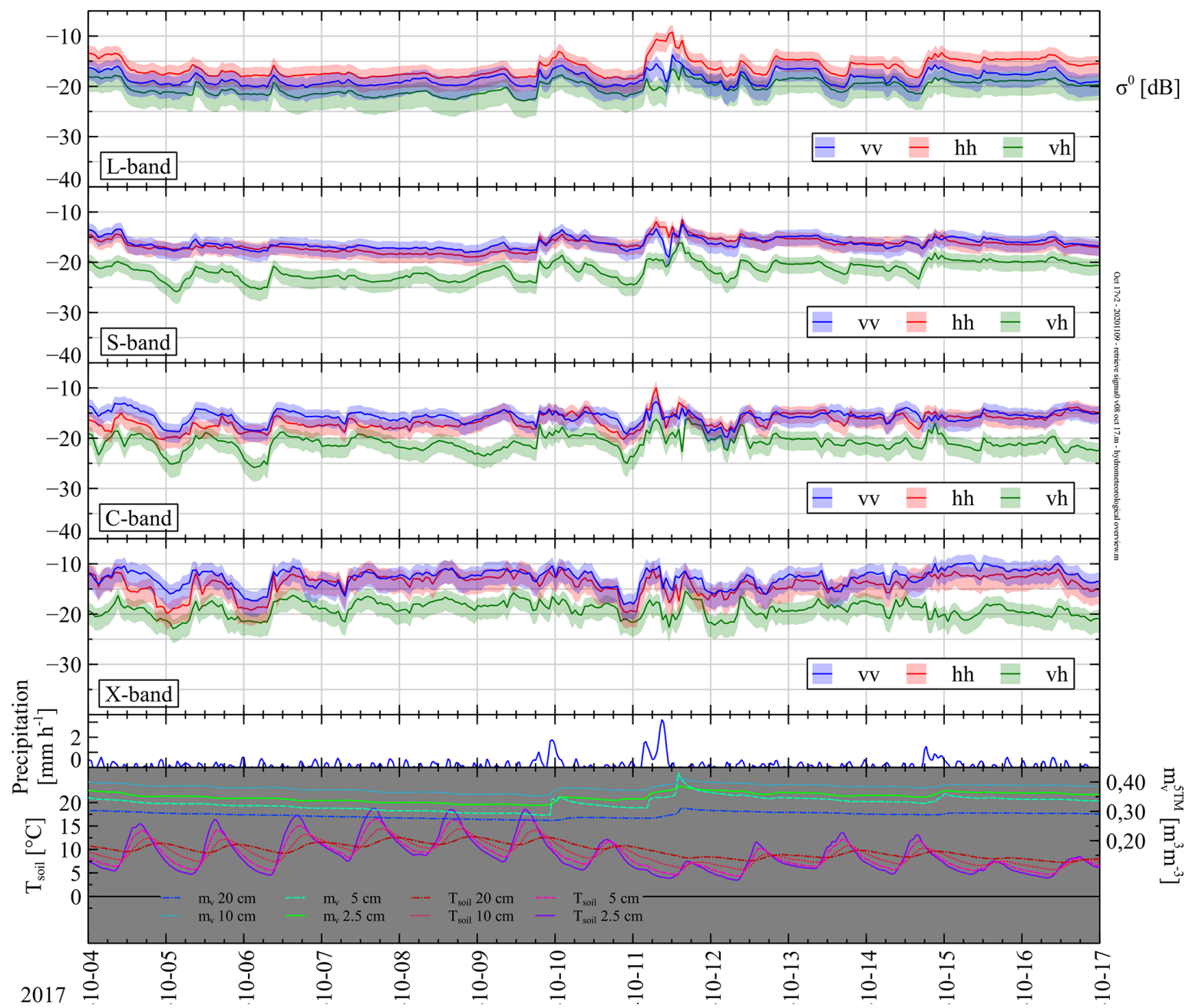

Figure D1. Time-series measurements of $\sigma_{p q}^{0}\left(\mathrm{~m}^{2} \mathrm{~m}^{-2}\right)$ for L-, S-, C-, and X-band, precipitation, $M_{\mathrm{V}}$, and $T_{\text {soil }}$ during $13 \mathrm{~d}$ in October 2017. Shaded regions indicate $66 \%$ confidence intervals for $\sigma_{p q}^{0}$. The antenna boresight angle was fixed at $\alpha_{0}=55^{\circ}$. The incidence angle ranges were band and polarization dependent. The widest ranges were $0^{\circ} \leq \theta \leq 60^{\circ}$ for L-band, $20^{\circ} \leq \theta \leq 60^{\circ}$ for S-band, $36^{\circ} \leq \theta \leq 60^{\circ}$ for $\mathrm{C}$ band, and $47^{\circ} \leq \theta \leq 59^{\circ}$ for X-band. Bottom graphs show measured precipitation $\left(\mathrm{mmh}^{-1}\right)$, volumetric soil moisture $m_{\mathrm{v}}^{5 \mathrm{TM}}\left(\mathrm{m}^{3} \mathrm{~m}^{-3}\right)$, and soil temperature $T_{\text {soil }}$ at indicated depths. Spatial average volumetric soil moisture content $M_{\mathrm{V}}$ is estimated as $M_{\mathrm{V}}=m_{\mathrm{V}}^{5 \mathrm{TM}} \pm 0.04 \mathrm{~m}^{3} \mathrm{~m}^{-3}$. 


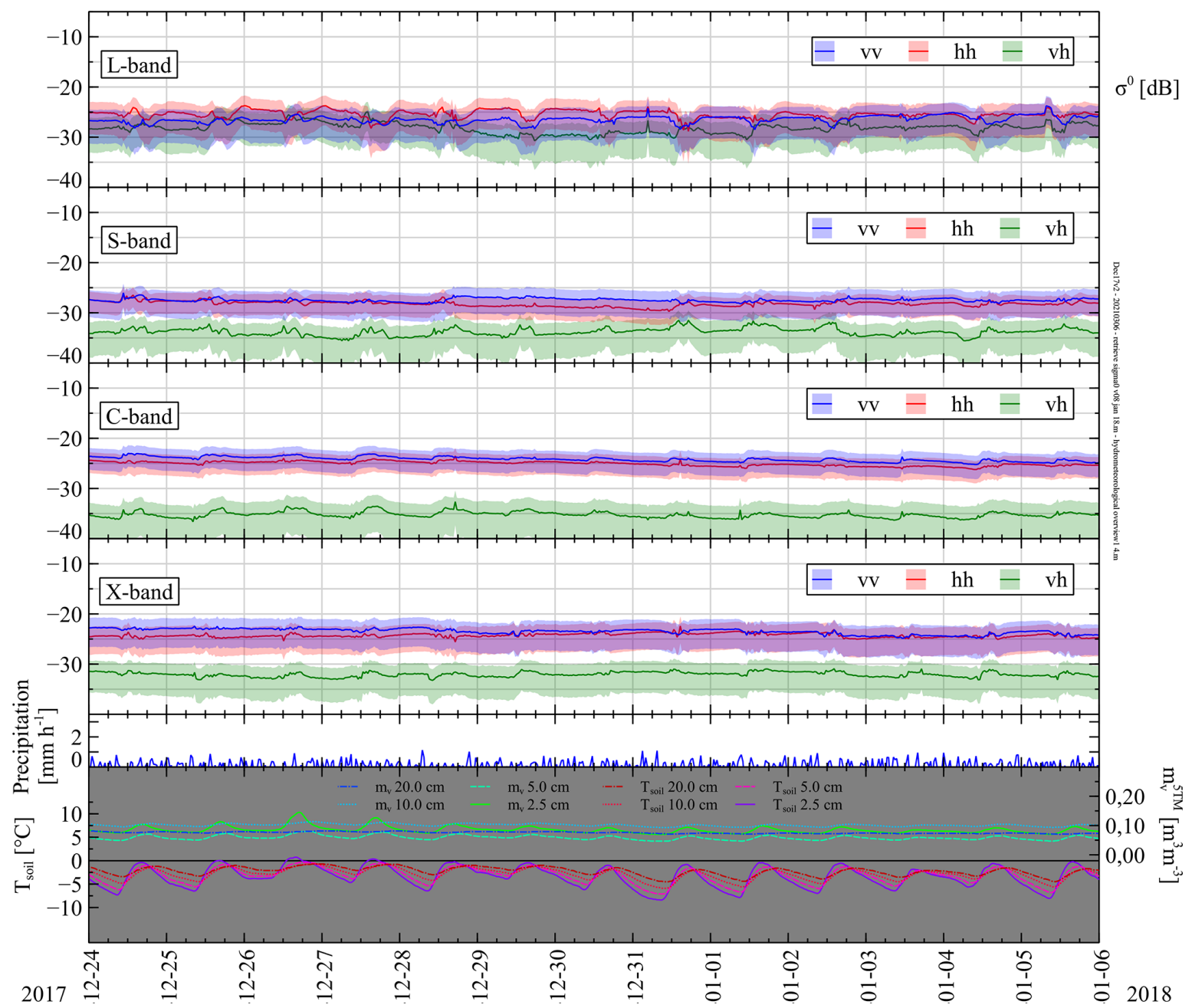

Figure D2. Time-series measurements of $\sigma_{p q}^{0}\left(\mathrm{~m}^{2} \mathrm{~m}^{-2}\right)$ for L-, S-, C-, and X-band, precipitation, $M_{\mathrm{V}}$, and $T_{\text {soil }}$ during $13 \mathrm{~d}$ in December 2017. Same configurations as Fig. D1 apply. 


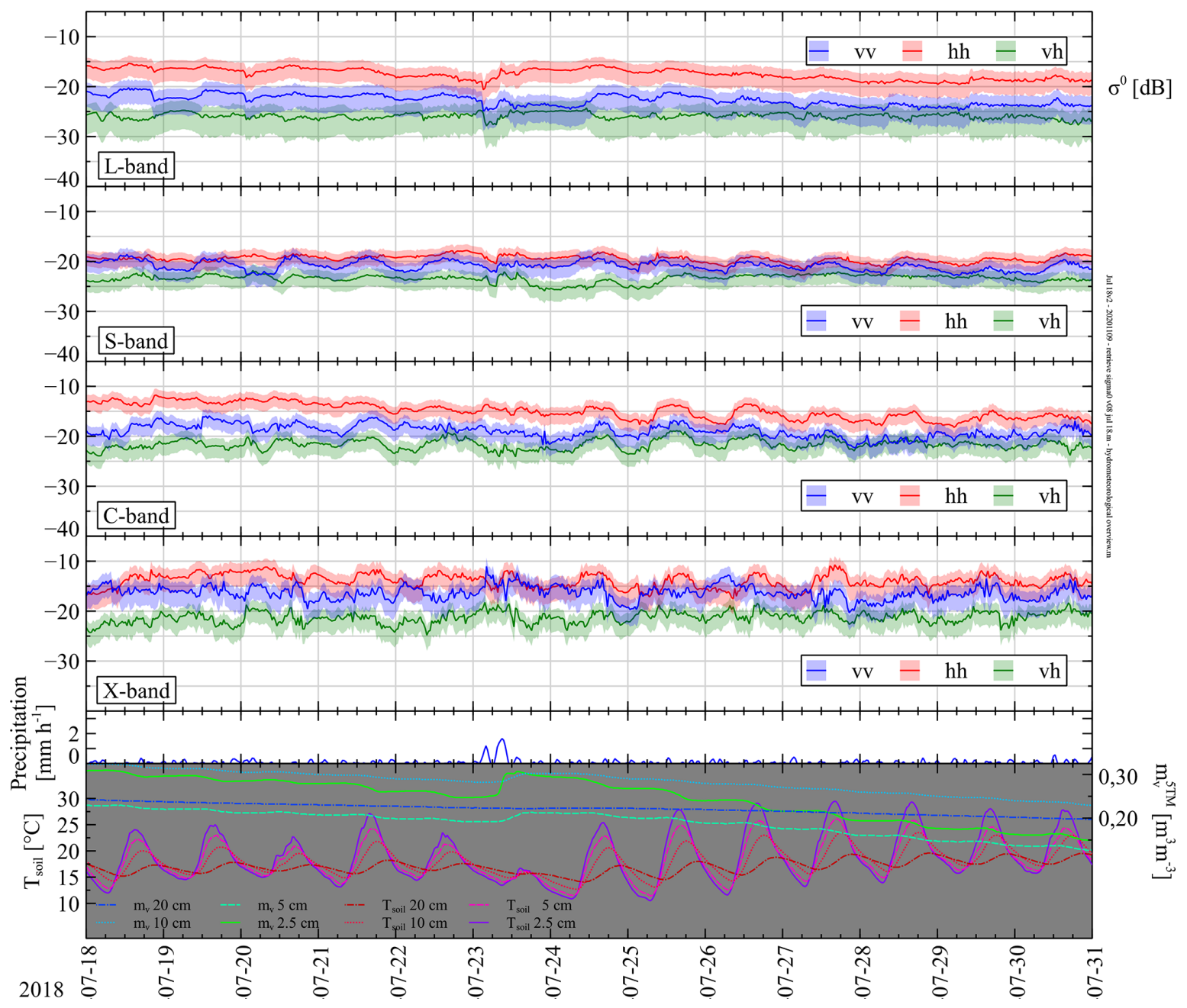

Figure D3. Time-series measurements of $\sigma_{p q}^{0}\left(\mathrm{~m}^{2} \mathrm{~m}^{-2}\right)$ for L-, S-, C-, and X-band, precipitation, $M_{\mathrm{V}}$, and $T_{\text {soil }}$ during $13 \mathrm{~d}$ in July 2018. Same configurations as Fig. D1 apply. 
Appendix E: Details on sources of measurement uncertainty

\section{E1 Overview measurement uncertainty}

Table E1 lists all systematic measurement uncertainties and offsets per BW and polarization channel. The uncertainty $\Delta K$ (Appendix Sect. E3) and $\sigma_{\min }$ values are shown as is, but for the other quantities the resulting receiver power levels (in $\mathrm{dBm}$ ) are shown to allow for comparison with other systems. As explained in Appendix Sect. B1 the VNA actually measures the four $\mathrm{S}$ parameters, which are the (complex) ratios of the received over the transmitted wave voltage for the four polarization channels. The received wave voltages are proportional to the different electric-field strengths $E_{\mathrm{e}}, E_{0}$, etc. described in Sect. 3.2.3. The transmitted wave voltage, or actually its power, is constant at $10 \mathrm{dBm}$ with all measurements. For the calculation of $\sigma^{0}$ by Eq. (C1) it is irrelevant whether the electric-field strengths, wave amplitudes, or S-parameter magnitudes are used since the transmission-related components and/or prefactors simply cancel out. Conversion from measured S parameters (which are associated with the corresponding scattered electric-field strengths) to receiver power is done by subtracting $-16 \mathrm{~dB}$, which was added by the VNA software to account for the test-port coupler, and adding $10 \mathrm{dBm}$. As an example we consider a ground measurement taken on 24 December 2017 at 00:10:00 LT. The VNA measured $\mathrm{d} B\left(S_{11}\right)=-85.24 \mathrm{~dB}$ for $2.8 \mathrm{GHz}$ (S-band) with vv polarization. The power at the VNA receiver then was $-85.24-16+10=-91.24 \mathrm{dBm}$.

From Table E1 we observe that the received power associated with $\Delta E_{T}$ (Appendix Sect. E2) and $E_{\mathrm{cr}}^{\mathrm{g}}$ (Appendix Sect. E4) is, in general, highest for L-band and lowest for Xband. Also, the cross-polarization channels have lower values than those for co-polarization. As for $\Delta E_{T}$, we do not have a clear explanation for this behaviour. For $\left\langle E_{\mathrm{cr}}^{\mathrm{g}}\right\rangle$ we argue that the L-band values are highest due to the stronger coupling because of the broadest radiation patterns at that band. The co-values are higher than with cross polarization because of how the electric-field lines allow for better coupling with the former. The power levels associated with $E_{\mathrm{b}}$ were derived from the specifications documentation of the VNA (Keysight Technologies, 2018). The typical receiver noise levels described therein are specified for a $10 \mathrm{~Hz}$ IF bandwidth. Since we measured with a broader $1 \mathrm{KHz}$ IF bandwidth we added $20 \mathrm{~dB}$ to obtain the values in Table E1. We would like to mention here that the values associated with $\left\langle E_{\mathrm{cr}}^{\mathrm{g}}\right\rangle$ for X-band and the hv channel of C-band were actually lower than the $-120 \mathrm{dBm}$ levels associated with $E_{\mathrm{b}}$. We do not have a clear explanation for this. We therefore consider the $E_{\mathrm{b}}$ as the absolute minimum signal levels and therefore adjusted the values to this level.

The variation of $\sigma_{\min }$ over the bands and polarization channels is due to the variation in measured values of $E_{0}^{\mathrm{g} 0}$. Overall the minimum RCS is about $-50 \mathrm{~m}^{2}(\mathrm{~dB})$. Other studies use the more appropriate so-called noise-equivalent $\sigma^{0}\left(\mathrm{~m}^{2} \mathrm{~m}^{-2}\right)$ to quantify the minimum detectable (distributed) target; see for example Nandan et al. (2016) or Nagarajan et al. (2014). Because of our broad antenna radiation patterns, however, this quantity is not suitable, and therefore we instead refer to a discrete target extending a small solid angle.

\section{E2 Temperature-induced radar return uncertainty}

The performance of the VNA's transmitters and receivers will vary due to variations of their operational temperatures, which in our case are directly linked to the temperature inside the VNA enclosure $T_{\text {encl }}$. Many scatterometer systems employ a so-called internal calibration loop; see for example Ulaby and Long (2017), Baldi (2014), and Werner et al. (2010). This means that beside, or in between, scatterometer measurements, the transmitter and receiver are connected, via a switch, through a reference transmission line of fixed length that has a pre-determined attenuation and phase. This way any fluctuations in the transmitter and/or receiver output over time can be measured and consequentiality removed from the target response. Instead of such an internal calibration loop we employ a different method to account for temperature-induced fluctuations of the VNA's transmitter and receiver performance.

During a half-day time span the antennas were aimed at a fixed target at $21 \mathrm{~m}$ distance: the bare metal mast (without the pyramidal absorbers in front) with a metal sphere on top. At half-hour intervals the radar return was measured together with $T_{\text {encl }}$. The fixed target was assumed to remain constant during that time, so any changes in the radar return were attributed to the changing $T_{\text {encl }}$, which varied from $25-35^{\circ} \mathrm{C}$ during the experiment.

For bandwidths at L-band (1.50-1.75 GHz), S-band (2.53.0 GHz), C-band (4.5-5.0 GHz), and X-band (9.0-9.9 GHz) the radar returns $E_{\mathrm{f}}\left(\mathrm{V} \mathrm{m}^{-1}\right)$ (subscript $\mathrm{f}$ for "fixed target") were filtered by a gate placed over the fixed target timedomain response, resulting in $E_{\mathrm{f}}^{\mathrm{gf}}$ (superscript gf for "gate over fixed target"). The change in $E_{\mathrm{f}}^{\mathrm{gf}}$ over time $t$, and thus over $T_{\text {encl }}$, is denoted $\Delta E_{\mathrm{f}}^{\mathrm{gf}}\left(T_{\text {encl }}\right)$ :

$\Delta E_{\mathrm{f}}^{\mathrm{gf}}\left(T_{\mathrm{encl}}\right)=E_{\mathrm{f}}^{\mathrm{gf}}(t)-E_{\mathrm{f}}^{\mathrm{gf}}(t=0)$.

In Fig. E1 the results of this experiment are shown. Plotted are the bandwidth-average difference of the S-parameter magnitudes over time (and temperature) with respect to the reference value $\Delta S_{\mathrm{f}}^{\mathrm{gf}}\left(T_{\text {encl }}\right)$, alongside $T_{\text {encl }}$. As explained in the main text, the quantities actually measured by the VNA were the S parameters, which are proportional to the corresponding values $E_{\mathrm{f}}^{\mathrm{gf}}$ and $\Delta E_{\mathrm{f}}^{\mathrm{gf}}\left(T_{\mathrm{encl}}\right)$.

There appeared to be no unique relationship between $\Delta S_{\mathrm{f}}^{\mathrm{gf}}$ and $T_{\text {encl }}$. Within $3 \mathrm{~h}$ from the experiment start $T_{\text {encl }}$ increases to a maximum value, after which it decreases again at an increasingly slowed rate. Also the curves $\Delta S_{\mathrm{f}}^{\mathrm{gf}}\left(T_{\mathrm{encl}}\right)$, in gen- 
Table E1. Summary of systematic uncertainties, offsets, and minimum signal levels. Concerning $\Delta E_{T}, E_{\mathrm{cr}}^{\mathrm{g}}$, and $E_{\mathrm{b}}$, table values are the receiver power levels derived from measured $\mathrm{S}$ parameters which, in their turn, are associated with $\Delta E_{T}, E_{\mathrm{cr}}^{\mathrm{g}}$, and $E_{\mathrm{b}}$. With $\Delta K$ and $\sigma_{\text {min }}$ actual values are shown.

\begin{tabular}{|c|c|c|c|c|c|}
\hline & & L-band & S-band & C-band & X-band \\
\hline \multicolumn{6}{|l|}{ Uncertainties } \\
\hline $\begin{array}{l}\text { Temperature-induced radar return uncertainty } \Delta E_{T} \text {. } \\
\mathrm{d} B\left(\Delta S_{T}\right)-16 \mathrm{~dB}+10 \mathrm{dBm}=(\text { in } \mathrm{dBm}) \rightarrow \\
\text { where } \Delta S_{T} \text { is the measured } \mathrm{S} \text { parameter associated } \\
\text { with } \Delta E_{T} \text {. }\end{array}$ & $\begin{array}{l}\text { vv } \\
\text { vh } \\
\text { hv } \\
\text { hh }\end{array}$ & $\begin{array}{r}-95 \\
-107 \\
-103 \\
-98\end{array}$ & $\begin{array}{r}-98 \\
-103 \\
-104 \\
-92\end{array}$ & $\begin{array}{r}-95 \\
-103 \\
-104 \\
-96\end{array}$ & $\begin{array}{l}-103 \\
-104 \\
-103 \\
-103\end{array}$ \\
\hline $\begin{array}{l}\text { Reference target measurement uncertainty } \Delta K \text {. } \\
\quad \text { Relative error (in } \mathrm{dB}) \rightarrow\end{array}$ & $\begin{array}{l}\text { vv } \\
\text { vh } \\
\text { hv } \\
\text { hh }\end{array}$ & $\begin{array}{l} \pm 0.1 \\
\pm 0.4 \\
\pm 0.4 \\
\pm 0.1\end{array}$ & $\begin{array}{l} \pm 0.1 \\
\pm 0.1 \\
\pm 0.1 \\
\pm 0.1\end{array}$ & $\begin{array}{l} \pm 0.2 \\
\pm 0.2 \\
\pm 0.2 \\
\pm 0.3\end{array}$ & $\begin{array}{l} \pm 1.0 \\
\pm 0.8 \\
\pm 0.8 \\
\pm 1.0\end{array}$ \\
\hline \multicolumn{6}{|l|}{ Offsets and minimum signal levels } \\
\hline $\begin{array}{l}\text { Offset due to antenna coupling remnant } E_{\mathrm{cr}}^{\mathrm{g}} \text {. } \\
\qquad \mathrm{d} B\left(\left\langle S_{\mathrm{cr}}^{\mathrm{g}}\right\rangle\right)-16 \mathrm{~dB}+10 \mathrm{dBm}=(\text { in } \mathrm{dBm}) \rightarrow \\
\text { where }\left\langle S_{\mathrm{cr}}^{\mathrm{g}}\right\rangle \text { is the measured } \mathrm{S} \text { parameter, averaged } \\
\text { over } \mathrm{BW} \text {, associated with } E_{\mathrm{cr}}^{\mathrm{g}} \text {. }\end{array}$ & $\begin{array}{l}\text { vv } \\
\text { vh } \\
\text { hv } \\
\text { hh }\end{array}$ & $\begin{array}{l}-86 \\
-92 \\
-96 \\
-82\end{array}$ & $\begin{array}{r}-103 \\
-102 \\
-104 \\
-91 \\
\end{array}$ & $\begin{array}{l}-113 \\
-119 \\
-120 \\
-107\end{array}$ & $\begin{array}{l}-120 \\
-120 \\
-120 \\
-120\end{array}$ \\
\hline $\begin{array}{l}\text { Minimum detectable signal level } E_{\mathrm{b}} \text {. } \\
\mathrm{d} B\left(S_{\mathrm{b}}\right)-16 \mathrm{~dB}+10 \mathrm{dBm}=(\text { in } \mathrm{dBm}) \rightarrow \\
\text { where } S_{\mathrm{b}} \text { is the measured } \mathrm{S} \text { parameter, averaged } \\
\text { over } \mathrm{BW} \text {, associated with } E_{\mathrm{b}} \text {. }\end{array}$ & & -119 & -120 & -120 & -120 \\
\hline $\begin{array}{l}\text { Minimum detectable RCS value } \sigma_{\mathrm{min}} \text {. } \\
\quad \text { Given target distance is } R_{\mathrm{fp}}\left(\mathrm{m}^{2} \text { expressed in } \mathrm{dB}\right) \rightarrow\end{array}$ & $\begin{array}{l}\text { vv } \\
\text { vh } \\
\text { hv } \\
\text { hh }\end{array}$ & $\begin{array}{l}-53 \\
-49 \\
-50 \\
-53\end{array}$ & $\begin{array}{l}-52 \\
-51 \\
-52 \\
-54\end{array}$ & $\begin{array}{l}-51 \\
-51 \\
-51 \\
-52\end{array}$ & $\begin{array}{l}-48 \\
-49 \\
-51 \\
-50\end{array}$ \\
\hline
\end{tabular}

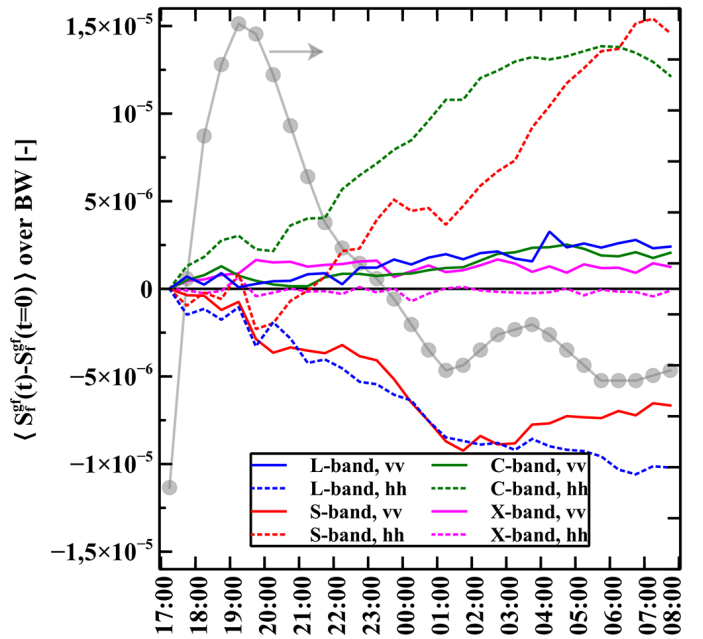

(a)
Time

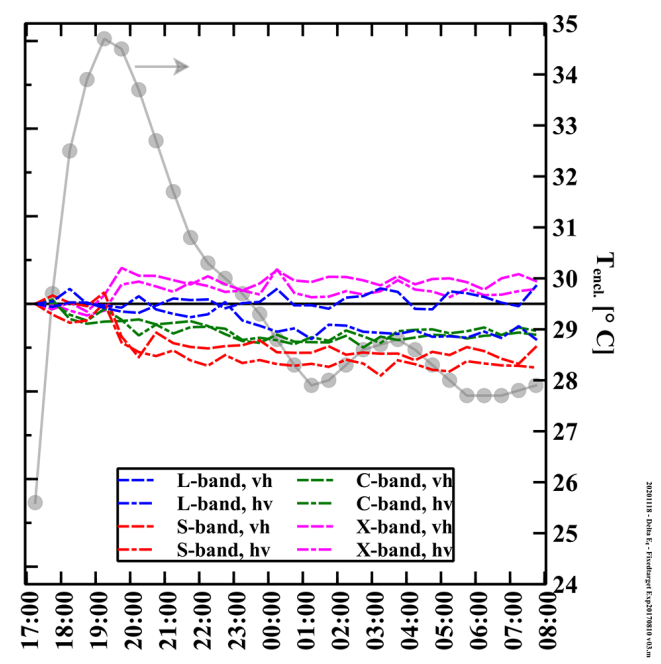

Time

(b)

Figure E1. Measured radar return from a fixed target over a varying enclosure temperature $T_{\text {encl }}$. Shown times are in local time. 
eral, change more rapidly over the first $5 \mathrm{~h}$ and then become more stable. However, the direction of change in $T_{\text {encl }}-$ a rapid increase at the start followed by a fading decrease after 19:15 LT - is not seen in the $\Delta S_{\mathrm{f}}^{\mathrm{gf}}\left(T_{\text {encl }}\right)$ curves. So in order to quantify the temperature-induced VNA instability we used the maximum observed variation of $\Delta S_{\mathrm{f}}^{\mathrm{gf}}\left(T_{\mathrm{encl}}\right)$ over time amidst all frequencies within $\mathrm{BW}$ to calculate the temperature-induced radar return uncertainty $\Delta S_{T}$ or, in the context of scattered electric-field strengths, its corresponding value $\Delta E_{T}\left(\mathrm{~V} \mathrm{~m}^{-1}\right)$ :

$$
\Delta E_{T}=\frac{\max \left[\Delta E_{\mathrm{f}}^{\mathrm{gf}}\left(T_{\mathrm{encl}}\right)\right]-\min \left[\Delta E_{\mathrm{f}}^{\mathrm{gf}}\left(T_{\mathrm{encl}}\right)\right]}{2} .
$$

The quantity $\Delta E_{T}$ is to be treated as an absolute uncertainty of $E_{\mathrm{e}}^{\mathrm{g}}$ (Eq. C5) according to

$$
I_{N}=\frac{1}{2} c \epsilon_{0} \frac{1}{N} \sum_{n=1}^{N}\left(E_{\mathrm{e}}^{\mathrm{g}}\left(f_{n}\right)-\left\langle E_{\mathrm{cr}}^{\mathrm{g}}\right\rangle-E_{\mathrm{b}} \pm 2 \Delta E_{T}\right)^{2},
$$

with a factor two since both $E_{\mathrm{e}}^{\mathrm{g}}\left(f_{i}\right)$ and $E_{\mathrm{b}}^{\mathrm{g}}\left(f_{n}\right)$ are subject to this temperature-induced uncertainty. Table E1 lists the power levels at the VNA's receivers calculated from $\Delta S_{T}$ for the considered bandwidths and polarization channels.

\section{E3 Reference target measurement uncertainty}

\section{E3.1 Reference target alignment}

The absolute backscattering coefficient is determined with respect to the known RCS of a reference target. Errors in the used reference target RCS itself or errors made during the measurement of that target will contribute to the $\sigma^{0}$ uncertainty. The RCS of a rectangular metal plate calculated with Eq. (B2) was found to match experimental observations fairly well (Ross, 1966), and therefore errors in the RCS of our rectangular plate itself were not considered. For the dihedral reflector we did the same, keeping in mind to only gate the specular reflection in the time domain and not also include the dihedral's edge reflections. As shown in Sorensen (1991) interference from diffraction of the dihedral's edges is not accounted for in Eq. (B2). We did consider errors in the measurement of the reference target; specifically we considered misalignment of the scatterometer's antennas towards the rectangular plate and vice versa.

The angular position of the reference targets with respect to the antenna boresight direction was estimated to be $-2.25^{\circ} \leq \beta_{0} \leq 1.25^{\circ}$ in the horizontal direction and $-1.3^{\circ} \leq \alpha_{0} \leq 1.3^{\circ}$ in the vertical direction. Given the large distance from the antennas to the rectangular plate, $R_{0}=$ $36.3 \mathrm{~m}$, and the much smaller separation between the transmit and receive antennas, $W_{\text {ant }}=0.4 \mathrm{~m}$, single uncertainty values $\Delta \alpha_{0}$ and $\Delta \beta_{0}$ were used for both antennas. Due to this possible antenna misalignment the reference target is not illuminated by the peak value of the gain pattern, i.e.
$G=G\left(\alpha_{0} \pm \Delta \alpha_{0}, \beta_{0} \pm \Delta \beta_{0}\right)(-)$, resulting in an uncertainty in the measured radar response of the reference target and thus in $K\left(\mathrm{~W} \mathrm{~m}^{-1}\right)$. Equation (C3) then is modified to

$$
\begin{aligned}
K & =\frac{1}{2} c \epsilon_{0}\left(E_{0}^{\mathrm{g} 0}-E_{\mathrm{b} 0}^{\mathrm{g} 0}-E_{\mathrm{b}}\right)^{2} \\
& \times \frac{G(\alpha, \beta)^{2}}{G\left(\alpha_{0} \pm \Delta \alpha_{0}, \beta_{0} \pm \Delta \beta_{0}\right)^{2}}\left(\frac{R_{0}}{R_{\mathrm{fp}}}\right)^{4} \\
& \times \frac{A_{\mathrm{fp}}}{\sigma^{\mathrm{bi}}\left(\theta_{\mathrm{i}} \pm \Delta \theta_{\mathrm{i}}, \phi_{\mathrm{i}}, \theta_{\mathrm{s}} \pm \Delta \theta_{\mathrm{s}}, \phi_{\mathrm{s}}\right)} .
\end{aligned}
$$

The angular position of the individual antennas with respect to the reference target's surface normal (or frontal projection surface normal in case of the dihedral reflectors) was estimated with the help of a laser mounted between the two antennas and detachable mirrors on the reference targets. Optimal alignment was found by rotating the targets until the reflected laser spot was on (or close to) the laser aperture again. In the horizontal plane, the angle between the rectangular plate's surface normal and the transmit antenna was $\theta_{\mathrm{i}}=$ $0.16^{\circ}$ (right side of the normal) and for the receive antenna $\theta_{\mathrm{s}}=-0.48^{\circ}$. In the vertical plane, the angle between the rectangular plate's surface normal and both antennas (as they are next to each other) was close to zero. We estimated the uncertainty of all aforementioned angles to be $\Delta \theta_{\mathrm{i}}=\Delta \theta_{\mathrm{s}}=0.10^{\circ}$ (both in the horizontal and vertical plane). For the small dihedral reflector these angles were $\theta_{\mathrm{i}}=\theta_{\mathrm{s}}=0 \pm 0.2^{\circ}$ in the horizontal and vertical plane, while for the large dihedral reflector $\theta_{\mathrm{i}}=1.34 \pm 0.2^{\circ}$ and $\theta_{\mathrm{s}}=0.52 \pm 0.2^{\circ}$ in the horizontal plane and $\theta_{\mathrm{i}}=\theta_{\mathrm{s}}=0.72 \pm 0.2^{\circ}$ in the vertical plane.

Starting with the physical optics model for the monostatic RCS of a metal rectangular plate, $\sigma(\theta, \phi)$ (Kerr and Goldstein, 1951, p. 457), a crude bistatic-RCS version $\sigma^{\mathrm{bi}}\left(\theta_{\mathrm{i}}, \phi_{\mathrm{i}}, \theta_{\mathrm{S}}, \phi_{\mathrm{S}}\right)$ was created by simply imposing a linear phase delay along the plate's surface. We shall assume that this model will also hold for the dihedral reflector. Calculation of $K$ can then be extended to include the (mis)alignment or offset of both individual antennas with respect to the reference targets and their uncertainties, which leads to Eq. (E4).

How the uncertainties $\Delta \alpha_{0}, \Delta \beta_{0}, \Delta \theta_{\mathrm{i}}$, and $\Delta \theta_{\mathrm{s}}$ in Eq. (E4) propagate into the uncertainty of $K$, called the reference target measurement uncertainty $\Delta K$, may be found in textbooks such as Hughes and Hase (2010). Resulting $\Delta K$ values, per considered $\mathrm{BW}$ and polarization, are presented as relative uncertainties in Table E1. With X-band the $\Delta K$ values are highest because the antenna radiation patterns are most narrow for higher frequencies.

\section{E3.2 Validation reference target alignment}

In this section we shall demonstrate that estimated values for the rotational offsets and uncertainties $\theta_{\mathrm{i}}, \theta_{\mathrm{s}}, \Delta \theta_{\mathrm{i}}$, and $\Delta \theta_{\mathrm{s}}$ of the reference targets used are consistent with their respective measured radar returns. First we apply the radar equation (Eq. 1) to both the rectangular plate and the small dihedral 
reflector and substitute for $P^{\mathrm{TX}}$. We then have

$$
\begin{aligned}
\sigma_{\mathrm{dih}}^{\mathrm{bi}}\left(\theta_{\mathrm{i}}^{\mathrm{dih}}, \phi_{\mathrm{i}}, \theta_{\mathrm{s}}^{\mathrm{dih}}, \phi_{\mathrm{s}}\right) & =\frac{P_{\mathrm{dih}}^{\mathrm{RX}}}{P_{\mathrm{pla}}^{\mathrm{RX}}} \frac{G\left(\alpha_{0} \pm \Delta \alpha_{0}, \beta_{0} \pm \Delta \beta_{0}\right)^{2}}{G\left(\alpha_{0} \pm \Delta \alpha_{0}, \beta_{0} \pm \Delta \beta_{0}\right)^{2}} \\
& \times\left(\frac{R_{\mathrm{dih}}}{R_{\text {pla }}}\right)^{4} \sigma_{\text {pla }}^{\mathrm{bi}}\left(\theta_{\mathrm{i}}^{\text {pla }}, \phi_{\mathrm{i}}, \theta_{\mathrm{s}}^{\mathrm{pla}}, \phi_{\mathrm{s}}\right),
\end{aligned}
$$

where we dropped the polarization subscripts for readability. Since the values for $\alpha_{0}$ and $\beta_{0}$ are the same for both measurements, the term containing the antenna gain patterns $G$ is unity. We then end up with

$$
\frac{\sigma_{\text {dih }}^{\mathrm{bi}}\left(\theta_{\mathrm{i}}^{\mathrm{dih}}, \phi_{\mathrm{i}}, \theta_{\mathrm{s}}^{\mathrm{dih}}, \phi_{\mathrm{s}}\right)}{\sigma_{\mathrm{pla}}^{\mathrm{bi}}\left(\theta_{\mathrm{i}}^{\mathrm{pla}}, \phi_{\mathrm{i}}, \theta_{\mathrm{s}}^{\mathrm{pla}}, \phi_{\mathrm{s}}\right)}=\left(\frac{R_{\mathrm{dih}}}{R_{\mathrm{pla}}}\right)^{4} \frac{P_{\mathrm{dih}}^{\mathrm{RX}}}{P_{\mathrm{pla}}^{\mathrm{RX}}} .
$$

Figure E2 shows the measured radar returns of the three calibration standards. For $5 \mathrm{GHz}$ the difference between the small dihedral return $P_{\mathrm{dih}}^{\mathrm{RX}}$ and the rectangular plate $P_{\text {rect }}^{\mathrm{RX}}$ for $\mathrm{vv}$ polarization is $-3.3 \mathrm{~dB}$. The term involving the distances $R$ is $-4.7 \mathrm{~dB}$, resulting in the right side of Eq. (E6) being $-8.0 \mathrm{~dB}$. If both reference targets were perfectly aligned towards the antennas, the RCS ratio on the left side of Eq. (E6) would be $-8.1 \mathrm{~dB}$, which is $0.1 \mathrm{~dB}$ below the measured result. By finding suitable combinations of misalignment or offset angles $\theta_{\mathrm{i}}$ and $\theta_{\mathrm{s}}$ for both targets, Eq. (E6) can be satisfied. It can be shown that consistent angles can be found for all three reference targets which are within the ranges specified in Sect. E3.1. In the above procedure we used the copolarization returns of the dihedral reflectors, while it is in fact the cross polarization that is of interest. We argue that the $45^{\circ}$ rotation of the dihedral references - necessary for realizing the depolarization - does not introduce other significant angular offsets, and therefore the estimated values for the rotational offsets and uncertainties remain valid also for cross polarization. Note that the explained method cannot validate the angular positions of the reference targets with respect to the antenna boresight direction and their uncertainties $-\alpha_{0}$, $\Delta \alpha_{0}$ and $\beta_{0}, \Delta \beta_{0}-$ as the term containing the antenna gain patterns was cancelled out.

We conclude this section with some remarks on the features in the measured reference target return powers shown in Fig. E2. With all returns there is a sharp trough between 8-9 GHz, which is caused by a combination of a local increment of the antenna's return loss and an asymmetry in the antennas' E-plane radiation pattern between 7-9 GHz. The asymmetry causes the pattern's peaks to point off-target by about $10^{\circ}$, resulting in a lower radar return. The deep troughs close to $1.3 \mathrm{GHz}$ are caused by a combination of high return loss at the low-frequency edge of the antenna's operational bandwidth and an artefact of the gating procedure, which in this case lets $E_{0}^{\mathrm{g} 0}(f)$ rise at the edge. This gating artefact is known to distort the band edges of a gated frequency response (Keysight Technologies, 2020). To account for this artefact the bandwidths used for the ground surface measure-

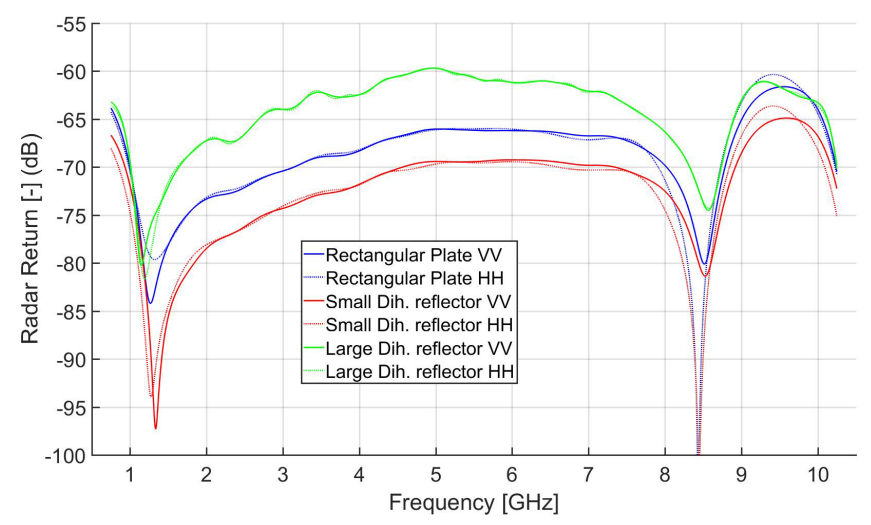

Figure E2. Measured radar returns of calibration standards for copolarization $E_{0}^{\mathrm{g} 0}$. Solid lines are $\mathrm{VV}$, and dotted lines are $\mathrm{HH}$ polarization.

ments were broadened by $10 \%$ at both edges prior to gating. The added edges were discarded again after gating. The curves of the rectangular plate and small dihedral reflector have a similar shape for most of the frequency band. Their difference is merely a constant factor as predicted by the physical optics model for RCS (Eq. B2). The curve shape of the large dihedral reflector however is clearly different from the other two. This is partly because of its more severe angular offsets $\theta_{\mathrm{i}}$ and $\theta_{\mathrm{s}}$ but also because the planar-wave condition is not met for most of the frequency band; see Table B1.

\section{E4 Antenna coupling remnant}

Because the transmit and receive antennas are placed next to each other in order to measure the monostatic $\sigma^{0}$, part of the transmitted signal leaks, or couples, directly into the receive antenna, thereby interfering with the target return of interest. This antenna coupling is strongest for the lower frequencies (L-band) because these have the broadest antenna radiation patterns (see Fig. B3). With respect to the polarization channels, the antenna coupling is strongest for hh because of how the electric-field lines of the principal $\mathrm{TE}_{10}$ modes, in the particular case of hh polarization, couple strongest when the antenna apertures are next to each other. With the crosspolarization channels the coupling is weakest because of how the principal field components are perpendicular between the transmit and receive antenna.

Although the majority of the antenna coupling can be filtered out by gating, a remnant remains present in the filtered frequency domain response. This becomes apparent when the antennas are pointed skywards and the time-domain response is calculated per BW. Between the times and distances $r_{\mathrm{sg}}=c t_{\mathrm{sg}} / 2$ and $r_{\mathrm{eg}}=c t_{\mathrm{eg}} / 2$, where, during measurement of the ground target, the scattering events of interest are located, the signal is not yet at its lowest level beyond $10 \mathrm{~m}$. This effect is strongest for the L-band BW with hh po- 
larization, while for $\mathrm{X}$-band the time-domain response level between $r_{\text {sg }}$ and $r_{\text {eg }}$ is almost equal to its lowest level.

From the sky measurement the coupling remnant $E_{\mathrm{cr}}^{\mathrm{g}}(\mathrm{BW})$ was retrieved. When measuring the ground surface, the antenna coupling process of course interferes with the ground return. However, because we measure over a bandwidth and the ground return is a randomly fluctuating signal, we argue that $\left\langle E_{\mathrm{cr}}^{\mathrm{g}}(\mathrm{BW})\right\rangle$, so the average over BW can simply be subtracted from the (gated) ground return $E_{\mathrm{e}}^{\mathrm{g}}(\mathrm{BW})$.

\section{E5 Propagation of uncertainties}

In this section we demonstrate how Eq. (4) is derived. We show, using error propagation theory, how each of the (three) error terms, $\Delta E_{T}, \Delta K$, and fading, propagates into an error for $\sigma^{0}$ and how all errors may be combined into one statistical confidence interval for $\sigma^{0}$. We start with Eq. (C1), which with Eq. (C4) can be written as

$\sigma^{0}=\frac{\bar{I}}{K}=\frac{I_{N}}{K(1 \pm 1 / \sqrt{N})}$.

The term between brackets in the denominator we may simply rewrite as $F \pm \Delta F$, i.e. a variable with an error. The variables $I_{N}$ and $K$ also have their respective errors $\Delta I_{N}$ and $\Delta K$. When we write all variables and their errors explicitly we end up with

$\sigma^{0}=\frac{I_{N}}{K F}=\frac{I_{N} \pm \Delta I_{N}}{(K \pm \Delta K)(F \pm \Delta F)}$.

We shall now describe all three error terms, starting with $\Delta I_{N}$. The calculation of $I_{N}$ from the measured backscattered electric field is given by Eq. (E3) as

$I_{N}=\frac{1}{2} c \epsilon_{0} \frac{1}{N} \sum_{n=1}^{N}\left(E_{\mathrm{e}}^{\mathrm{g}}\left(f_{n}\right)-\left\langle E_{\mathrm{cr}}^{\mathrm{g}}\right\rangle-E_{\mathrm{b}} \pm 2 \Delta E_{T}\right)^{2}$,

with $\Delta E_{T}$ as measurement uncertainty. As explained in Sect. C2, every term in the above sum may be considered an independent variable. Because the number of samples $N$ within BW is sufficiently large (about 15), we consider $\Delta E_{T}$ as a statistical error and therefore use the corresponding equation for error propagation (see for example Hughes and Hase, 2010) to calculate the total statistical error $\Delta I_{N}$ :

$\Delta I_{N}=\frac{1}{2} c \epsilon_{0} \frac{4 \Delta E_{T}}{N} \sqrt{\sum_{n=1}^{N}\left(E_{\mathrm{e}}^{\mathrm{g}}\left(f_{n}\right)-\left\langle E_{\mathrm{cr}}^{\mathrm{g}}\right\rangle-E_{\mathrm{b}}\right)^{2}}$.

$\Delta I_{N}$ can be considered the 1-standard-deviation value of $I_{N}$. Since the number of terms in the sum $N$ are large enough, we can consider $\pm \Delta I_{N}$ as the edges of a $66 \%$ confidence interval for $I_{N}$.

As explained in Sect. E3.1, $\Delta K$ can be calculated by using error propagation theory for the errors $\Delta \alpha_{0}, \Delta \beta_{0}$, and those associated with the bistatic RCS of the rectangular metal plate and dihedral reflectors $\Delta \theta_{\mathrm{i}}$ and $\Delta \theta_{\mathrm{s}}$. Note, however, that these are maximum possible errors so that the corresponding error propagation rules should be used. In order to have differentiable functions for the E-plane and H-plane antenna gain patterns, $E_{\text {patt }}\left(\alpha_{0}\right)$ and $H_{\text {patt }}\left(\beta_{0}\right)$ respectively, the measured radiation patterns can be fitted with Gaussian functions for angles close to antenna boresight. Writing $\Delta K$ explicitly is then straightforward.

Finally, we have the error term $\Delta F$, which is equal to $1 / \sqrt{N}$. As explained in Sect. C1 this error represents a $68 \%$ confidence interval for $\bar{I}$.

Returning to Eq. (E8) we now combine all three errors into one statistical error. To do so we must first convert $\Delta K$ from being a maximum possible error into a statistical error like $\Delta I_{N}$ and $\Delta F$. This can be done by multiplying $\Delta K$ with $2 / 3$, so the result may be interpreted as a 1 -standard-deviation value for $K$. This is equivalent to saying that $\pm 2 / 3 \Delta K$ is a $68 \%$ confidence interval for $K$. We combine the three statistical errors conservatively into a $66 \%$ confidence interval for $\sigma^{0}$ :

$$
\begin{aligned}
\sigma^{0} & =\frac{I_{N}}{K F}=\frac{I_{N} \pm \Delta I_{N}}{\left(K \pm \frac{2}{3} \Delta K\right)(1 \pm 1 / \sqrt{N})} \\
& =\frac{I_{N}}{K F} \pm \Delta \sigma^{0}=\frac{I_{N}}{K} \pm \Delta \sigma^{0},
\end{aligned}
$$

where $\Delta \sigma^{0}$ is calculated according to the error propagation equation for statistical errors:

$$
\begin{aligned}
\left(\Delta \sigma^{0}\right)^{2} & =\left(\frac{\partial \sigma^{0}}{\partial I_{N}}\right)^{2}\left(\Delta I_{N}\right)^{2}+\left(\frac{\partial \sigma^{0}}{\partial K}\right)^{2}(\Delta K)^{2} \\
& +\left(\frac{\partial \sigma^{0}}{\partial F}\right)^{2}(\Delta F)^{2}
\end{aligned}
$$

\section{Appendix F: Angular variation of $\sigma_{p p}^{0}$ for asphalt}

We start with the asphalt experiment results, which we present here to demonstrate that our $\sigma^{0}$ retrieval method, using measurement data obtained with our scatterometer system, results in $\sigma^{0}$ values comparable to those in other studies.

Figure F1 shows our retrieved $\sigma_{p q}^{0}$ over $\alpha_{0}$ for all bandwidths and polarization channels. Since with all bands the uncertainty intervals for vh and hv overlap, we only show the vh cross-polarization channel for figure clarity. When comparing the results for S-, C-, and X-band we observe an increase in backscatter over frequency, which can be explained by the increment of the surface-roughness-to-wavelength ratio. For $\mathrm{X}$ - and $\mathrm{C}$-band the vv backscatter is stronger than with hh. For S-band this also holds, although the comparison is more difficult as the $\theta$ intervals become broader. It is clear, however, that for all bands the cross response is lower than that of the co-polarization. Remarkable, at first sight, is the 


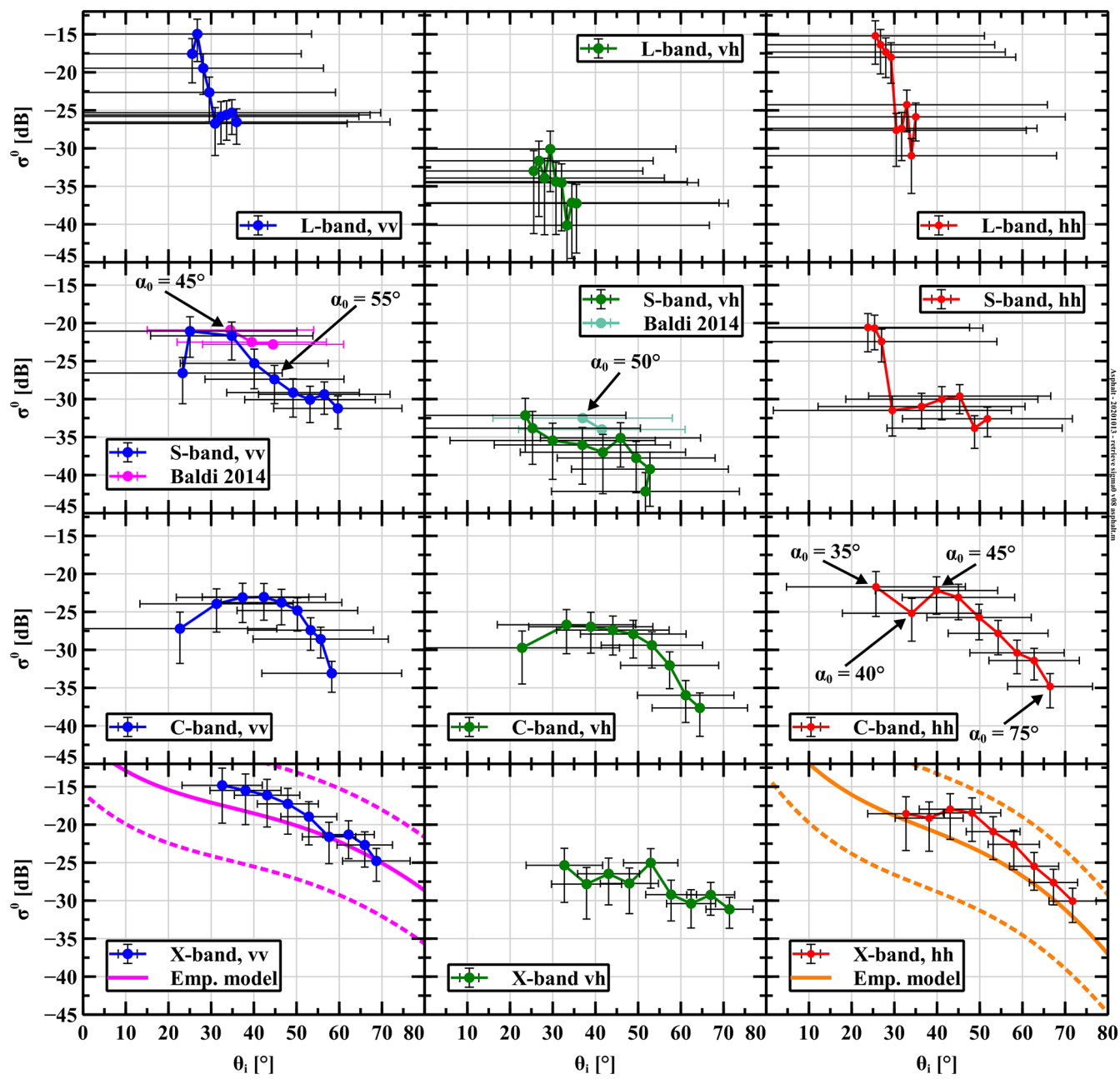

Figure F1. Measurement results of $\sigma_{p q}^{0}\left(\alpha_{0}\right)$ for all bands and polarizations together with S-band measurement results from Baldi (2014) and empirical model for X-band from Ulaby and Dobson (1989). Points represent results for different antenna boresight angles $\alpha_{0}$. Horizontal bars represent intervals for angle of incidence $\theta$, and vertical bars represent the $66 \%$ confidence interval for $\sigma^{0}$. Dotted lines between data points serve as guides. With X-band, solid and dotted curves (magenta and orange) represent the mean value and $90 \%$ confidence interval of the empirical model respectively.

fact that the retrieved $\sigma^{0}$ for L-band is higher than that of $\mathrm{S}$-band. We believe this is due to the lowest angular resolution of our system at L-band and our subsequent $\sigma^{0}$ retrieval method from the measured signal. As shown in the graphs, for L-band the backscatter from near-nadir $\theta$ angles is included in the received signal for almost all $\alpha_{0}$ angular positions. As the actual $\sigma^{0}(\theta)$, in general, shoots upward for the smaller $\theta$ angles towards the peak value at nadir, the resulting signal, and with it the retrieved $\sigma^{0}$, is high as well.

Our results are plotted together with those found in other studies. Baldi (2014) also measured asphalt backscatter for S-band. His scatterometer had a more narrow beamwidth of $10^{\circ}$, allowing for a straightforward measurement of $\sigma^{0}$ over $\theta$. He measured over $15^{\circ} \leq \theta \leq 55^{\circ}$. For a comparison to our results, we used his measured $\sigma^{0}(\theta)$ in Eq. (2) and subsequently applied our retrieval method to this simulated radar return $P^{\mathrm{RX}}$. The resulting $\sigma^{0}$ values are shown in Fig. F1. Three points for $\mathrm{vv}$ polarization and two for vh polarization could be retrieved. Because no data were presented outside the $15-55^{\circ}$ range the hh polarization response could not be simulated. In general, we consider our results to match with Baldi's satisfactorily. The differences may be attributed to fading uncertainty (low number of spatial samples) and to different surface roughness values: it seems our asphalt was smoother. However, the latter argument is speculative since neither we nor Baldi measured the surface roughness.

The only other study on L-band backscatter from asphalt we could find was that by Peake and Oliver (1971). There $\sigma^{0}$ values are reported for smooth asphalt with an estimated surface roughness of $s=0.3 \mathrm{~mm}$ for $20^{\circ} \leq \theta \leq 70^{\circ}$ for vv and $10^{\circ} \leq \theta \leq 70^{\circ}$ for hh. Because of the broad L-band $\theta$ 
ranges for our scatterometer, however, a simulation of the $\sigma^{0}$ retrieval, as with Baldi's data, would be incorrect.

For X-band with co-polarization we compare our results with the empirical model for asphalt described in Ulaby and Dobson (1989). This model is formed using measurements from multiple other studies with asphalt having various roughness values. Since our antenna beamwidths at Xband are sufficiently narrow, we can compare our results without further adjustment. No empirical model is given for asphalt at X-band with cross polarization in Ulaby and Dobson (1989). For both vv and hh polarization our retrieved $\sigma^{0}$ shows a clear overall decreasing trend over $\theta$, which is expected for a surface that is smooth compared to the wavelength. Overall, $\sigma^{0}$ for vv polarization is higher than for hh polarization, which is in accordance to the empirical model. Starting from the smaller angles, the consecutive measurement points remain at similar level. With hh polarization there appears to be even a local minimum at $40^{\circ}$, although the measurement uncertainty is relatively large there. Given that the empirical curves show a similar trend, though not as pronounced, the slow decay of $\sigma_{p p}^{0}$ over $\theta$ for $25-55^{\circ}$ can simply be a property of asphalt. Overall we find our measurements to lie within the $90 \%$ occurrence interval of the empirical model and therefore conclude that our results for asphalt are similar to those of Ulaby and Dobson (1989). We could not find studies reporting asphalt backscatter for C-band. 
Appendix G: Examples of $\sigma_{p q}^{0}$ over $\alpha_{0}$ for Maqu
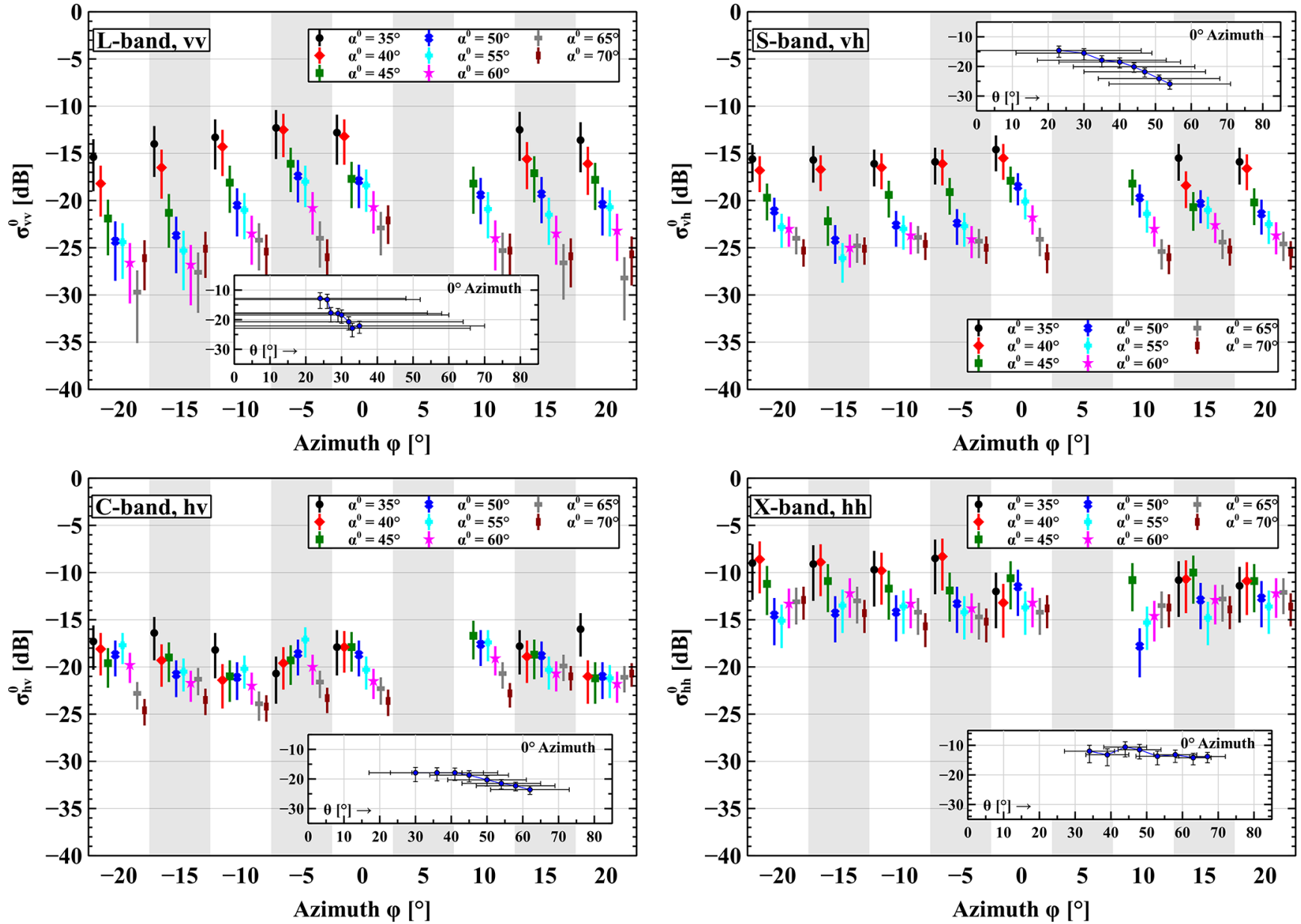

Figure G1. Measurement of $\sigma_{p q}^{0}\left(\alpha_{0}, \phi\right)$ for all bandwidths at different polarization over the Maqu site on 25 August 2017. Four main figures: for different antenna boresight azimuth angles $\phi$, the variation of $\sigma_{p q}^{0}$ over boresight elevation angles $\alpha_{0}$ is shown. The eight vertical bars represent the $66 \%$ confidence interval for $\sigma^{0}$. Intervals for incidence angles $\theta$ per measurement are not shown here for figure clarity. Insets: $\sigma_{p q}^{0}\left(\alpha_{0}\right)$ for $\phi=0^{\circ}$. Horizontal bars represent intervals of actual incidence angles $\theta$, which are identical for other $\phi$ values in the main figures. 


\section{Appendix $\mathbf{H}$}

Table H1. List of symbols.

\begin{tabular}{|c|c|c|}
\hline Symbol & Description & Unit \\
\hline$A_{\mathrm{fp}}$ & Surface area of the footprint. & $\mathrm{m}^{2}$ \\
\hline$a$ & Dimension $a$ of reference target frontal projection. & $\mathrm{m}$ \\
\hline$\alpha$ & $\begin{array}{l}\text { Angle between tower's vertical axis and the orthogonal projection of the line from antennas to a ground surface } \\
\text { segment onto the plane formed by the tower's vertical axis and the antenna boresight direction line. See also } \\
\text { Fig. B2. For antenna boresight line } \alpha=\alpha_{0} \text {. }\end{array}$ & $\circ$ \\
\hline BW & Bandwidth associated with $E_{\mathrm{e}}$ or $\sigma^{0}$ & $\mathrm{GHz}$ \\
\hline$b$ & Dimension $b$ of reference target frontal projection. & $\mathrm{m}$ \\
\hline$\beta$ & $\begin{array}{l}\text { Angle between line from antennas to a ground surface segment and projection of that same line onto the plane } \\
\text { formed by the tower's vertical axis and the antenna boresight direction line. See also Fig. B2. For antenna } \\
\text { boresight line } \beta=\beta_{0} \text {. }\end{array}$ & $\circ$ \\
\hline$c$ & Speed of light. & $\mathrm{ms}^{-1}$ \\
\hline$D$ & Antenna aperture width. & $\mathrm{m}$ \\
\hline$\Delta E_{T}$ & Temperature-induced radar return uncertainty. & $\mathrm{Vm}^{-1}$ \\
\hline$\Delta I_{N}$ & Uncertainty in $I_{N}$. & $\mathrm{W} \mathrm{m}^{-2}$ \\
\hline$\Delta K$ & Reference target measurement uncertainty. & $\mathrm{W} \mathrm{m}^{-2}$ \\
\hline$E_{\mathrm{e}}$ & Magnitude of total electric-field strength at the receive antenna, originating from the (surface) target. & $\mathrm{Vm}^{-1}$ \\
\hline$E_{\mathrm{e}}^{\mathrm{g}}$ & Same as $E_{\mathrm{e}}$; superscript $\mathrm{g}$ indicates that the time-domain filter, or gate, is applied. & $\mathrm{Vm}^{-1}$ \\
\hline$E_{\mathrm{cr}}^{\mathrm{g}}$ & $\begin{array}{l}\text { Remnant of the transmit-to-receive antenna (direct) cross coupling. This quantity is measured with antennas } \\
\text { aimed skywards; superscript } \mathrm{g} \text { indicates that the same time-domain filter, or gate, as with } E_{\mathrm{e}}^{\mathrm{g}} \text { was used. }\end{array}$ & $\mathrm{Vm}^{-1}$ \\
\hline$E_{\mathrm{b}}$ & Lowest measurable signal by scatterometer, or background value of $E_{\mathrm{e}}$. & $\mathrm{Vm}^{-1}$ \\
\hline$E_{0}^{\mathrm{g} 0}$ & $\begin{array}{l}\text { Magnitude of total electric-field strength at the receive antenna, originating from the reference target. Super- } \\
\text { script g0 indicates that the time-domain filter, or gate, is applied. }\end{array}$ & $\mathrm{Vm}^{-1}$ \\
\hline$E_{\mathrm{b} 0}^{\mathrm{g} 0}$ & Background level of $E_{0}^{\mathrm{g} 0}$. Superscript $\mathrm{g} 0$ indicates same time-domain filter, or gate, as with $E_{0}^{\mathrm{g} 0}$ is applied. & $\mathrm{Vm}^{-1}$ \\
\hline$\epsilon_{0}$ & Permittivity of vacuum (and by approximation that of air). & $\mathrm{Fm}^{-1}$ \\
\hline$\epsilon_{\text {soil }}$ & $\begin{array}{l}\text { Effective relative permittivity of a soil, which is a mixture of dry soil, water, minerals, organic material, etc. } \\
\text { Includes both real and imaginary component parts. }\end{array}$ & - \\
\hline$G$ & Antenna gain as a function of angle with respect to antenna boresight direction. Maximum value is $G_{0}$. & - \\
\hline$H_{\text {ant }}$ & Height of the antenna apertures above the ground. & $\mathrm{m}$ \\
\hline$I$ & Time-average intensity of total electric-field strength at the receive antenna, originating from the (surface) target. & $\mathrm{W} \mathrm{m}^{-2}$ \\
\hline$I_{N}$ & Measured intensity averaged over $N$ independent samples. & $\mathrm{W} \mathrm{m}^{-2}$ \\
\hline $\bar{I}$ & $\begin{array}{l}\text { The average of a large amount of independent measurements of } I \text { originating from a surface with backscattering } \\
\text { coefficient } \sigma^{0} . \bar{I} \text { is a surface property. }\end{array}$ & $\mathrm{W} \mathrm{m}^{-2}$ \\
\hline$K$ & Constant (over BW) linking $\sigma^{0}$ to $\bar{I}$. & $\mathrm{W} \mathrm{m}^{-2}$ \\
\hline$L$ & Maximum dimension of target in the context of RCS measurement. & $\mathrm{m}$ \\
\hline$M_{\mathrm{V}}$ & Spatial average volumetric top soil moisture over the Maqu site. & $\mathrm{m}^{3} \mathrm{~m}^{-3}$ \\
\hline
\end{tabular}


Table H1. Continued.

\begin{tabular}{|c|c|c|}
\hline Symbol & Description & Unit \\
\hline$m_{\mathrm{V}}$ & Volumetric soil content. & $\mathrm{m}^{3} \mathrm{~m}^{-3}$ \\
\hline$N$ & Number of independent scatterometer measurements, or samples, of a distributed target. & - \\
\hline$P_{p}^{\mathrm{RX}}$ & $\begin{array}{l}\text { Power received by radar or scatterometer. The subscript refers to the linear polarization direction (horizontal } \mathrm{h} \\
\text { or vertical v) that is measured by the antenna. }\end{array}$ & $\mathrm{W}$ \\
\hline$P_{p}^{\mathrm{TX}}$ & $\begin{array}{l}\text { Power transmitted by radar or scatterometer. The subscript refers to the linear polarization direction (horizontal } \\
\mathrm{h} \text { or vertical v) that is transmitted by the antenna. }\end{array}$ & $\mathrm{W}$ \\
\hline$P_{p}^{0}$ & $\begin{array}{l}\text { Power received by radar or scatterometer from calibration target. The subscript refers to the linear polarization } \\
\text { direction (horizontal h or vertical v) that is measured by the antenna. }\end{array}$ & $\mathrm{W}$ \\
\hline$\phi$ & Azimuth, or horizontal rotation angle of antennas. & $\circ$ \\
\hline$R$ & Distance from antennas to (area) target (segment). & $\mathrm{m}$ \\
\hline$R_{0}$ & Distance from antennas to calibration standard. & $\mathrm{m}$ \\
\hline$R_{\mathrm{ff}}$ & Distance from antennas beyond which the antenna far-field radiation region is defined. & $\mathrm{m}$ \\
\hline$R_{\mathrm{fp}}$ & Distance from antennas to centre of footprint. & $\mathrm{m}$ \\
\hline$R_{\mathrm{pw}}$ & Distance from antennas beyond which the wavefront of transmitted radiation is considered planar. & $\mathrm{m}$ \\
\hline$r_{\mathrm{sg}}$ & Start of the time-domain filter, also known as gate. & $\mathrm{m}$ \\
\hline$r_{\text {eg }}$ & End of the time-domain filter, also known as gate. & $\mathrm{m}$ \\
\hline$\sigma_{p q}$ & $\begin{array}{l}\text { Radar cross section (RCS). First subscript denotes polarization direction (horizontal h or vertical v) of the } \\
\text { scattered and second denotes that of the incident radiation. }\end{array}$ & $\mathrm{m}^{2}$ \\
\hline$\sigma_{\min }$ & Minimum detectable radar cross section (RCS) by scatterometer given a certain distance to target $R$. & $\mathrm{m}^{2}$ \\
\hline$\sigma_{p q}^{0}$ & $\begin{array}{l}\text { Backscattering coefficient. The radar cross section (RCS) associated with a distributed target over a certain } \\
\text { (physical) area. First subscript denotes polarization direction (horizontal h or vertical v) of the scattered and } \\
\text { second denotes that of the incident radiation. }\end{array}$ & $(-)$ \\
\hline$T_{\text {soil }}$ & Soil temperature. & ${ }^{\circ} \mathrm{C}$ \\
\hline$T_{\text {encl }}$ & Temperature inside VNA enclosure. & ${ }^{\circ} \mathrm{C}$ \\
\hline$\tau_{\mathrm{g}}$ & Temporal width of the time-domain filter, also known as gate. & s \\
\hline$\tau_{\mathrm{p}}$ & Temporal pulse width. & s \\
\hline$W_{\text {ant }}$ & Separation distance between the two antenna apertures. & $\mathrm{m}$ \\
\hline$w_{\mathrm{g}}$ & Spatial width of the time-domain filter, also known as gate. & $\mathrm{m}$ \\
\hline
\end{tabular}


Author contributions. JGH wrote this paper, installed and operated the scatterometer system, developed the data processing and $\sigma^{0}$ retrieval process, and performed the data analysis. RvdV advised in the experiment designs, $\sigma^{0}$ retrieval process, and paper structure. XW, ZW, and DZ handled the China customs logistics and installed and operated the scatterometer system. On a regular basis they maintained the scatterometer system and the Maqu site. CvdT advised in the $\sigma^{0}$ retrieval process. JW and ZS conceptualized the experiment design. All co-authors commented on and revised the paper.

Competing interests. The authors declare that they have no conflict of interest.

Financial support. This research has been supported in part by the ESA ELBARA-II/III loan agreement (grant no. EOPSM/2895/TC-tc), the ESA MOST Dragon IV Program (Monitoring Water and Energy Cycles at Climate Scale in the Third Pole Environment), the Netherlands Organization for Scientific Research (project no. ALW-GO/14-29), the National Natural Science Foundation of China (grant no. 41971033), and the Fundamental Research Funds for the Central Universities, CHD (grant no. 300102298307).

Review statement. This paper was edited by Birgit Heim and reviewed by two anonymous referees.

\section{References}

Axline, R. M.: Experimental and Simulated Study of Scattering from Randomly Rough Surfaces, Phd thesis, University of Kansas, Lawrence, Arkansas, USA, 1974.

Balanis, C. A.: Antenna Theory: Analysis and Design, 3rd edn., Wiley Interscience, Hoboken, NJ, USA, 2005.

Baldi, C. A.: The Design, Validation and Analysis of Surface Based S-band and D-band Polarimetric Scatterometers, Msc thesis, University of Massachusetts Amherst, MA, USA, 2014.

Bansal, R.: The Far-Field; how far is far enough?, Applied Microwave and Wireless, 11, 59-60, 1999.

Clapp, R. E.: A Theoretical and Experimental Study of Radar Ground Return, Report 1024, Massachusetts Institute of Technology, Cambridge, Massachusetts, USA, 1946.

Dente, L., Ferrazzoli, P., Su, Z., van der Velde, R., and Guerriero, L.: Combined use of Active and Passive Microwave Satellite Data to constrain a Discrete Scattering Model, Remote Sens. Environ., 155, https://doi.org/10.1016/j.rse.2014.08.031, 2014.

De Porrata-Dória i Yagüe, R., Ibars, A. B., and Martínez, L. F.: Analysis and Reduction of the Distortions induced by TimeDomain filtering techniques in Network Analyzers, IEEE T. Instrum. Meas., 47, 930-934, https://doi.org/10.1109/19.744645, 1998.

de Roo, R. D. and Ulaby, F. T.: Bistatic Specular Scattering from Rough Dielectric Surfaces, IEEE T. Antenn. Propag., 42, 220231, https://doi.org/10.1109/8.277216, 1994.
Dobson, M. C., Ulaby, F. T., Hallikainen, M. T., and El-rayes, M. A.: Microwave Dielectric Behavior of Wet Soil-Part II: Dielectric Mixing Models, IEEE T. Geosci. Remote, GE-23, 35-46, https://doi.org/10.1109/TGRS.1985.289498, 1985.

Famiglietti, J. S., Ryu, D., Berg, A. A., Rodell, M., and Jackson, T. J.: Field Observations of Soil Moisture Variability across Scales, Water Resour. Res., 44, W01423, https://doi.org/10.1029/2006wr005804, 2008.

Fung, A. K., Liu, W. Y., Chen, K. S., and Tsay, M. K.: An Improved Iem Model for Bistatic Scattering From Rough Surfaces, J. Electromagnet. Wave., 16, 689-702, https://doi.org/10.1163/156939302X01119, 2002.

Geldsetzer, T., Mead, J. B., Yackel, J. J., Scharien, R. K., and Howell, S. E. L.: Surface-Based Polarimetric C-Band Scatterometer for Field Measurements of Sea Ice, IEEE T. Geosci. Remote, 45, 3405-3416, https://doi.org/10.1109/TGRS.2007.907043, 2007.

He, Y., Guo, X., and Wilmshurst, J. F.: Comparison of Different Methods for measuring Leaf Area Index in a Mixed Grassland, Can. J. Plant Sci., 87, 803-813, https://doi.org/10.4141/CJPS07024, 2007.

Hofste, J., van der Velde, R., Wang, X., Zheng, D., Wen, J., van der Tol, C., and Su, Z.: Broadband Full Polarimetric Scatterometry for Monitoring Soil Moisture and Vegetation Properties Over a Tibetan Meadow, in: IGARSS 2018 - 2018 IEEE International Geoscience and Remote Sensing Symposium, 22-27 July 2018, Valencia, Spain, 2007-2010, Hofste2018, https://doi.org/10.1109/IGARSS.2018.8519380, 2018.

Hofste, J. G.: Matlab Source Code for retrieving Backscatter Coefficients, Data Archiving and Networked Services (DANS) [code], https://doi.org/10.17026/dans-xyf-fmkk, 2021.

Hofste, J. G., van der Velde, R., Wen, J., Wang, X., Wang, Z., Zheng, D., and Su, Z.: Long-term Ground-based Broadband Microwave Scatterometer Observations of an Alpine Meadow over the Tibetan Plateau, Data Archiving and Networked Services (DANS) [data set], https://doi.org/10.17026/dans-zfb-qegy, 2021.

Hughes, I. and Hase, T.: Measurements and their Uncertainties : a Practical Guide to Modern Error Analysis, Oxford University Press, Oxford, UK, 2010.

Hwang, J., Kwon, S., and Oh, Y.: Evaluation of Calibration Accuracy with HPS (Hongik Polarimetric Scatterometer) Aystem for Multi-Bands and Multi-Polarizations, in: IGARS 2011-2011 IEEE International Geoscience and Remote Sensing Symposium, 24-29 July 2011, Vancouver, BC, Canada, 3987-3990, https://doi.org/10.1109/IGARSS.2011.6050105, 2011.

Jersak, B. D., Dolaty, M., and Blanchard, A. J.: Time Domain Enhancement of Frequency Domain Radar CrossSection Data, Int. J. Remote Sens., 13, 2105-2119, https://doi.org/10.1080/01431169208904256, 1992.

Joseph, A. T., van der Velde, R., O’Neill, P. E., Lang, R., and Gish, T.: Effects of Corn on C- and L-band Radar Backscatter: A Correction Method for Soil Moisture Retrieval, Remote Sens. Environ., 114, 2417-2430, https://doi.org/10.1016/j.rse.2010.05.017, 2010.

Kerr, D. E. and Goldstein, H.: Radar Targets and Echoes, in: Propagation of Short Radio Waves, edited by: Kerr, D. E., McGrawHill Book Company Inc., New York, USA, 445-587, 1951.

Keysight Technologies: PNA Receiver Reduces Antenna RCS measurement Test Times, 5989-1949EN, available at: http://www. keysight.com (last access: 15 June 2021), 2017. 
Keysight Technologies: Keysight 2-port and 4-port PNA-L Network Analyzer, 9018-04407, available at: http://www.keysight. com (last access: 15 June 2021), 2018.

Keysight Technologies: Time Domain Analysis Using a Network Analyzer, 7018-01451, available at: http://www.keysight.com (last access: 15 June 2021), 2020.

Kim, Y., Jackson, T., Bindlish, R., Hong, S., Jung, G., and Lee, K.: Retrieval of Wheat Growth Parameters With Radar Vegetation Indices, IEEE Geosci. Remote S., 11, 808-812, https://doi.org/10.1109/LGRS.2013.2279255, 2014.

Kouyoumjian, R. G. and Peters, L.: Range Requirements in Radar Cross-Section Measurements, P. IEEE, 53, 920-928, https://doi.org/10.1109/PROC.1965.4070, 1965.

Kweon, S. and Oh, Y.: A Modified Water-Cloud Model With Leaf Angle Parameters for Microwave Backscattering From Agricultural Fields, IEEE T. Geosci. Remote, 53, 2802-2809, https://doi.org/10.1109/TGRS.2014.2364914, 2015

Lin, C., Rommen, B., Floury, N., Schüttemeyer, D., Davidson, M. W. J., Kern, M., Kontu, A., Lemmetyinen, J., Pulliainen, J., Wiesmann, A., Werner, C., Mätzler, C., Schneebeli, M., Proksch, M., and Nagler, T.: Active Microwave Scattering Signature of Snowpack - Continuous Multiyear SnowScat Observation Experiments, IEEE J. Sel. Top. Appl., 9, 3849-3869, https://doi.org/10.1109/JSTARS.2016.2560168, 2016.

Liu, P.-W., Judge, J., DeRoo, R. D., England, A. W., Bongiovanni, T., and Luke, A.: Dominant Backscattering Mechanisms at L-band during Dynamic Soil Moisture Conditions for Sandy Soils, Remote Sens. Environ., 178, 104-112, https://doi.org/10.1016/j.rse.2016.02.062, 2016.

Lv, S., Zeng, Y., Wen, J., Zhao, H., and Su, Z.: Estimation of Penetration Depth from Soil Effective Temperature in Microwave Radiometry, Remote Sens., 10, 519, https://doi.org/10.3390/rs10040519, 2018.

MacArthur, A., Robinson, I., Rossini, M., Davis, N., and MacDonald, K.: A Dual-Field-of-View Spectrometer System for Reflectance and Fluorescence Measurements (Piccolo Doppio) and Correction of Etaloning, in: Fifth International Workshop on Remote Sensing of Vegetation Fluorescence, 22-24 April 2014, Paris, France, European Space Agency, 2014.

Mätzler, C.: Applications of the interaction of microwaves with the natural snow cover, Remote Sensing Reviews, 2, 259-387, https://doi.org/10.1080/02757258709532086, 1987.

Miller, D. J.: The Tibetan Steppe, in: Grasslands of the world, edited by: Suttie, J. M., Reynolds, S. G., and Batello, C., Food and Agricultural Organization of the United Nations, Rome, Italy, available at: ftp://ftp.fao.org/docrep/fao/008/y8344e/y8344e00. pdf (last access: 11 June 2021), 2005.

Monakov, A. A., Vivekanandan, J., Stjernman, A. S., and Nystrom, A. K.: Spatial and frequency averaging techniques for a polarimetric scatterometer system, IEEE T. Geosci. Remote, 32, $187-$ 196, https://doi.org/10.1109/36.285201, 1994.

Nagarajan, K., Liu, P. W., De Roo, R., Judge, J., Akbar, R., Rush, P., Feagle, S., Preston, D., and Terwilleger, R.: Automated L-Band Radar System for Sensing Soil Moisture at High Temporal Resolution, IEEE Geosci. Remote S., 11, 504-508, https://doi.org/10.1109/LGRS.2013.2270453, 2014.

Nandan, V., Geldsetzer, T., Islam, T., Yackel, J. J., Gill, J. P. S., Fuller, M. C., Gunn, G., and Duguay, C.: Ku-, X- and C-band Measured and Modeled Microwave Backscatter from a Highly
Saline Snow Cover on First-year Sea Ice, Remote Sens. Environ. 187, 62-75, https://doi.org/10.1016/j.rse.2016.10.004, 2016.

Nesti, G. and Hohmann, M.: An Efficient Calibration Procedure For Polarimetric Radar Systems, in: IGARSS 1990-1990 IEEE International Geoscience and Remote Sensing Symposium, 20-24 May 1990, College Park, MD, USA, 1099-1103, https://doi.org/10.1109/IGARSS.1990.688685, 1990.

Oh, Y., Sarabandi, K., and Ulaby, F. T.: An Empirical Model and an Inversion Technique for Radar Scattering from Bare Soil Surfaces, IEEE T. Geosci. Remote, 30, 370-381, https://doi.org/10.1109/36.134086, 1992.

Peake, W. and Oliver, T. L.: The Response of Terrestrial Surfaces at Microwave Frequencies, Report 2440-7, Ohio State University, ElectroScience Laboratory, Columbus, Ohio, USA, 1971.

Peel, M. C., Finlayson, B. L., and McMahon, T. A.: Updated world map of the Köppen-Geiger climate classification, Hydrol. Earth Syst. Sci., 11, 1633-1644, https://doi.org/10.5194/hess-11-16332007, 2007.

Ross, R.: Radar Cross Section of Rectangular Flat Plates as a Function of Aspect Angle, IEEE T. Antenn. Propag., 14, 329-335, https://doi.org/10.1109/TAP.1966.1138696, 1966.

Schwank, M., Wiesmann, A., Werner, C., Mätzler, C., Weber, D., Murk, A., Völksch, I., and Wegmüller, U.: ELBARA II, an LBand Radiometer System for Soil Moisture Research, Sensors, 10, 584-612, 2010.

Schwarzbeck Mess-Elektronic OHG: Radiation Patterns BBHX 9120 LF Antenna, available at: http://www.schwarzbeck.de (last access: 11 June 2021), 2017.

Seneviratne, S. I., Corti, T., Davin, E. L., Hirschi, M., Jaeger, E. B., Lehner, I., Orlowsky, B., and Teuling, A. J.: Investigating Soil Moisture-Climate Interactions in a Changing Climate: A Review, Earth-Sci. Rev., 99, 125-161, https://doi.org/10.1016/j.earscirev.2010.02.004, 2010.

Skolnik, M.: Radar Handbook, 3rd edn., McGraw-Hill, New York, USA, 2008.

Sorensen, K. W.: A Dihedral Corner Reflector Model for Full Polarization Calibration of RCS Measurements, in: Antennas and Propagation Society Symposium 1991 Digest, 2024 June 1991, London, ON, Canada, vol. 2, 748-751, https://doi.org/10.1109/APS.1991.174947, 1991.

Stiles, J. M., Sarabandi, K., and Ulaby, F. T.: Electromagnetic Scattering from Grassland-Part II: Measurement and Modeling Results, IEEE T. Geosci. Remote, 38, 349-356, https://doi.org/10.1109/36.823930, 2000.

Su, Z., de Rosnay, P., Wen, J., Wang, L., and Zeng, Y.: Evaluation of ECMWF's Soil Moisture Analyses using Observations on the Tibetan Plateau, J. Geophys. Res.-Atmos., 118, 5304-5318, https://doi.org/10.1002/jgrd.50468, 2013.

Su, Z., Wen, J., Zeng, Y., Zhao, H., Lv, S., van der Velde, R., Zheng, D., Wang, X., Wang, Z., Schwank, M., Kerr, Y., Yueh, S., Colliander, A., Qian, H., Drusch, M., and Mecklenburg, S.: Multiyear In-Situ L-band Microwave Radiometry of Land Surface Processes on the Tibetan Plateau, Scientific Data, 7, 317, https://doi.org/10.1038/s41597-020-00657-1, 2020.

Tan, L. and Jiang, J.: Digital Signal Processing, Fundamentals and Applications, 2nd edn., Academic Press, Waltham, MA, USA, 2013. 
Ulaby, F. T. and Long, D. G.: Microwave Radar and Radiometric Remote Sensing, 4th edn., The University of Michigan Press, Ann Arbor, USA, 2017.

Ulaby, F. T., Moore, R. K., and Fung, A. K.: Microwave Remote Sensing Active and Passive Vol. II: Radar Remote Sensing and Surface Scattering and Emission Theory, Addison-Wesley Publishing Company, Reading, Massachusetts, USA, 1982.

Ulaby, F. T., Allen, C. T., and Fung, A. K.: Method for Retrieving the True Backscattering Coefficient from Measurements with a Real Antenna, IEEE T. Geosci. Remote, GE-21, 308-313, https://doi.org/10.1109/TGRS.1983.350558, 1983.

Ulaby, F. T., Haddock, T. F., and Austin, R. T.: Fluctuation Statistics of Millimeter-Wave Scattering from Distributed Targets, IEEE T. Geosci. Remote, 26, 268-281, https://doi.org/10.1109/36.3030, 1988.

Ulaby, F. W. and Dobson, M. C.: Handbook of Radar Scattering Statistics for Terrain, Artech House Inc., Norwood, MA, USA, 1989.

Vachaud, G., Passerat De Silans, A., Balabanis, P., and Vauclin, M.: Temporal Stability of Spatially Measured Soil Water Probability Density Function, Soil Sci. Soc. Am. J., 49, 822-828, https://doi.org/10.2136/sssaj1985.03615995004900040006x, 1985.

Wang, Q. and Gogineni, S.: A Numerical Procedure for Recovering Scattering Coefficients from Measurements with WideBeam Antennas, IEEE T. Geosci. Remote, 29, 778-783, https://doi.org/10.1109/36.83993, 1991.
Wang, Q., van der Velde, R., Su, Z., and Wen, J.: Aquarius Lband Scatterometer and Radiometer Observations over a Tibetan Plateau Site, Int. J. Appl. Earth Obs., 45, 165-177, https://doi.org/10.1016/j.jag.2015.06.010, 2016.

Werner, C., Wiesmann, A., Strozzi, T., Schneebeli, M., and Mätzler, C.: The Snowscat Ground-Based Polarimetric Scatterometer: Calibration and Initial Measurements from Davos Switzerland, in: 2010 IEEE International Geoscience and Remote Sensing Symposium, 25-30 July 2010, Honolulu, HI, USA, 2363-2366, Werner2010, https://doi.org/10.1109/IGARSS.2010.5649015, 2010.

Zheng, D., van der Velde, R., Su, Z., Wen, J., Wang, X., and Yang, K.: Evaluation of Noah Frozen Soil Parameterization for Application to a Tibetan Meadow Ecosystem, J. Hydrometeorol., 18, 1749-1763, https://doi.org/10.1175/jhm-d-16-0199.1, 2017a.

Zheng, D., Wang, X., Velde, R. v. d., Zeng, Y., Wen, J., Wang, Z., Schwank, M., Ferrazzoli, P., and Su, Z.: L-Band Microwave Emission of Soil Freeze-Thaw Process in the Third Pole Environment, IEEE T. Geosci. Remote, 55, 5324-5338, https://doi.org/10.1109/TGRS.2017.2705248, 2017b.

Zheng, D., Li, X., Wang, X., Wang, Z., Wen, J., van der Velde, R., Schwank, M., and Su, Z.: Sampling Depth of L-band Radiometer Measurements of Soil Moisture and Freeze-Thaw Dynamics on the Tibetan Plateau, Remote Sens. Environ., 226, 16-25, https://doi.org/10.1016/j.rse.2019.03.029, 2019. 